\title{
Crossmodal effects on texture perception and processing
}

Citation for published version (APA):

Eck, J. (2014). Crossmodal effects on texture perception and processing. [Doctoral Thesis, Maastricht University]. Maastricht University. https://doi.org/10.26481/dis.20140221je

Document status and date:

Published: 01/01/2014

DOI:

10.26481/dis.20140221je

Document Version:

Publisher's PDF, also known as Version of record

\section{Please check the document version of this publication:}

- A submitted manuscript is the version of the article upon submission and before peer-review. There can be important differences between the submitted version and the official published version of record.

People interested in the research are advised to contact the author for the final version of the publication, or visit the DOI to the publisher's website.

- The final author version and the galley proof are versions of the publication after peer review.

- The final published version features the final layout of the paper including the volume, issue and page numbers.

Link to publication

\footnotetext{
General rights rights.

- You may freely distribute the URL identifying the publication in the public portal. please follow below link for the End User Agreement:

www.umlib.nl/taverne-license

Take down policy

If you believe that this document breaches copyright please contact us at:

repository@maastrichtuniversity.nl

providing details and we will investigate your claim.
}

Copyright and moral rights for the publications made accessible in the public portal are retained by the authors and/or other copyright owners and it is a condition of accessing publications that users recognise and abide by the legal requirements associated with these

- Users may download and print one copy of any publication from the public portal for the purpose of private study or research.

- You may not further distribute the material or use it for any profit-making activity or commercial gain

If the publication is distributed under the terms of Article $25 \mathrm{fa}$ of the Dutch Copyright Act, indicated by the "Taverne" license above, 


\section{Crossmodal effects on texture perception and processing}

Judith Eck 
(C) Judith Eck, Maastricht 2014

All rights reserved. No part of this thesis may be reproduced, stored in a retrieval system, or transmitted in any form, or by any means, electronic, mechanical, photocopying, recording or otherwise, without prior written permission of the author.

Cover: Judith Eck

Production: CPI Wöhrmann Print Services B.V.

ISBN: 978-94-6203-517-1

The work presented in this thesis was funded by the European Community's Seventh Framework Programme FP7/2007-2013 under grant agreement number PITN-GA-2008-214728 and by the BrainGain Smart Mix Programme of the Dutch Ministry of Economic Affairs and the Dutch Ministry of Education, Culture and Science. 


\title{
Crossmodal effects on texture perception and processing
}

\author{
DISSERTATION
}

To obtain the degree of Doctor at Maastricht University, on the authority of Rector Magnificus, Prof. dr. L.L.G. Soete, in accordance with the decision of the Board of Deans, to be defended in public

on Friday $21^{\text {st }}$ of February 2014 at 10:00 hours

by

Judith Eck 


\section{Supervisor}

Prof. dr. R. Goebel

\section{Co-supervisor}

Dr. A.L. Kaas

\section{Assessment Committee}

Prof. dr. B. Jansma (Chair)

Prof. dr. A.M.L. Kappers (VU University Amsterdam, NL)

Prof. dr. K. Sathian (Emory University, USA)

Prof. dr. P. de Weerd

Dr. G. Valente 


\section{CONTENTS}

CHAPTER 1

General introduction

CHAPTER 2

Roughness perception of unfamiliar dot pattern textures

\section{CHAPTER 3}

71

Crossmodal interactions of haptic and visual texture information in early sensory cortex

CHAPTER 4

103

The effect of task instruction on haptic texture processing: The neural underpinning of roughness and spatial density perception

CHAPTER 5

Summary and general discussion

ACKNOWLEDGMENTS

CURRICULUM VITAE

153

PUBLICATIONS 



\section{Chapter 1}

General introduction 
Imagine standing in front of your locked apartment and searching in your crammed bag for the keys. If this happens during the day you might find them easily just by searching with your eyes. However, if this happens at night, you need to search with your hands and you have to use non-visual cues to find them, such as the rattling sound of the keys when they are touched or the shape, material and texture of the keys. This example illustrates the multisensory nature of our everyday interactions with the environment.

The attention devoted to the research field of perception is unequally distributed among the different sensory modalities. The most commonly investigated sensory modalities are, by and large, vision and audition. A quick search on Pubmed using the search terms "visual", "auditory" and "somatosensory", illustrates the fact that, although research on the somatosensory system has increased in recent years, it is still lagging behind considerably as compared to the visual and auditory systems (see Table 1). Similar results can be obtained with other search engines (e.g., Google Scholar) or by using different search terms related to the sensory mode of perception (e.g., "haptic" or "tactile").

Table 1. Number of publications provided by PubMed using different search terms.

Number of publications in recent years

\begin{tabular}{llllll} 
Search term & 2012 & 2011 & 2010 & 2009 & 2008 \\
\hline visual & 20,150 & 18,729 & 17,420 & 16,762 & 15,558 \\
auditory & 4,577 & 4,319 & 3,986 & 3,897 & 3,777 \\
somatosensory & 1,310 & 1,162 & 1,143 & 1,073 & 1,070 \\
\hline
\end{tabular}

Note. Search was performed on June 18, 2013.

Yet, of all senses, touch is the one first to develop in human fetuses (Andersen, 2008), indicating its importance for early human development. This is also visible in very young infants, who explore the external world predominantly with their mouth. Rochat (1989) reported that infants up to three months start interactions with objects mainly via oral contact, while only older infants start object exploration more often with visual inspection. Furthermore, he showed that within the first six months of life, object exploration becomes increasingly multimodal. Exploratory behaviors of infants are not limited to those executed with the mouth; in fact, from birth onwards, infants are capable of encoding shape information from simple objects with their hands (Streri and Féron, 2005). This ability is trained and improved to the point that five seconds and less are sufficient to identify familiar objects solely by touching them (Craddock and Lawson, 2008). These examples illustrate that touch is fundamental to our development. Hence, touch and the interaction between touch and other senses deserve more attention in the research field of sensory perception and processing. The present thesis aims at contributing to the 
knowledge on how we perceive information by touch and how tactile perception is influenced by visual information. To this end, this first chapter of the thesis will set the stage for the subsequent empirical work by providing a brief summary of the body of literature to date, introducing the basic concepts that are important for this line of research.

\section{TEXTURE AND ITS DEFINITION}

We can perceive different properties of our external world by touch. These include among others macrogeometric features such as the shape and size of an object and microgeometric properties such as the surface texture of an object (Roland and Mortensen, 1987). This thesis focuses on the perception and processing of the latter. Texture is commonly defined as the visual and tactile characteristics of a surface. However, texture can be characterized in several ways and although there seems to be no all-embracing definition in the literature, the following three citations provide sound descriptions of the basic essence of textures:

"The tactile world consists mainly of surfaces and objects. In every surface there are always small deviations of the surface geometry present in the form of grooves, pouts and wells that do not alter the shape of the object the surface is part of. These surface deviations are called microgeometric surface deviations." (Roland and Mortensen, 1987)

“..., it is generally understood that texture implies a homogeneous expanse of elements.” (Lederman et al., 1986)

"Texture information in particular is an inherent material characteristic of the surface of any object and provides an important cue for its perception." (Whitaker et al., 2008)

In general, the texture of a surface helps us determining out of what "stuff" it is made. Hence, texture helps us characterizing the material properties of an object. This kind of information plays a critical role in the interaction with objects in our environment. For example, we adjust the force and velocity of our hand movements depending on whether we touch a slippery piece of soap or a piece of wood.

Although we can perceive textures passively, as when an object is pressed on our skin, we usually explore textures actively by touching them. These two modes of touch rely on different sensory signals. In the passive case, the so-called tactile perception, we rely only on input from receptors in our skin (i.e., cutaneous mechanoreceptors). For active touch, the percept arises based on integrated cutaneous and kinesthetic input from mechanoreceptors in the skin, 
muscles, tendons and joints. This mode of touch is also called active haptic perception (Klatzky and Lederman, 2003). The following section provides a short introduction to the neurophysiology of tactile perception.

\section{THE NEUROPHYSIOLOGY OF TACTILE PERCEPTION}

As was already described above, a crucial component in tactile perception are the neural signals from cutaneous mechanoreceptors. These receptors respond to mechanical stimuli, such as pressure and vibration, and are responsible for the sensory transduction of mechanical energy into neural responses. There are four types of mechanoreceptors that respond to light skin deformations in the human glabrous skin: the more superficial Meissner corpuscles and Merkel cells and the more deeply embedded Pacinian corpuscles and Ruffini corpuscles. These are the cell end organs of four mechanoreceptive afferent neuron types, which are classified according to their adaptation rate and their receptive field size. The fast-adapting units respond primarily to dynamic information. One type has small and well-defined receptive fields (RA I or RA) and is connected to Meissner corpuscles, while the other one has large receptive fields (RA II or PC) and is connected to Pacinian corpuscles. RA I afferents respond to the rate of skin deformation and RA II units respond to the onset and offset of skin deformation. In contrast, slowly-adapting populations show a continuous response to sustained skin deformation. In this class, units with small receptive fields (SA I) can be distinguished from units with large receptive fields and indistinct borders (SA II). The former end in Merkel cells, while the latter likely end in Ruffini corpuscles (see Dahiya and Valle, 2013; Goodwin and Wheat, 2004; Johnson, 2001 and Vallbo, 1995).

The innervation density of the mechanoreceptors varies depending on the body location, e.g. the highest innervation density on the hand can be found at the fingertip, in particular for RA I and SA I afferents, while density decreases for the remaining part of the finger and the palm (Johansson and Vallbo, 1979). This contributes to the high tactile sensitivity of the fingertip. However, the innervation density is also different depending on the type of afferent. The density of RA I and SA I units on the fingertip was estimated to be approximately 140 and 70 units respectively, whereas RA II and SA II afferents seem to be less prominent with approximately 22 and 12 units per $\mathrm{cm}^{2}$ of the skin (Johansson and Vallbo, 1979). Table 2 summarizes the characteristics of the different mechanoafferents. 
Table 2. Properties of cutaneous mechanoreceptors.

\begin{tabular}{|c|c|c|c|c|}
\hline & RA I (or RA) & SA I & $\begin{array}{l}\text { RA II (or } \\
\text { PC) }\end{array}$ & SA II \\
\hline End organ & $\begin{array}{l}\text { Meissner } \\
\text { corpuscle }\end{array}$ & $\begin{array}{l}\text { Merkel } \\
\text { cell }\end{array}$ & $\begin{array}{l}\text { Pacinian } \\
\text { corpuscle }\end{array}$ & $\begin{array}{l}\text { Ruffini } \\
\text { corpuscle }\end{array}$ \\
\hline $\begin{array}{l}\text { Adaptation } \\
\text { rate }\end{array}$ & Fast & Slow & Fast & Slow \\
\hline $\begin{array}{l}\text { Receptive } \\
\text { field size }\end{array}$ & Small & Small & Large & Large \\
\hline $\begin{array}{l}\text { Units } / \mathrm{cm}^{2} \text { at } \\
\text { the fingertip }\end{array}$ & 140 & 70 & 22 & 12 \\
\hline $\begin{array}{l}\text { Spatial } \\
\text { acuity }\end{array}$ & Intermediate & High & Poor & Poor \\
\hline Sensitive to & $\begin{array}{l}\text { Low fre- } \\
\text { quency } \\
\text { vibration } \\
\text { (flutter), } \\
\text { dynamic skin } \\
\text { defor- } \\
\text { mation, } \\
\text { movement }\end{array}$ & $\begin{array}{l}\text { Pressure, } \\
\text { Edges, } \\
\text { corners, } \\
\text { curvature, } \\
\text { (spatial } \\
\text { structure of } \\
\text { objects and } \\
\text { surfaces) }\end{array}$ & $\begin{array}{l}\text { High } \\
\text { frequency } \\
\text { vibration }\end{array}$ & $\begin{array}{l}\text { Skin stretch, } \\
\text { motion } \\
\text { direction }\end{array}$ \\
\hline
\end{tabular}

As already indicated in Table 2, texture perception - and in particular roughness - seems to be mainly mediated by SA I afferents (Connor et al., 1990), but also by RA II units (Bensmaia and Hollins, 2005). While spatial variations in the firing rate among SA I units code roughness of textures with particle sizes that exceed 200 micrometer (macrotextures), roughness of finer textures (microtextures) seems to be mediated by the detection of vibrations which are caused by the movement of the texture across the skin. This vibratory code is represented in the amount of activity in RA II afferents (Hollins and Bensmaia, 2007). In addition to pattern roughness, Gescheider and Wright (2013) recently investigated roughness perception of the microstructure of a texture, i.e. the single surface elements. The results of this study indicated that information about the roughness of the microstructure of a texture is mediated by both SA I and the RA II channels, which operate in an opponent-like fashion. Interestingly, it was observed that structures mimicking the epidermal parallel ridges of the fingerprint result in an amplification and tuning of the tactile signal to a frequency set within the optimal range of sensitivity of RA II afferents, when these biomimetic tactile sensors are moved across a fine texture (Scheibert et al., 2009). This indicates that the mechanical filter characteristics of our skin facilitate the signal transmission to the mechanoreceptors involved in coding texture perception. 
The signal from the mechanoreceptors in the periphery is further transmitted to the brain. The mechanoreceptors are the peripheral endings of the primary sensory neurons, also called first-order neurons, which have their cell bodies in the dorsal root ganglia throughout the spine. The proximal ends of these sensory neurons enter the dorsal horn of the spinal cord. Two different pathways are formed at that point, the spinothalamic tract, carrying information about pain and temperature, and the dorsal column-medial lemniscal (DCML) pathway, carrying mainly tactile and kinesthetic information. In the DCML pathway, fibers conveying information from the lower extremities ascend in the gracile tract, while fibers from the upper limbs, the trunk and the neck ascend in the cuneate tract. The first-order axons of both tracts ascend ipsilaterally and terminate in the dorsal column nuclei of the medulla. The axons of the secondorder neurons in the medulla ascend and terminate in the ventral posterior lateral nucleus of the contralateral thalamus. The third-order neurons from the thalamus project information to the cortical neurons in the primary somatosensory cortex, located in the postcentral gyrus of the anterior parietal lobe (see Purves et al., 2004 for more information on the DCML pathway). Importantly, the topographic organization of the sensory signal, also called somatotopy, is preserved at all levels of processing up to the primary somatosensory cortex. Moreover, since Penfield and Boldrey (1937), it is known that sensitive parts of the body such as the hands and lips are allocated to more cortical space in the somatosensory cortex than less sensitive regions as the trunk. Hence, the amount of cortical space is proportional to the receptor density in the periphery. The authors illustrated that relationship in the famous sensory homunculus of the somatosensory cortex.

Based on the cytoarchitectural work of Brodmann, four different areas were distinguished in the primary somatosensory cortex: area $3 \mathrm{a}, 3 \mathrm{~b}, 1$ and 2 . There is architectonic evidence that these areas exist in the somatosensory cortex of humans (Kaas, 2004). In general, these regions are arranged from anterior to posterior, but there is a high inter-individual variability in the extent and the border of these areas (Geyer et al., 2000). Area 3a lies in the fundus of the central sulcus; area $3 \mathrm{~b}$ is located in the rostral bank of the postcentral gyrus and area 1 lies on the crown of the postcentral gyrus and extends down into the postcentral sulcus (Geyer et al., 1999). The borders and extent of area 2 in the primary somatosensory cortex seem to be also quite variable; however, this area always occupies the caudal wall of the postcentral gyrus (Grefkes et al., 2001). There is compelling evidence that all of these regions show a somatotopic organization, i.e. representational maps of the contralateral body site, which was predominantly investigated by stimulating different parts of the hand and fingers (Blankenburg et al., 2003; Gelnar et al., 1998; Kurth et al., 2000; Martuzzi et al., 2014; Sanchez-Panchuelo et al., 2012; Schweizer et al., 2008; Stringer et al., 2011).

Information from the primary somatosensory cortex (SI) is projected to the bilateral secondary somatosensory cortex (SII), located in the upper bank of 
the lateral sulcus and further to the insular cortex. Similar to SI, SII is also characterized by a somatotopic organization, which is somewhat cruder than in SI (del Gratta et al., 2002; Eickhoff et al., 2007; Ruben et al., 2001). It is currently still debated whether human SII receives only input from SI, indicating a serial processing of information (Hu et al., 2012; Inui et al., 2004; Kalberlah et al., 2013), or whether there are also direct projections from the thalamus, favoring a parallel processing (Liang et al., 2011). It is, however, undisputed that further pathways exist from SII to the amygdala and the hippocampus, which are probably involved in the formation of tactile memories (Kaas, 1993). SI also projects information to the posterior parietal cortex (PPC). Dijkerman and Haan (2007) suggested that similar to the 'ventral' and 'dorsal' pathway in the visual system (Goodale and Milner, 1992), two segregated pathways subserving perception and action can be found in the somatosensory system. The authors suggested that processing related to action occurs mainly in the PPC, whereas perception-centered processing occurs mainly in SII and the insula but also in the PPC. Furthermore, simple feature processing was assigned to the anterior parietal cortex, while higher association areas were suggested to be involved in feature combination and integration. Although the terminology used for both processing streams and the interactions of these pathways is still highly debated, several studies presented supportive data for two segregated (but interacting) somatosensory cortical pathways (e.g. de Santis et al., 2007; Reed et al., 2005; Sathian et al., 2011).

\section{THE MULTIDIMENSIONALITY OF TEXTURE PERCEPTION}

Texture perception is not a unidimensional experience; textures can be characterized and described by several different attributes such as roughness, regularity, spatial density, stickiness, slipperiness and coarseness. Several studies tackled the problem of defining the underlying perceptual dimensions of tactile textures, often using a multidimensional scaling approach (MDS) (Bergmann Tiest and Kappers, 2006; Gescheider et al., 2005; Hollins et al., 2000; Picard et al., 2003; Yoshioka et al., 2007). This method uses dissimilarity ratings of a predefined set of stimuli to illustrate the perceptual structure of the stimuli in an $\mathrm{N}$-dimensional space. A limitation of this technique is that the derived solution depends on the presented texture set, resulting in different findings across studies. Hence, the dimensionality of the derived perceptual space varies across studies, ranging from 2-3 dimensions (Hollins et al., 2000; Yoshioka et al., 2007) up to 4 dimensions (Bergmann Tiest and Kappers, 2006; Picard et al., 2003). While the number of dimensions is debated, the primary dimensions of texture perception were consistently identified as roughness, hardness and often stickiness, with additional attributes depending on the set of textures employed. 
Roughness is the texture dimension for which the largest body of literature exists. This is likely due to the fact that roughness is one of the first characteristics that come to our mind when talking about haptic texture perception. Experimentally, the importance of roughness for tactile texture discrimination was confirmed by several studies (Bergmann Tiest and Kappers, 2006; Hollins et al., 2000). Different stimuli have been used to investigate roughness, ranging from a wide set of familiar textures such as fabrics, wood and sand paper (Bergmann Tiest and Kappers, 2006; Yoshioka et al., 2007) to unfamiliar textures that can be varied parametrically such as linear gratings (Lawrence et al., 2007; Lederman, 1983; Lederman and Taylor, 1972; Kitada et al., 2005; Pruett et al., 2001; Taylor and Lederman, 1975) and dot pattern stimuli (Connor et al., 1990; Gescheider et al., 2005.; Klatzky and Lederman, 1999; Merabet et al., 2004, 2007; Smith et al., 2002; Sutu et al., 2013). While the ecological validity of textures like paper, felt and cotton is certainly higher, the experimental control of possible confounding factors such as differences in the material or in the textural pattern is very difficult or even close to impossible for natural textures. Hence, most studies used a stimulus set which was specifically designed and manufactured for the experimental investigation of roughness, varying only in predefined characteristics, e.g. the regularity or the height of dot patterns or the groove width of linear gratings. This approach allowed researchers to investigate systematically the influence of different physical parameters on perceived roughness. The physical parameters that influence roughness to varying degrees include height differences (Blake et al., 1997; Sutu et al., 2013), friction between the skin and the surface (Bergmann Tiest and Kappers, 2007) element size (Blake et al., 1997; Connor et al., 1990) and inter-element spacing (Cascio and Sathian, 2001; Connor et al., 1990; Dépeault et al., 2009; Meftah et al., 2000; Merabet et al., 2004; Sathian et al., 1989).

Another factor frequently investigated in tactile roughness perception is the mode of exploration. Since Lederman and Klatzky (1987) it is known that the tactile encoding of different object properties relies on optimal haptic exploratory procedures, i.e. hand movements. While the optimal exploratory procedure for texture perception is lateral motion, perceived roughness does not seem to differ for passive exploration (the surface is moved across the hand) and active exploration (the hand is moved across the surface) (Heller, 1989; Lederman, 1981). This led researchers to conclude that information from cutaneous mechanoreceptors but not kinesthetic input is required for roughness perception. However, Yoshioka et al. (2011) recently showed that although cutaneous input provides the necessary signal for roughness perception, kinesthetic input in the form of active or pseudo-active exploration (the hand is moved by somebody else) is required to achieve roughness constancy, e.g. across varying scanning speeds.

Although direct touch is the natural way of exploring textures, it can be informative to ask people to judge roughness indirectly via a tool (Klatzky and 
Lederman, 1999; Lederman et al., 1999; Lawrence et al., 2007). In indirect touch all information is conveyed via vibration, since no spatial deformation of the skin resulting from the direct touch of the texture is available. The difference in perceived roughness of direct and indirect touch can be used to identify the underlying neural mechanisms of roughness perception (Hollins et al., 2006). Furthermore, findings from this line of research can be useful to define effective vibratory inputs for realistic virtual texture perception (Klatzky and Lederman, 2001).

In summary, roughness perception is influenced by different physical parameters such as the inter-element spacing of textures, the size of the single elements and height differences. Surface roughness can be perceived by active and passive exploration, but roughness constancy is only achieved by providing kinesthetic input.

\section{MULTISENSORY TEXTURE PERCEPTION}

Texture perception is not only a multidimensional experience it is also multisensory. Some texture dimensions like the reflectance of a surface can be only perceived by a single sense - vision - but others are accessible to multiple senses. This multisensory information might be redundant, as in the case of spatial density of a regularly patterned texture. It is easy to differentiate small variations in the density of different embossed dot patterns by vision; nonetheless, the spatial resolution of our finger pads is also high enough to feel differences in the density. Hence, both vision and touch provide the same kind of information. The multisensory nature of texture perception is also crucial for the interaction with objects in our environment. The surface information of an object within our peripersonal space is first available to vision; therefore, it is often important that vision educates touch. Coming back to the example of grasping a slippery piece of soap, Fikes et al. (1994) observed that the precontact movement time was longer for slippery objects than for objects covered with sandpaper when subjects were asked to pick them up as fast and accurately as possible. Hence, the visual cues of the object surface provided the necessary information to adapt the behavior to the changing environmental conditions. In this example, the grasping motion was slowed down because the required precision is higher for grasping slippery than rough objects. Such a crossmodal transfer of visual and tactile texture information was also partially illustrated by Picard (2006) on a sample of car seat materials.

Although textures can be perceived by multiple senses (Bergmann Tiest and Kappers, 2007; Picard, 2006), it is generally accepted that there are systematic differences between sensory modalities. Cooke and associates asked their subjects to rate the similarity of novel objects that varied parametrically in shape and texture (Cooke et al., 2007). Participants rated these objects by vision, by touch or by vision and touch combined. Interestingly, when rated by 
vision, shape had a stronger effect on how similar objects were rated, whereas texture differences had a stronger influence on the haptic ratings. The bimodal similarity judgments fell somewhere in between these unimodal ratings, showing that both the texture and shape dimension played a role. These findings are in accordance with the modality appropriateness hypothesis (Welch and Warren, 1980), stating that whereas vision is more appropriate for perception of spatial properties, touch is better suited for the perception of texture. Klatzky et al. (1987) provided further support for this hypothesis, in a study that required subjects to sort objects that varied in shape, texture, hardness and size. The texture dimension was particularly important when subjects ordered the objects haptically, while shape and size became more important when subjects had both visual and haptic information available for sorting the objects. Hence, the macrospatial properties size and shape seem to be more salient to vision, while microspatial properties seem to be more salient to touch.

This general notion may, however, need to be refined. Indeed, it has been shown that the relative importance of the visual and tactile modality does not only depend on the stimuli, but also on the texture dimension judged (Lederman et al., 1986). A stronger emphasis on visual information was observed for tasks involving spatial density of dot pattern textures, whereas haptic information was more important for roughness-related judgments on the same stimuli. Even within the same texture dimension, differences between the contribution of the visual and haptic sense were identified. Heller (1989) reported a comparable performance of the visual and haptic modality in a smoothness detection task for coarse textures, but a superiority of touch in the same task for fine textures.

In general, the body of literature suggests that both vision and touch are effective in performing texture perception and discrimination tasks, including spatial density and roughness estimation (Heller, 1982; Jones and O'Neil, 1985; Lederman and Abbott, 1981; Lederman et al., 1986). However, the relative importance of haptic and visual information changes in multisensory settings depending on the stimuli and tasks. Despite the effectiveness of both sensory modalities in texture perception, there is no evidence that the combined use of visual and tactile texture cues results in superior task performance (Guest and Spence, 2003; Heller, 1982; Jones and O'Neil, 1985; Lederman and Abbott, 1981). For further readings on multisensory texture perception, I refer the interested reader to Klatzky and Lederman (2010) and Whitaker et al. (2008).

In the next section, I will briefly discuss the cortical representation of texture perception 


\section{CORTICAL REPRESENTATION OF TEXTURE PERCEPTION}

The anterior parietal cortex — or more specifically, the primary somatosensory cortex - plays a crucial role in the processing of input from different peripheral mechanoreceptors. Area $3 \mathrm{a}$ and 2 primarily receive input from muscle receptors, whereas area $3 \mathrm{~b}$ and 1 predominantly process information from cutaneous receptors (Kaas, 1993). There is evidence that the organization of the latter two regions is modular. Segregated maps for SA and $\mathrm{RA}$ receptors were observed in area $3 \mathrm{~b}$ and 1, indicating a modular representation of pressure, vibration and flutter stimulation (Chen et al., 2001; Friedman et al., 2004; Sur et al., 1984). Neurons in SI were also shown to respond to tactile texture discrimination of dot patterns and gratings in monkeys (Jiang et al., 1997; Tremblay et al., 1996; Sinclair and Burton, 1991a, b). However, SI is only the input region of tactile signals from the periphery; other cortical areas are also involved in haptic texture perception, as investigated in a number of neuroimaging studies on humans. Next to activation clusters in the postcentral gyrus (PoCG) - presumably SI (Deshpande et al., 2008; Sathian et al., 2011; Servos et al., 2001; Stilla and Sathian, 2008) - these studies also reported consistent texture selective activations in the parietal operculum, the insula and in early visual cortex (Deshpande et al., 2008; Sathian et al., 2011; Stilla and Sathian, 2008). The parietal operculum and posterior insula activations comprised three out of four distinct cytoarchitectonic fields described on the human parietal operculum (OP1-4) (Eickhoff et al., 2006; Eickhoff et al., 2007), namely the somatosensory OP1, OP4 and in particular OP3 (Sathian et al., 2011; Stilla and Sathian, 2008).

In line with the psychophysical literature, the dimensionality of texture perception was also taken into account when investigating its cortical mechanisms. For example, Zangaladze and colleagues (1999) reported that TMS application over the somatosensory cortex impairs tactile discrimination of gratings varying in groove width. However, this effect was not task specific, as a similar influence on task performance was observed for orientation discrimination and roughness estimation (Merabet et al., 2004; Zangaladze et al., 1999). Two studies from the same group identified a single region in the angular gyrus that was more active during spacing discrimination than during orientation discrimination (Sathian et al., 1997; Zhang et al., 2005). Interestingly, none of these studies indicated an involvement of the early visual cortex in spacing discrimination, whereas Merabet and colleagues (2004) showed exactly this in a repetitive TMS study with dot pattern textures. The involvement of the occipital cortex in tactile texture perception was further supported by Merabet et al. (2007), who showed tactile activation changes in the primary visual cortex in a roughness and spacing estimation task as compared to an active control condition. Research on the cortical representation of tactile roughness perception indicated an involvement of the parietal operculum (Burton et al., 2008; Kitada et al., 2005; Ledberg et al., 1995; Roland 
et. al., 1998; Simões-Franklin et al., 2011) and the insular cortex (Kitada et al., 2005). The human parietal operculum consists of four different cytoarchitectonic regions, but to this day, there is no conclusive evidence for a region-specific contribution to roughness perception. However, likely candidates include OP1 and OP3 (Burton et al., 2008; Simões-Franklin et al., 2011) which seem to constitute the human homologues of the secondary somatosensory cortex SII and the ventral somatosensory area VS in nonhuman primates (Eickhoff et al., 2007).

The neuroimaging literature on visual texture perception is highly consistent, indicating an involvement of brain regions along the ventral visual pathway. These include posterior occipital regions (Sathian et al., 2011; Stilla and Sathian, 2008; Cavina-Pratesi et al., 2010a, 2010b; Peuskens et al., 2004), but also parts of the anterior medial occipito-temporal cortex such as the middle collateral sulcus (Cant and Goodale, 2007, 2011; Cant and Xu, 2012; Peuskens et al., 2004, Puce et al., 1996). In a texture-segregation study, Kastner and colleagues (2000) reported that both uniform line textures and more complex line textures forming checkerboard patterns activated areas V1, V2/VP, V4, TEO, and V3A. However, comparing both texture stimuli showed significantly higher activations in V4 and TEO for the more complex textures. These findings suggest that the areas of the ventral stream contributing to visual texture perception depend on the complexity of the textures being tested. Furthermore, Hiramatsu et al. (2011) observed that while both early and higherorder visual cortex contain information about material properties, the representation along the ventral visual pathway shifts from an image-based representation to a perceptual category-related representation. Interestingly, a region around the fusiform gyrus and the collateral sulcus reflected not only visual (e.g. matte-glossy), but also non-visual material properties (e.g. smooth rough).

In summary, studies focusing on the cortical processing of tactile texture information indicate an involvement of early visual and somatosensory cortex, as well as a contribution of the parietal operculum, the insula and the posterior parietal cortex. Visual texture perception is represented in the ventral visual processing stream with an involvement of both the early occipital and the higher medial occipito-temporal cortex.

\section{METHODS USED TO INVESTIGATE TEXTURE PERCEPTION AND PROCESSING IN THIS THESIS}

The psychometric methods and tasks used to investigate texture perception and processing are manifold, e.g. texture matching and discrimination, sorting and rating tasks and magnitude estimations. Discrimination tasks are often used to calculate the just noticeable difference (JND), while measures of subjective dissimilarity on a common scale are used 
for multidimensional scaling approaches (MDS). The latter method aims at identifying the dimensions of the underlying perceptual space that are associated with the differences among stimuli. Magnitude estimation, on the other hand, is a technique standardly applied to measure the perceived intensity of a stimulus (Stevens, 1975). Subjects are asked to assign numbers in proportion to the perceived sensory magnitude produced by the physical stimulus. All methods have different advantages and disadvantages and should be chosen depending on the research question.

In the present thesis, perceived sensory qualities of the physical stimuli were assessed via ratings on a visual analogue scale (VAS). This scale consists of a horizontal line with verbal anchors at the endpoints. The participant indicates the perceived sensory intensity by placing a vertical line at the appropriate position between the endpoints. The advantage of this scale is that it is easily accessible and that no categorical responses are required from the subjects. The physical stimuli used in all experiments were embossed dot pattern textures that varied in the mean center-to-center dot spacing. These simple textures were chosen in order to be able to control the physical parameters of the stimuli and exclude confounding factors such as differences in the material and the familiarity with stimuli in the texture set.

These sensory ratings were combined with functional magnetic resonance imaging (fMRI), enabling us to relate the physical stimulus properties and the perceptual sensory qualities to the cortical processing of texture perception.

fMRI provides an indirect measure of neuronal activity through changes in the blood oxygenation which are elicited by the increased metabolic demands of active neurons. The blood oxygenation level-dependent (BOLD) contrast is the most common method used (Ogawa et al., 1990) and employs the different magnetic characteristics of oxygenated and deoxygenated hemoglobin. With increasing neuronal activity, the energy demands increase. This is compensated by the blood supply to the brain. The vascular system provides active neurons with the primary energy sources glucose and oxygen. Oxygen is transported in the red blood cells, bound to the protein hemoglobin. The magnetic characteristics of the hemoglobin molecule change depending on whether or not it is bound to oxygen. The change in the ratio of oxygenated and deoxygenated hemoglobin, due to neuronal activity, can be measured with fMRI. Immediately after stimulation, the BOLD signal begins with an initial dip due to the increased oxygen consumption at the site of neuronal activity which results in a high concentration of deoxygenated hemoglobin. The regional cerebral blood flow (rCBF) and the regional cerebral blood volume (rCBV) increase, thus resulting in an oversupply of oxygenated blood. This leads to an increase of the signal over baseline level. After 4-6 seconds, the signal reaches its peak. If the neuronal activity is prolonged over a block of time, the peak may be extended into a plateau. When the neuronal activity ceases the BOLD signal decreases to baseline and often below it, known as the post stimulus undershoot. 
While fMRI is a standard method in cognitive neuroscience today, there are specific problems that have to be taken into account when haptic stimulation is used in fMRI experiments. The statistical analyses of fMRI data assume that each voxel represents a unique location in the brain. However, if the participant moved considerably in the course of the experiment, the time course of a single voxel would represent a signal derived from different parts of the brain, violating the assumptions of fMRI data analysis. Effects of subject movement on the signal can be partially accounted for by using motion correction algorithms prior to data analysis. The effectiveness of these procedures is, however, limited by factors such as the amount of motion and the motion pattern. Especially stimulus-correlated motion is not trivial as it can mimic taskrelated signal changes and result in false-positive activations (Hajnal et al., 1994). This kind of motion pattern can be expected, for example, when subjects have to move their hand during the haptic exploration of stimuli. To overcome this problem, participants need to be carefully instructed and thoroughly trained, i.e. in a mock scanner, before starting the fMRI experiment. The training of the participants also ensures that subjects focus on the task during the fMRI experiment rather than on the correct exploration movement. When these considerations are taken into account, the combination of psychophysical methods and neuroimaging techniques can advance our understanding of how texture information is processed in the brain.

\section{RESEARCH AIM AND OUTLINE OF THIS THESIS}

This thesis addresses three research questions related to crossmodal (visuo-haptic) texture processing. Is roughness perceived differently when using touch, vision or both? (Chapter 2) How are physical texture characteristics cortically represented when they are explored by touch, vision or a combination of both? (Chapter 3) What is the effect of perceptual judgments on the cortical representation of texture characteristics? (Chapter 4)

\section{Chapter 2 - Roughness perception of unfamiliar dot pattern textures}

This chapter focuses on the influence of sensory modality and sensory familiarity with the stimulus material on roughness perception. The question is raised whether differences in the sensory familiarity influence visual, haptic or visual-haptic roughness estimation of unfamiliar textures.

In the section above on multisensory texture perception, I reported earlier findings, which indicate that both vision and touch are well suited to judge different texture dimensions. However, it was also shown that different sensory biases can be observed depending on the attended texture dimension, with haptic information receiving a stronger weight for tasks related to 
roughness perception. Importantly, most of these studies used either familiar textures or subjects were familiarized with the stimulus material in both the visual and haptic modality before the experiment started. Thus, it is unclear to what extent the similarity of the visual and the haptic modality can be explained by the sensory familiarity with the stimulus material. In order to investigate the influence of sensory familiarity on visual, haptic and visual-haptic roughness perception, a stimulus set unfamiliar to the subjects was used. The sensory familiarity was varied by controlling the first sensory experience with the stimulus material that could be either haptic, visual or a combination of both. In a series of five experiments we investigated the detailed influence of sensory modality and familiarity on roughness perception of unfamiliar dot pattern textures.

\section{Chapter 3 - Crossmodal interactions of haptic and visual texture information in early sensory cortex}

This chapter focuses primarily on the effect of physical stimulus characteristics and sensory modality on cortical texture processing. It is investigated whether crossmodal interactions of parametrically varied visual and haptic texture information can be found already in early sensory cortices when subjects are asked to explore but not to judge textures.

While there are several studies on the cortical processing of unisensory texture information, neuroimaging studies addressing multisensory or crossmodal texture perception are scarce. Evidence for an overlap of unisensory visual and haptic texture processing streams was provided by Stilla and Sathian, (2008) and Sathian et al. (2011). These authors reported both visual and haptic texture-selective activations in the posterior occipital cortex, but also in the inferior occipito-temporal cortex, i.e. the lingual gyrus. The latter region showed a significant correlation of visual and haptic activation in a texturematching task across subjects. Hence, both early and higher visual cortex represent possible candidates for a crossmodal involvement in texture processing. Purely unimodal studies report results that seem to strengthen the hypothesized involvement of the visual cortex in tactile texture perception (Hiramatsu et al., 2011; Merabet et al., 2004, 2007). However, the crossmodal interaction of visual and haptic texture information in these cortical regions has not yet been investigated. Therefore, an fMRI study was designed to investigate whether visual information influences the processing of haptic texture information in the cortex and vice versa, by providing matching and nonmatching visual-haptic input. Participants were instructed to merely explore the textures; no perceptual judgments of the textural stimuli were required. Hence, the influence of the physical texture properties on the cortical processing was the focus of the study. In accordance with earlier studies suggesting a flow of information from early sensory regions representing physical textures 
characteristics to higher-order regions representing perceptual qualities, crossmodal effects of visual and haptic input were primarily expected in early sensory cortices.

\section{Chapter 4 - The effect of task instruction on haptic texture processing: The neural underpinning of roughness and spatial density perception}

This chapter focuses on the effect of perceptual qualities of tactile textures on cortical texture processing. In an fMRI study it is investigated whether perceived tactile roughness and perceived spatial density are represented in different parts of the brain.

The findings of studies on haptic texture processing imply that different perceptual aspects of tactile textures are represented in partly segregated cortical networks (Kitada et al., 2005; Merabet, et al., 2004; Simões-Franklin et al., 2011; Zangaladze et al., 1999; Zhang et al., 2005). However, the current findings are somewhat inconclusive, as most neuroimaging studies focused either on a single texture dimension (e.g. roughness) or did not parametrically vary the perceptual level of the investigated texture dimensions (e.g. high versus low roughness). The fMRI study described in Chapter 4 revisits the question of the cortical representation of perceived spatial density and perceived roughness of tactile textures. Psychophysical methods are combined with a neuroimaging approach in order to compare a roughness estimation task to a spatial density estimation task performed on the same stimulus set and by the same participants. This approach allows us to investigate the dependency of cortical activation patterns on the attended perceptual texture characteristic. 


\section{REFERENCES}

Andersen, P.A., 2008. Haptic perception in the human foetus. In: Grunwald, M. (Ed.), Human Haptic Perception: Basics and Applications. Birkhäuser, Basel, 149154.

Bensmaia, S., Hollins, M., 2005. Pacinian representations of fine surface texture. Percept. Psychophys. 67, 842-854.

Bergmann Tiest, W.M., Kappers, A.M.L., 2007. Haptic and visual perception of roughness. Acta Psychol. (Amst) 124, 177-189.

Bergmann Tiest, W.M., Kappers, A.M.L., 2006. Analysis of haptic perception of materials by multidimensional scaling and physical measurements of roughness and compressibility. Acta Psychol. (Amst) 121, 1-20.

Blake, D.T., Hsiao, S.S., Johnson, K.O., 1997. Neural coding mechanisms in tactile pattern recognition: The relative contributions of slowly and rapidly adapting mechanoreceptors to perceived roughness. J. Neurosci.17, 7480-7489.

Blankenburg, F., Ruben, J., Meyer, R., Schwiemann, J., Villringer, A., 2003. Evidence for a rostral-to-caudal somatotopic organization in human primary somatosensory cortex with mirror-reversal in areas $3 \mathrm{~b}$ and 1. Cereb. Cortex 13, 987-993.

Burton, H., Sinclair, R.J., Wingert, J.R., Dierker, D.L., 2008. Multiple parietal operculum subdivisions in humans: Tactile activation maps. Somatosens. Mot. Res. 25, 149-162.

Cant, J.S., Goodale, M.A., 2011. Scratching beneath the surface: new insights into the functional properties of the lateral occipital area and parahippocampal place area. J. Neurosci. 31, 8248-8258.

Cant, J.S., Goodale, M.A., 2007. Attention to form or surface properties modulates different regions of human occipitotemporal cortex. Cereb. Cortex 17, 713731.

Cant, J.S., Xu, Y., 2012. Object ensemble processing in human anterior-medial ventral visual cortex. J. Neurosci. 32, 7685-7700.

Cascio, C.J., Sathian, K., 2001. Temporal cues contribute to tactile perception of roughness. J. Neurosci. 21, 5289-5296.

Cavina-Pratesi, C., Kentridge, R.W., Heywood, C.A., Milner, A.D., 2010a. Separate processing of texture and form in the ventral stream: Evidence from fMRI and visual agnosia. Cereb. Cortex 20, 433-446.

Cavina-Pratesi, C., Kentridge, R.W., Heywood, C.A., Milner, A.D., 2010b. Separate channels for processing form, texture, and color: Evidence from fMRI adaptation and visual object agnosia. Cereb. Cortex 20, 2319 - 2332.

Chen, L.M., Friedman, R.M., Ramsden, B.M., LaMotte, R.H., Roe, A.W., 2001. Finescale organization of SI (area $3 b$ ) in the squirrel monkey revealed with intrinsic optical imaging. J. Neurophysiol. 86, 3011-3029.

Connor, C.E., Hsiao, S.S., Phillips, J.R., Johnson, K.O., 1990. Tactile roughness: Neural codes that account for psychophysical magnitude estimates. J. Neurosci. 10, 3823-3836.

Cooke, T., Jäkel, F., Wallraven, C., Bülthoff, H.H., 2007. Multimodal similarity and categorization of novel, three-dimensional objects. Neuropsychologia 45, 484495.

Craddock, M., Lawson, R., 2008. Repetition priming and the haptic recognition of familiar and unfamiliar objects. Percept. Psychophys. 70, 1350-1365. 
Dahiya, R., Valle, M., 2013. Human Tactile Sensing. In: Robotic Tactile Sensing. Springer, Netherlands, pp. 19-41.

Del Gratta, C., Della Penna, S., Ferretti, A., Franciotti, R., Pizzella, V., Tartaro, A., Torquati, K., Bonomo, L., Romani, G.L., Rossini, P.M., 2002. Topographic organization of the human primary and secondary somatosensory cortices: comparison of fMRI and MEG findings. Neuroimage 17, 1373-1383.

Dépeault, A., Meftah, E.-M., Chapman, C., 2009. Tactile perception of roughness: raised-dot spacing, density and disposition. Exp. Brain Res. 197, 235-244.

Deshpande, G., Hu, X., Stilla, R., Sathian, K., 2008. Effective connectivity during haptic perception: A study using Granger causality analysis of functional magnetic resonance imaging data. Neuroimage 40, 1807-1814.

De Santis, L., Spierer, L., Clarke, S., Murray, M.M., 2007. Getting in touch: segregated somatosensory what and where pathways in humans revealed by electrical neuroimaging. Neuroimage 37, 890-903.

Dijkerman, H.C., de Haan, E.H.F., 2007. Somatosensory processes subserving perception and action. Behav. Brain Sci. 30, 189-201.

Eickhoff, S.B., Amunts, K., Mohlberg, H., Zilles, K., 2006. The human parietal operculum. II. Stereotaxic maps and correlation with functional imaging results. Cereb. Cortex 16, 268-279.

Eickhoff, S.B., Grefkes, C., Zilles, K., Fink, G.R., 2007. The somatotopic organization of cytoarchitectonic areas on the human parietal operculum. Cereb. Cortex 17, $1800-1811$.

Fikes, T.G., Klatzky, R.L., Lederman, S.J., 1994. Effects of object texture on precontact movement time in human prehension. J. Mot. Behav. 26, 325-332.

Friedman, R.M., Chen, L.M., Roe, A.W., 2004. Modality maps within primate somatosensory cortex. Proc. Natl. Acad. Sci. U.S.A. 101, 12724-12729.

Gelnar, P.A., Krauss, B.R., Szeverenyi, N.M., Apkarian, A.V., 1998. Fingertip representation in the human somatosensory cortex: an fMRI study. Neuroimage 7, 261-283.

Gescheider, G.A., Bolanowski, S.J., Greenfield, T.C., Brunette, K.E., 2005. Perception of the tactile texture of raised-dot patterns: A multidimensional analysis. Somatosens. Mot. Res. 22, 127-140.

Gescheider, G.A., Wright, J.H., 2013. Roughness perception in tactile channels: Evidence for an opponent process in the sense of touch. Somatosens. Mot. Res. 30, 120-132.

Geyer, S., Schleicher, A., Zilles, K., 1999. Areas 3a, 3b, and 1 of human primary somatosensory cortex. Neuroimage 10, 63-83.

Geyer, S., Schormann, T., Mohlberg, H., Zilles, K., 2000. Areas 3a, 3b, and 1 of human primary somatosensory cortex. Part 2. Spatial normalization to standard anatomical space. Neuroimage 11, 684-696.

Goodale, M.A., Milner, A.D., 1992. Separate visual pathways for perception and action. Trends Neurosci. 15, 20-25.

Goodwin, A.W., Wheat, H.E., 2004. Sensory signals in neural populations underlying tactile perception and manipulation. Annu. Rev. Neurosci. 27, 53-77.

Grefkes, C., Geyer, S., Schormann, T., Roland, P., Zilles, K., 2001. Human somatosensory area 2: observer-independent cytoarchitectonic mapping, interindividual variability, and population map. Neuroimage 14, 617-631.

Guest, S., Spence, C., 2003. What role does multisensory integration play in the visuotactile perception of texture? Int. J. Psychophysiol. 50, 63-80. 
Hajnal, J.V., Myers, R., Oatridge, A., Schwieso, J.E., Young, I.R., Bydder, G.M., 1994. Artifacts due to stimulus correlated motion in functional imaging of the brain. Magn. Reson. Med. 31, 283-291.

Heller, M.A., 1989. Texture perception in sighted and blind observers. Percept. Psychophy. 45, 49 - 54.

Heller, M.A., 1982. Visual and tactual texture perception: Intersensory cooperation. Percept. Psychophy. 31, 339-344.

Hiramatsu, C., Goda, N., Komatsu, H., 2011. Transformation from image-based to perceptual representation of materials along the human ventral visual pathway. Neuroimage 57, 482-494.

Hollins, M., Bensmaia, S.J., 2007. The coding of roughness. Can. J. Exp. Psychol. 61, 184-195.

Hollins, M., Bensmaia, S., Karlof, K., Young, F., 2000. Individual differences in perceptual space for tactile textures: evidence from multidimensional scaling. Percept. Psychophys. 62, 1534-1544.

Hollins, M., Lorenz, F., Harper, D., 2006. Somatosensory coding of roughness: the effect of texture adaptation in direct and indirect touch. J. Neurosci. 26, 55825588 .

Hu, L., Zhang, Z.G., Hu, Y., 2012. A time-varying source connectivity approach to reveal human somatosensory information processing. Neuroimage 62, 217-228.

Inui, K., Wang, X., Tamura, Y., Kaneoke, Y., Kakigi, R., 2004. Serial processing in the human somatosensory system. Cereb. Cortex 14, 851-857.

Jiang, W., Tremblay, F., Chapman, C.E., 1997. Neuronal encoding of texture changes in the primary and the secondary somatosensory cortical areas of monkeys during passive texture discrimination. J. Neurophysiol. 77, 1656-1662.

Johansson, R.S., Vallbo, A.B., 1979. Tactile sensibility in the human hand: relative and absolute densities of four types of mechanoreceptive units in glabrous skin. J. Physiol. 286, 283-300.

Johnson, K.O., 2001. The roles and functions of cutaneous mechanoreceptors. Curr. Opin. Neurobiol. 11, 455-461.

Jones, B., O'Neil, S., 1985. Combining vision and touch in texture perception. Percept. Psychophys. 37, 66-72.

Kaas, J.H., 2004. Evolution of somatosensory and motor cortex in primates. Anat. Rec. A Discov. Mol. Cell. Evol. Biol. 281, 1148-1156.

Kaas, J.H., 1993. The functional organization of somatosensory cortex in primates. Ann. Anat. 175, 509-518.

Kalberlah, C., Villringer, A., Pleger, B., 2013. Dynamic causal modeling suggests serial processing of tactile vibratory stimuli in the human somatosensory cortex-An fMRI study. Neuroimage 74, 164-171.

Kastner, S., de Weerd, P., Ungerleider, L.G., 2000. Texture segregation in the human visual cortex: A functional MRI study. J. Neurophysiol. 83, 2453-2457.

Kitada, R., Hashimoto, T., Kochiyama, T., Kito, T., Okada, T., Matsumura, M., Lederman, S.J., Sadato, N., 2005. Tactile estimation of the roughness of gratings yields a graded response in the human brain: an fMRI study. Neuroimage 25, 90-100.

Klatzky, R.L., Lederman, S.J., 2010. Multisensory texture perception. In: Kaiser, J., Naumer, M.J. (Eds.), Multisensory Object Perception in the Primate Brain. Springer, New York, pp. 211-230. 
Klatzky, R.L., Lederman, S.J., 2003.Touch. In: Healy, A.F., Proctor, R.W. (Eds.), Experimental Psychology, Volume 4 in Weiner, I.B. (Editor-in-Chief) Handbook of psychology. Wiley, New York, pp. 147-176.

Klatzky, R.L., Lederman, S.J., 2001. Perceiving texture through a probe. In: McLaughlin, M.L., Hespanha, J., Sukhatme, G. (Eds.), Touch in virtual environments, Prentice Hall PTR Upper Saddle River, NJ, pp. 180-193.

Klatzky, R.L., Lederman, S.J., 1999. Tactile roughness perception with a rigid link interposed between skin and surface. Percept. Psychophys. 61, 591-607.

Klatzky, R.L., Lederman, S.J., Reed, C., 1987. There's more to touch than meets the eye: The salience of object attributes for haptics with and without vision. J. Exp. Psychol. Gen. 116, 356-369.

Kurth, R., Villringer, K., Curio, G., Wolf, K.J., Krause, T., Repenthin, J., Schwiemann, J., Deuchert, M., Villringer, A., 2000. fMRI shows multiple somatotopic digit representations in human primary somatosensory cortex. Neuroreport 11, 14871491.

Lawrence, M.A., Kitada, R., Klatzky, R.L., Lederman, S.J., 2007. Haptic roughness perception of linear gratings via bare finger or rigid probe. Perception 36, 547557.

Ledberg, A., O'Sullivan, B.T., Kinomura, S., Roland, P.E., 1995. Somatosensory activations of the parietal operculum of man. A PET study. Eur. J. Neurosci. 7, 1934-1941.

Lederman, S.J., 1983. Tactual roughness perception: spatial and temporal determinants. Can. J. Psychol. 37, 498-511.

Lederman, S.J., 1981. The perception of surface roughness by active and passive touch. Bull. Psychon. Soc. 18, 253-255.

Lederman, S.J., Abbott, S.G., 1981. Texture perception: Studies of intersensory organization using a discrepancy paradigm and visual versus tactual psychophysics. J. Exp. Psychol. Hum. Percept. Perform. 7, 902-915.

Lederman, S.J., Klatzky, R.L., 1987. Hand movements: a window into haptic object recognition. Cogn. Psychol. 19, 342-368.

Lederman S.J., Klatzky, R.L., Hamilton, C.L., Ramsay, G.I., 1999. Perceiving roughness via a rigid probe: Psychophysical effects of exploration speed and mode of touch. Electr. J. Hapt. Res. 1, 1-20.

Lederman, S.J., Taylor, M.M., 1972. Fingertip force, surface geometry, and the perception of roughness by active touch. Percept. Psychophys. 12, 401-408.

Lederman, S.J., Thorne, G., Jones, B., 1986. Perception of texture by vision and touch: Multidimensionality and intersensory integration. J. Exp. Psychol. Hum. Percept. Perform. 12, 169-180.

Liang, M., Mouraux, A., Iannetti, G.D., 2011. Parallel processing of nociceptive and non-nociceptive somatosensory information in the human primary and secondary somatosensory cortices: evidence from dynamic causal modeling of functional magnetic resonance imaging data. J. Neurosci. 31, 8976-8985.

Martuzzi, R., van der Zwaag, W., Farthouat, J., Gruetter, R., Blanke, O., 2014. Human finger somatotopy in areas $3 \mathrm{~b}, 1$, and 2: A $7 \mathrm{~T}$ fMRI study using a natural stimulus. Hum Brain. Mapp. 35, 213-226.

Meftah, E.-M., Belingard, L., Chapman, C.E., 2000. Relative effects of the spatial and temporal characteristics of scanned surfaces on human perception of tactile roughness using passive touch. Exp. Brain Res. 132, 351-361. 
Merabet, L.B., Swisher, J.D., McMains, S.A., Halko, M.A., Amedi, A., Pascual-Leone, A., Somers, D.C., 2007. Combined activation and deactivation of visual cortex during tactile sensory processing. J. Neurophysiol. 97, 1633-1641.

Merabet, L., Thut, G., Murray, B., Andrews, J., Hsiao, S., Pascual-Leone, A., 2004. Feeling by sight or seeing by touch? Neuron 42, 173-179.

Ogawa, S., Lee, T.M., Kay, A.R., Tank, D.W., 1990. Brain magnetic resonance imaging with contrast dependent on blood oxygenation. Proc. Natl. Acad. Sci. U.S.A. 87, 9868-9872.

Penfield, W., Boldrey, E., 1937. Somatic motor and sensory representation in the cerebral cortex of man as studied by electrical stimulation. Brain 60, 389-443.

Peuskens, H., Claeys, K.G., Todd, J.T., Norman, J.F., van Hecke, P., Orban, G.A., 2004. Attention to 3-D Shape, 3-D Motion, and Texture in 3-D Structure from Motion Displays. J. Cogn. Neurosci. 16, 665-682.

Picard, D., 2006. Partial perceptual equivalence between vision and touch for texture information. Acta Psychol. (Amst) 121, 227-248.

Picard, D., Dacremont, C., Valentin, D., Giboreau, A., 2003. Perceptual dimensions of tactile textures. Acta Psychol. (Amst) 114, 165-184.

Pruett, J.R., Jr., Sinclair, R.J., Burton, H., 2001. Neural correlates for roughness choice in monkey second somatosensory cortex (SII). J. Neurophysiol. 86, 2069-2080.

Puce, A., Allison, T., Asgari, M., Gore, J.C., McCarthy, G., 1996. Differential sensitivity of human visual cortex to faces, letterstrings, and textures: A functional magnetic resonance imaging study. J. Neurosci. 16, 5205-5215.

Purves, D., Augustine, G.J., Fitzpatrick, D., Hall, W.C., La Mantia, A.-S., Mc Namara, J.O., Williams, S.M. (Eds.), 2004. Neuroscience. $3^{\text {rd }}$ edition. Sinauer Associates, Sunderland.

Reed, C.L., Klatzky, R.L., Halgren, E., 2005. What vs. where in touch: an fMRI study. Neuroimage 25, 718-726.

Rochat, P., 1989. Object manipulation and exploration in 2- to 5-month-old infants. Dev. Psychol. 25, 871-884.

Roland, P.E., Mortensen, E., 1987. Somatosensory detection of microgeometry, macrogeometry and kinesthesia in man. Brain Res. Rev. 12, 1-42.

Roland, P.E., O’Sullivan, B., Kawashima, R., 1998. Shape and roughness activate different somatosensory areas in the human brain. Proc. Natl. Acad. Sci. U.S.A. 95, 3295-3300.

Ruben, J., Schwiemann, J., Deuchert, M., Meyer, R., Krause, T., Curio, G., Villringer, K., Kurth, R., Villringer, A., 2001. Somatotopic organization of human secondary somatosensory cortex. Cereb. Cortex 11, 463-473.

Sanchez-Panchuelo, R.M., Besle, J., Beckett, A., Bowtell, R., Schluppeck, D., Francis, S., 2012. Within-digit functional parcellation of Brodmann areas of the human primary somatosensory cortex using functional magnetic resonance imaging at 7 tesla. J. Neurosci. 32, 15815-15822.

Sathian, K., Goodwin, A.W., John, K.T., Darian-Smith, I., 1989. Perceived roughness of a grating: correlation with responses of mechanoreceptive afferents innervating the monkey's fingerpad. J. Neurosci. 9, 1273-1279.

Sathian, K., Lacey, S., Stilla, R., Gibson, G.O., Deshpande, G., Hu, X., LaConte, S., Glielmi, C., 2011. Dual pathways for haptic and visual perception of spatial and texture information. Neuroimage 57, 462-475.

Sathian, K., Zangaladze, A., Hoffman, J.M., Grafton, S.T., 1997. Feeling with the mind's eye. Neuroreport 8, 3877-3881. 
Scheibert, J., Leurent, S., Prevost, A., Debregeas, G., 2009. The role of fingerprints in the coding of tactile information probed with a biomimetic sensor. Science 323, 1503-1506.

Schweizer, R., Voit, D., Frahm, J., 2008. Finger representations in human primary somatosensory cortex as revealed by high-resolution functional MRI of tactile stimulation. Neuroimage 42, 28-35.

Servos, P., Lederman, S., Wilson, D., Gati, J., 2001. fMRI-derived cortical maps for haptic shape, texture, and hardness. Cogn. Brain. Res. 12, 307-313.

Simões-Franklin, C., Whitaker, T.A., Newell, F.N., 2011. Active and passive touch differentially activate somatosensory cortex in texture perception. Hum. Brain Mapp. 32, 1067-1080.

Sinclair, R.J., Burton, H., 1991a. Neuronal activity in the primary somatosensory cortex in monkeys (Macaca mulatta) during active touch of textured surface gratings: responses to groove width, applied force, and velocity of motion. J. Neurophysiol. 66, 153-169.

Sinclair, R.J., Burton, H., 1991b. Tactile discrimination of gratings: psychophysical and neural correlates in human and monkey. Somatosens. Mot. Res 8, 241-248.

Smith, A.M., Chapman, C.E., Deslandes, M., Langlais, J.S., Thibodeau, M.P., 2002. Role of friction and tangential force variation in the subjective scaling of tactile roughness. Exp. Brain Res. 144, 211-223.

Stevens, S.S., 1975. Psychophysics: Introduction to its perceptual, neural, and social prospects. John Wiley, New York.

Stilla, R., Sathian, K., 2008. Selective visuo-haptic processing of shape and texture. Hum. Brain. Mapp. 29, 1123-1138.

Streri, A., Féron, J., 2005. The development of haptic abilities in very young infants: From perception to cognition. Infant Behav. Dev. 28, 290-304.

Stringer, E.A., Chen, L.M., Friedman, R.M., Gatenby, C., Gore, J.C., 2011. Differentiation of somatosensory cortices by high-resolution fMRI at $7 \mathrm{~T}$. Neuroimage 54, 1012-1020.

Sur, M., Wall, J.T., Kaas, J.H., 1984. Modular distribution of neurons with slowly adapting and rapidly adapting responses in area $3 \mathrm{~b}$ of somatosensory cortex in monkeys. J. Neurophysiol. 51, 724-744.

Sutu, A., Meftah, E.-M., Chapman, C.E., 2013. Physical determinants of the shape of the psychophysical curve relating tactile roughness to raised-dot spacing: implications for neuronal coding of roughness. J. Neurophysiol. 109, 14031415.

Taylor, M.M., Lederman, S.J., 1975. Tactile roughness of grooved surfaces: A model and the effect of friction. Percept. Psychophys. 17, 23-36.

Tremblay, F., Ageranioti-Belanger, S.A., Chapman, C.E., 1996. Cortical mechanisms underlying tactile discrimination in the monkey. I. Role of primary somatosensory cortex in passive texture discrimination. J. Neurophysiol. 76, 3382-3403.

Vallbo, A.B., 1995. Single-afferent neurons and somatic sensation in humans. In: Gazzaniga, M.S. (Ed.), The Cognitive Neurosciences. MIT Press, Cambridge, pp. 237-252.

Welch, R.B., Warren, D.H., 1980. Immediate perceptual response to intersensory discrepancy. Psychol. Bull. 88, 638-667. 
Whitaker, T.A., Simões-Franklin, C., Newell, F.N., 2008. Vision and touch: Independent or integrated systems for the perception of texture? Brain Res. 1242, 59-72.

Yoshioka, T., Bensmaia, S.J., Craig, J.C., Hsiao, S.S., 2007. Texture perception through direct and indirect touch: An analysis of perceptual space for tactile textures in two modes of exploration. Somatosens. Mot. Res. 24, 53-70.

Yoshioka, T., Craig, J.C., Beck, G.C., Hsiao, S.S., 2011. Perceptual constancy of texture roughness in the tactile system. J. Neurosci. 31, 17603-17611.

Zangaladze, A., Epstein, C.M., Grafton, S.T., Sathian, K., 1999. Involvement of visual cortex in tactile discrimination of orientation. Nature 401, 587-590.

Zhang, M., Mariola, E., Stilla, R., Stoesz, M., Mao, H., Hu, X., Sathian, K., 2005. Tactile discrimination of grating orientation: fMRI activation patterns. Hum. Brain Mapp. 25, 370-377. 



\section{Chapter 2}

\section{Roughness perception of unfamiliar dot pattern textures}

Based on: Eck, J., Kaas, A.L., Mulders, J.L., Goebel, R., 2013. Roughness perception of unfamiliar dot pattern textures. Acta Psychol. (Amst) 143, 20-34. 


\section{ABSTRACT}

Both vision and touch yield comparable results in terms of roughness estimation of familiar textures as was shown in earlier studies. To our knowledge, no research has been conducted on the effect of sensory familiarity with the stimulus material on roughness estimation of unfamiliar textures.

The influence of sensory modality and familiarity on roughness perception of dot pattern textures was investigated in a series of five experiments. Participants estimated the roughness of textures varying in mean center-to-center dot spacing in experimental conditions providing visual, haptic and visual-haptic combined information.

The findings indicate that roughness perception of unfamiliar dot pattern textures is well described by a bi-exponential function of inter-dot spacing, regardless of the sensory modality used. However, sensory modality appears to affect the maximum of the psychophysical roughness function, with visually perceived roughness peaking for a smaller inter-dot spacing than haptic roughness. We propose that this might be due to the better spatial acuity of the visual modality. Individuals appeared to use different visual roughness estimation strategies depending on their first sensory experience (visual vs. haptic) with the stimulus material, primarily in an experimental context which required the combination of visual and haptic information in a single bimodal roughness estimate. Furthermore, the similarity of findings in experimental settings using real and virtual visual textures indicates the suitability of the experimental setup for neuroimaging studies, creating a more direct link between behavioral and neuroimaging results. 


\section{INTRODUCTION}

Texture as a surface property plays a crucial role in object identification and recognition (Lacey et al., 2010) as well as in interaction with objects in our environment. We can tell peaches and nectarines apart not because of the color or the geometry but because of their different surface textures. Texture perception has been predominantly investigated in the haptic domain, as the haptic sense appears to be dominant in perceiving material properties (Klatzky et al., 1987). However, our visual and auditory senses also process texture information. Roughness, in particular, can be distinguished not only by touch but also by the different looks of rough and smooth textures (Bergmann Tiest and Kappers, 2007) and by the different sounds that are generated when touching those surfaces (Guest et al., 2002).

It is important to differentiate roughness as a perceptual quality from the physical characteristics that evoke it. In the present study, we will refer to perceived roughness to stress the subjective character. Roughness as a physical concept was previously defined as a measure of height differences combined with spatial properties of the surface (Bergmann Tiest and Kappers, 2006). At the level of sensory neurons, haptic roughness perception is mainly mediated by a channel sensitive to vibratory information and a channel sensitive to spatial variance, as already hypothesized by Katz in 1925 (Katz, 1989). Evidence for this so-called duplex model of (tactual) roughness perception was found in a number of experiments by Hollins and colleagues (Bensmaia and Hollins, 2005; Hollins and Risner, 2000). The authors reported that roughness perception was crucially dependent on vibrotactile cues for particle sizes below $100 \mu \mathrm{m}$. The spatial pressure distribution was more important for coarser textures, for which the roughness percept was highly correlated with variation in firing rate of SA1 afferents (Blake et al., 1997; Connor et al., 1990).

Perceived roughness is influenced by physical and physiological as well as context parameters (see Bergmann Tiest, 2010). The literature is rich in studies investigating these influences, though primarily in the haptic domain. A recent review by Bergmann Tiest (2010) concluded that tactual roughness perception is associated with physical surface properties such as height difference, friction, spatial period and dot spacing. Both natural familiar textures, like fabrics, wood, foams and glass (Bergmann Tiest and Kappers, 2006; Yoshioka et al., 2007) and unfamiliar textures like linear gratings (Cascio and Sathian, 2001; Lawrence et al., 2007) and dot pattern stimuli (Dépeault et al., 2009; Kahrimanovic et al., 2009; Klatzky and Lederman, 1999; Lederman et al., 1999) have been used in a wide range of experiments investigating roughness perception. The current study employed dot pattern stimuli, because they have the advantage that one dimension can be varied parametrically while keeping all other dimensions constant. An additional advantage of (isotropic) dot patterns is that the evoked percept is independent of the haptic exploration direction. In previous studies, increasing dot diameter resulted in a decreased 
haptic roughness percept (Blake et al., 1997; Connor et al., 1990), whereas dot height influenced perceived roughness only for dot patterns with rather small dot diameters (e.g., $0.25-1.60 \mathrm{~mm}$ ) (Blake et al., 1997). (Ir)regularity of the pattern (Dépeault et al., 2009) as well as scanning speed of the textures (Meftah et al., 2000) did not affect roughness perception with the bare finger. The main factor contributing to roughness perception of these dot patterns appeared to be the mean inter-element spacing of the textures, with an increased roughness percept for increasing inter-dot spacing (Connor et al., 1990; Dépeault et al., 2009; Gescheider et al., 2005; Lederman et al., 1986; Meftah et al., 2000; Merabet et al., 2004, 2007). However, it is still a matter of debate whether this psychophysical function is more or less linear (Dépeault et al., 2009; Meftah et al., 2000) or rather represented by an inverted U-shape peaking at about $3 \mathrm{~mm}$ inter-dot spacing (Connor et al., 1990; Gescheider et al., 2005; Merabet et al., 2004, 2007). The curves depicted in these latter studies suggest that the perceived roughness function has a steep positive slope for inter-element spacings below $3 \mathrm{~mm}$ and a shallower negative slope above $3 \mathrm{~mm}$, indicating that a simple symmetric function with a negative quadratic term would not be accurate enough for modeling haptic roughness perception.

Although the factors influencing tactile roughness perception are relatively well-studied, far less is known about the way the shape of the psychophysical roughness function is influenced by the interaction and combination of visual and haptic information. Lederman and Abbott (1981) reported comparable results in a visual and haptic roughness estimation experiment using abrasive paper. The way in which humans integrate visual and haptic texture information was shown to be well described by a weighted average model of both sources of sensory information in studies using matching and discrimination tasks as well as discrepancy paradigms (Jones and O'Neil, 1985; Lederman and Abbott, 1981; Lederman et al., 1986). These studies showed that both visual and haptic information are taken into account for bimodal texture perception, but that the relative bias between visual and haptic information can be altered depending on the texture dimension judged by the observer (e.g. roughness or spatial density). Moreover, orderings of natural stimuli based on physical characteristics (height variability) showed a similar correspondence with orderings based on visual or haptic perception (Bergmann Tiest and Kappers, 2007). This indicates that both modalities are well suited for texture perception.

It is unclear to what extent the similarity of the two modalities can be explained by the subjects' level of familiarity with the stimulus material and the extensive training in its handling. If the visual stimulus is associated with a haptic memory, e.g., of the level of skin displacement and heat transfer into the material, the roughness percept arising for visual stimuli could be essentially haptic in nature. This would mean that different visual psychophysical curves could be expected for unfamiliar textures, e.g. textures used in a virtual reality setting providing only visual feedback. Alternatively, the visual sense could 
also be capable of directly judging roughness, for instance, by assessing the spatial density of a dot pattern texture. In that case, the system would need to resolve the different spatial resolutions of the haptic and the visual modality in case the roughness information from these two modalities is to be integrated. Studies investigating tactile acuity report threshold sizes between 1.21 and 3.50 $\mathrm{mm}$, with an age-related decline in tactile acuity of close to $1 \%$ a year, as summarized by Legge et al. (2008). Average human visual acuity is better and corresponds to an image of about $0.005 \mathrm{~mm}$ on the retina (Sekuler and Blake, 2001). A person with normal visual acuity (defined as the ability to resolve lines separated by one arcmin) would be able to resolve a spatial period of about 0.4 $\mathrm{mm}$ at a viewing distance of $75 \mathrm{~cm}$.

The present study aimed to characterize visually, haptically and visuohaptically perceived roughness as a function of inter-dot spacing in a series of five experiments using unfamiliar dot patterns. The unfamiliarity with the stimulus material ensured that pre-existing differences in the haptic and visual experience with the stimulus material were negligible. In the first experiment we tested whether surface roughness is judged differently when subjects are provided with only visual or only haptic information, and how visual and haptic information are combined when both visual and haptic information are available. In addition, we studied whether roughness estimates are influenced by the modality in which the stimuli are presented first, i.e. by sensory familiarity with the stimulus material.

The visual stimuli in experiment 1 were computer-rendered images of models of the real textures to enable future use of this set-up in a magnetic resonance (MR) scanner with restricted viewing of the hand. In order to rule out the possibility that our findings resulted from unintended differences between real and virtual stimuli, we repeated the experiment with a direct visual presentation of the same textures used for the haptic modality condition. Similar findings in the first and the second experiment would indicate the suitability of the experimental design for neuroimaging studies, and would establish a more direct link between behavioral and neuroimaging results. In a third experiment we investigated whether visual and haptic roughness judgments are automatically combined or whether this changes in a unimodal task context that does not explicitly require the combination of visual and haptic information. In the fourth experiment we investigated whether the inherent difference in spatial resolution between vision and touch affects unimodal roughness judgments by extrapolating the stimulus set to include visual textures with finer spatial detail, i.e. close to and below the visual spatial acuity threshold. When stimuli are presented on a monitor without any scale reference (e.g. an image of the hand) as the case in experiment 4 , it is conceivable that a difference in estimated size of the visual textures with respect to the more natural haptic presentation condition might influence visual roughness perception. An over- or underestimation of the texture size could in turn result in a miscalculation of the size of the texture elements. In a study by Bergmann Tiest and Kappers (2007) 
subjects reported that next to the number and depth of indentations also the size of them influenced their visual roughness decisions of everyday materials. The fifth experiment was designed to account for the possibility that a difference in estimated size might have influenced visual roughness perception by presenting different scale references (i.e., different hand sizes) next to the stimuli for size comparisons.

We expected that either a linear or an inverted U-shape model would be suitable to capture the characteristics of the relationship of inter-dot spacing and roughness perception, in accordance with the two dominant findings in the literature mentioned above. For modeling the inverted U-shape function we used a bi-exponential model, which is characterized by a negative exponential with a steep slope followed by an exponential decrease with a shallower slope. An example of the asymmetric shape of such a bi-exponential function is presented in Figure 1. This shape matches the asymmetric inverted U-shape profile of the data presented in previous studies (Connor et al., 1990; Gescheider et al., 2005; Merabet et al., 2004, 2007). Other studies addressed the problem of modeling non-normal distributions by applying log-log transformations to the data (Klatzky and Lederman, 1999; Lederman et al., 1999), which has the effect of normalizing positively skewed distributions and has the added benefit of variance stabilization. However, this approach would make it difficult to attain one of the main goals of the present study: a direct comparison of a linear model (in untransformed space) and a model capturing the inverted U-shape (in transformed space). In order to enable this comparison, we chose to use a relatively simple asymmetric function, the bi-exponential function, which can be used to fit the roughness as perceived by the subjects in the non-transformed space.

We compared the linear and the bi-exponential function for all subjects individually and separately for the visual, haptic and the bimodal condition. When taking the roughness judgment results for familiar textures (abrasive paper) by Lederman and Abbott (1981) into account, we would expect to find no significant difference between roughness perceived by touch and vision.

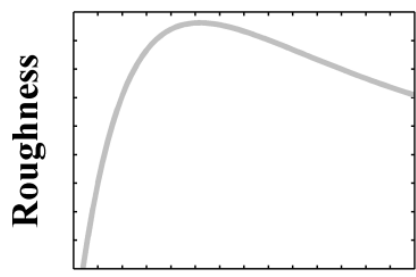

Dot Spacing

Figure 1. Model of the bi-exponential function. 
However, we cannot be certain whether those results can be generalized to the kind of unfamiliar textures we used, because it was demonstrated that factors like task and stimulus material influence the effect of sensory modality on texture perception (Guest and Spence, 2003a; Heller, 1989; Lederman et al., 1986). In the case that magnitude estimates are discrepant between the visual and haptic condition, we would expect to find a stronger emphasis on haptic than on visual information for bimodal roughness judgments in accordance with the results of a crossmodal discrepancy experiment conducted by Lederman et al. (1986).

\section{GENERAL METHOD}

\section{Participants}

The study was approved by the local ethics committee. All participants were graduate and undergraduate students recruited via an advertisement at Maastricht University. Subjects gave written informed consent, were naïve to the hypotheses and received course credit or monetary compensation for the participation in the experiment. Participants had normal or corrected-to-normal vision and subjects with callused fingertips or a history of injury to the dominant hand were excluded.

\section{Stimuli and apparatus}

The stimuli consisted of $50 \times 50 \mathrm{~mm}$ plastic plates with different embossed dot patterns. The dots were $0.6 \mathrm{~mm}$ in elevation and $0.8 \mathrm{~mm}$ in diameter with smoothed edges to prevent any harm to the skin. The dots on the plates were arranged non-periodically. The only characteristic varying between the surfaces was the mean center-to-center dot spacing. The algorithm used to produce a different inter-element spacing was derived from a study by Lederman et al. (1986). First, a regular dot-matrix was produced with a specified inter-dot spacing.

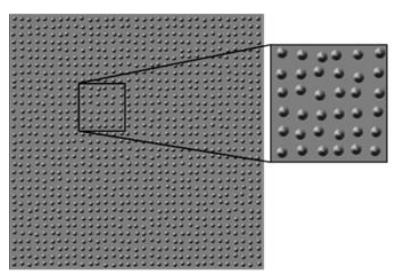

(a) $1.50 \mathrm{~mm}$

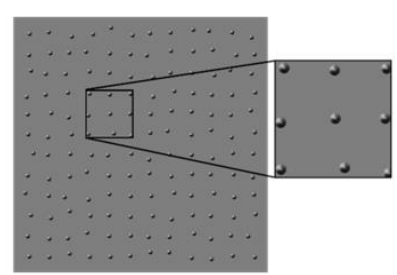

(b) $4.00 \mathrm{~mm}$

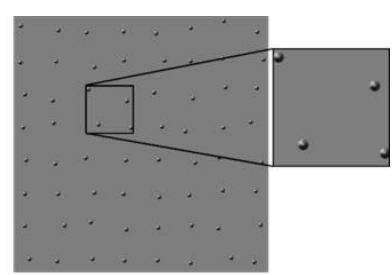

(c) $6.50 \mathrm{~mm}$

Figure 2. Downscaled examples of the surfaces presented to the subjects. 
Afterwards, the dots were randomly jittered with a particular radius to create a non-regular pattern while preserving the average inter-dot spacing. This jitter radius ranged from $0.045 \mathrm{~mm}$ for dot patterns with the smallest spacing to $2.125 \mathrm{~mm}$ for textures with the biggest spacing. For each dot matrix a 3D wireframe model was created and computer-rendered in AutoCAD 2010 (Autodesk Inc., San Rafael, CA, USA) in order to create a set of matching visual stimuli. Two distant light sources following the direction of the viewpoint provided the lighting of each stimulus in such a way that all faces of the model were illuminated (see Figure 2).

For the haptic stimulus presentation, the textures were arranged around the outside edge of a rotating circular wooden plate with a diameter of $52 \mathrm{~cm}$ and were covered with a second wooden plate. A rectangular cut-out in the cover plate enabled subjects to touch only one stimulus at a time. In addition, an opaque cloth curtain hid the stimulus from view. In all but one experiment (i.e., the second one) were the corresponding visual stimuli presented on a Full HD monitor centered in front of the subjects (see Figure 3). The choice to present the textures on a monitor instead of direct visual exploration of the real stimuli was made since a similar study was planned inside a magnetic resonance imaging (MRI) scanner with restricted viewing conditions; presenting the textures on a monitor allows the comparison of data obtained inside and outside the scanner. Furthermore, it was previously shown that visual roughness estimation changes with variations in illumination (Ho et al., 2006) and viewpoint (Ho et al., 2007). The presentation of the textures on a screen enabled us to control those factors for all subjects and trials. A single lighting model and the frontoparallel viewpoint for all dot patterns provided constant depth information across all visual stimuli. The distance between the eyes of the subject and the monitor was kept constant between 70 and $80 \mathrm{~cm}$ and the stimuli subtended $5^{\circ}$ of visual angle.

\section{Procedure}

In experiments $1-3$, subjects sat at a table on which the stimulus presentation plate was placed along with a monitor behind it. In experiments 4 and 5, the setup was the same excluding the stimulus presentation plate, which was not needed in a mere visual task. Furthermore, a chin-rest was used in experiments 4 and 5 to provide an objective control for the distance between the subject's eyes and the monitor. Subjects were informed that they would be presented with one texture at a time and were asked to evaluate the roughness of this texture. 


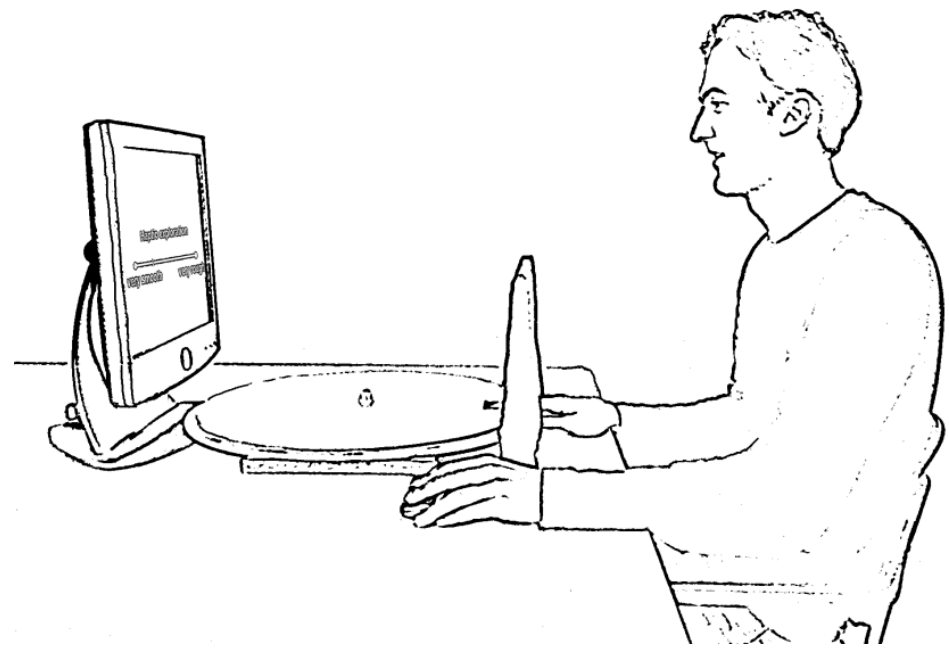

Figure 3. Schematic representation of the experimental setup.

No specific roughness definition was provided. The order of the stimulus presentation was randomized. Prior to the start of each condition, subjects were familiarized with the task and the stimuli in practice trials. During practice, example textures with increasing inter-dot spacing were presented in ascending order. This procedure was used to enable subjects to choose an appropriate rating scale for each sensory modality (Dépeault et al., 2009; Meftah et al., 2000). In all experiments in which the visual stimuli were presented on a monitor, participants were instructed to focus on the screen at all times throughout the experiment. On every trial the exploration period was preceded by the presentation of a fixation cross on the screen. This time was used by the experimenter to rotate the presentation table to the correct stimulus. In the visual-haptic condition participants were instructed to inspect the haptic and the identical visual texture simultaneously. Since it was previously demonstrated that there is only a negligible accuracy difference between the preferred and non-preferred hand (Heller, 1982; Jones and O'Neil, 1985), the dominant hand was used exclusively for all trials. Subjects were permitted to scan the textures with their index, middle and ring fingers without any time constraints, but were restricted to exploration movements toward and away from the body. In addition, they were asked to keep the same contact force for all trials. The kind of haptic exploration was predetermined to minimize differences in ratings due to different exploration strategies between conditions and subjects. After subjects finished the examination of the stimulus they used a visual analogue scale (VAS) presented on the bottom of the screen to rate the roughness of the texture from very smooth to very rough by moving a red slider via the mouse with their non-dominant hand. The experimental procedure is summarized in Figure 4. 


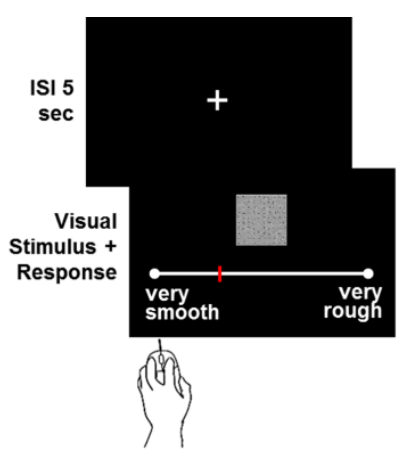

(a) Visual condition

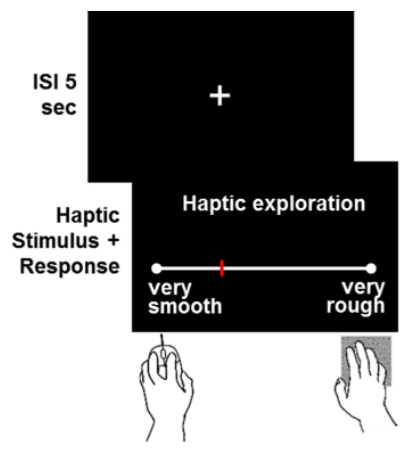

(b) Haptic condition

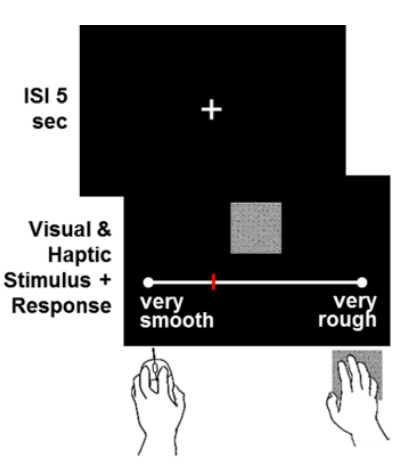

(c) Visual-haptic condition

Figure 4. Experimental paradigm. ISI=inter-stimulus interval.

\section{Data analysis}

The data were analyzed with SPSS 18 (SPSS Inc., Chicago, IL, USA). The width of the VAS for roughness scores was set to 800 pixels. The scores of all subjects were linearly transformed to a value range between 0 and 1 in order to simplify the interpretation of the results. The range of the scale was used for the transformation rather than the actual minimum and maximum ratings of the subjects in order to retain possible differences between subjects and modality conditions. These transformed values were used for all subsequent analyses. The level of significance was set to $\alpha=0.05$ for all analyses.

\section{EXPERIMENT 1}

The first experiment aimed at investigating roughness perception of unfamiliar dot pattern textures in three sensory modality conditions. Based on inconsistent findings in the literature (Connor et al., 1990; Dépeault et al., 2009; Gescheider et al., 2005; Meftah et al., 2000; Merabet et al., 2004, 2007), we addressed the question whether perceived surface roughness is better represented by a linear or bi-exponential function of inter-element spacing. Furthermore, as previous studies showed that visual and haptic information might be weighted differently in texture perception (Guest and Spence, 2003a; Heller, 1989; Lederman et al., 1986), we asked whether roughness perception changes with the kind of sensory information available as well as with the first sensory experience with the stimulus material. 


\section{METHOD}

\section{Participants}

Thirty subjects, 18 women and 12 men, mean age 28.5 years, participated in this experiment. Twenty-six of the participants were righthanded.

\section{Stimuli and apparatus}

In this experiment 29 textures were presented. The mean center-tocenter dot spacing of the stimuli ranged from 1.50 to $8.50 \mathrm{~mm}$ and increased in steps of $0.25 \mathrm{~mm}$.

\section{Procedure}

The roughness estimation task was performed for three different modality conditions: visual, haptic and a combined visual-haptic condition. The order of conditions was counter-balanced across subjects and all textures were presented three times in each condition. Since the 29 stimuli could not be arranged on a single plate due to practical limitations with respect to the diameter of the plate, 15 stimuli were placed on one presentation plate and the other 14 on a second one. The assignment of the 29 stimuli to the plates was random and changed for each subject. During practice textures with an inter-dot spacing of 1.50, 3.25, 5.00, 6.75 and $8.50 \mathrm{~mm}$ were presented once.

\section{Data analysis}

One aim of this study was to reveal the direction of the sensory information bias toward haptic or visual information for the bimodal roughness estimates. To that end a mixed-design analysis of variance (ANOVA) with the within-subject factors modality (3) and inter-dot spacing (29) and the betweensubject factor first modality condition (3) was employed for the roughness scores. The Greenhouse-Geisser correction was applied whenever sphericity could not be assumed. Furthermore, a roughness function for each subject and sensory modality was modeled by fitting a linear and bi-exponential function to the data collapsed over all repetitions. The bi-exponential model was represented by the following equation:

$r(d)=\beta 0+e^{(-\beta 2(d-\beta 1))}-e^{(-\beta 3(d-\beta 1))}$ 
where $r(d)$ is the roughness score as a bi-exponential function of inter-dot distance and $\beta 0$ to $\beta 3$ are constant terms. The first two constants $\beta 0$ and $\beta 1$ model the vertical and horizontal offset of the function while $\beta 2$ and $\beta 3$ model the shape of both exponentials. The benefit of this function over a negative quadratic function is the possibility to model asymmetric profiles of the psychophysical roughness function as explained in the introduction. Both models were compared for each condition and subject with the corrected version of Akaike Information Criterion (AICc). The AIC is a model selection method that identifies the best descriptive model for the data within a set of a priori considered models. This method was chosen because it can be applied to the comparison of non-nested models (Burnham and Anderson, 2004; Wagenmakers and Farrell, 2004) and takes the number of included parameters into account. Furthermore, a small-sample corrected version of this criterion is available (AICc) and was used for the present data as the number of data points divided by the number of free parameters is smaller than 40 , which was previously recommended as the approximated minimum value for using the uncorrected version (Burnham and Anderson, 2004). Akaike weights $\left(\mathrm{w}_{\mathrm{i}}\right)$, representing the relative probability that model $\mathrm{i}$ is the best model for the data under consideration of the set of models, were used to decide in favor of the biexponential model, when the probability was above $50 \%$.

\section{RESULTS}

The graphs in Figure 5(a) suggest that roughness judged by vision is on average attenuated in comparison to the haptic and visual-haptic judgments. This influence of modality on roughness estimation was supported by a significant main effect of the within-subject factor modality in the mixed-design ANOVA of the roughness scores, $\mathrm{F}(1.72,46.37)=12.45, \mathrm{p}<0.001$, partial $\eta^{2}=0.32$. Post hoc paired t-tests confirmed that textures were judged significantly less rough in the visual condition than in the haptic condition, mean difference: $-0.09, \mathrm{p}<0.001$, and compared to the bimodal condition, mean difference: $-0.07, \mathrm{p}<0.01$. This was not true for the direct comparison of the haptic and bimodal condition, mean difference: $0.02, \mathrm{p}=0.83$.

Furthermore, the main effect of the within-subject factor inter-dot spacing, $F(1.79,48.42)=15.99, p<0.001$, partial $\eta^{2}=0.37$, and the Modality $\times$ Inter-Dot Spacing interaction, $F(5.21,140.71)=10.26, p<0.001$, partial $\eta^{2}=0.28$ were significant. 

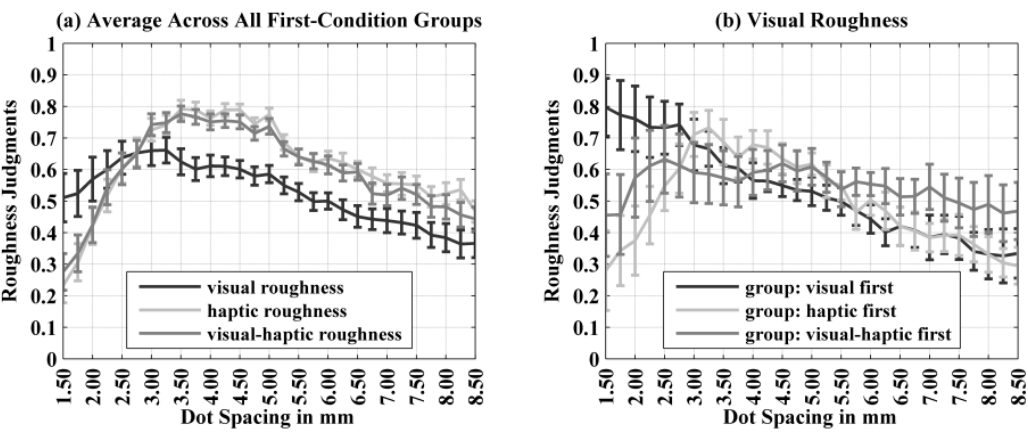

(c) Haptic Roughness
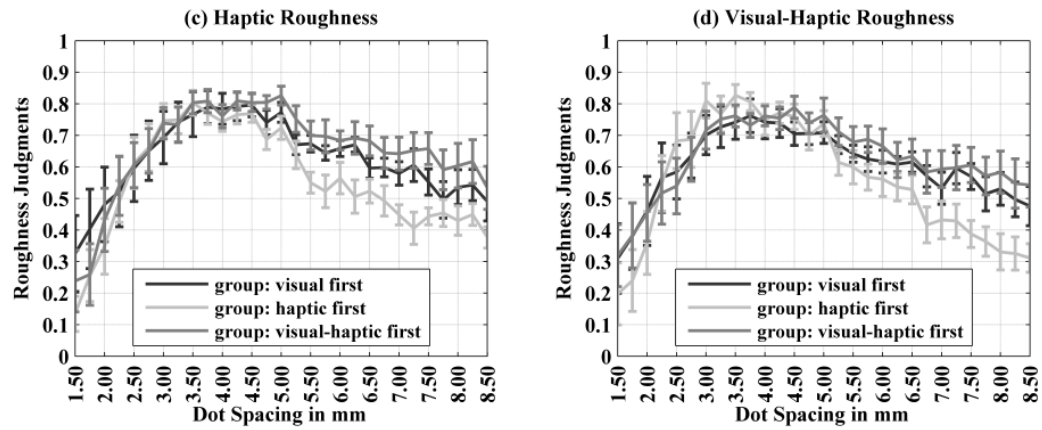

Figure 5. Mean roughness judgments in Experiment 1, averaged across all first condition groups (a), and presented separately for subjects who judged textures first by vision only, first by haptic information, or first by visual and haptic information combined in the visual (b), haptic (c) and visual-haptic condition (d). Bars represent the standard error of the mean (SEM).

This two-way interaction is also implied by Figure 5(a) showing that visual roughness exceeds roughness perceived by touch and bimodally for small inter-dot spacings while this effect is reversed for bigger dot distances. Based on the literature showing that perceived roughness peaks at about $3 \mathrm{~mm}$ interdot spacing, we used this cut off point to pool roughness judgments $\leq 3 \mathrm{~mm}$ inter-dot spacing and data acquired for textures $>3 \mathrm{~mm}$ inter-dot spacing into two groups. In post hoc contrasts all modality conditions were compared for the group of small and big inter-dot distances in order to describe the two-way interaction further. These tests confirmed partially what is indicated by Figure 5(a), namely significantly higher visual roughness scores for smaller inter-dot spacings, mean difference between the visual and haptic condition: 0.69, $\mathrm{p}<0.05$, but attenuated visual roughness scores for bigger inter-dot spacings, mean difference between the visual and haptic condition: $-3.15, \mathrm{p}<0.001$ and mean difference between the visual and bimodal condition: $-2.58, \mathrm{p}<0.001$. All other comparisons were not significant. The mixed-design ANOVA also showed a significant three-way interaction of Modality $\times$ Inter-Dot Spacing $\times$ First Condition, $F(10.42,140.71)=2.15, p<0.05$, partial $\eta^{2}=0.14$. Figure $5(b)$ 
suggests that this interaction is caused by a difference in visually perceived roughness between subjects who experienced the textures first by vision as compared to all other subjects, but for small inter-dot spacings only. Figure 5(c)-(d) indicates further that subjects who evaluated the textures first in the haptic condition show attenuated haptic and bimodal roughness scores as compared to both other first condition groups. In order to test the cause of this interaction we pooled the data into the same small and big dot distance groups as described above and compared in post-hoc contrasts the roughness estimates between all first condition groups separately for all modality conditions. These post hoc tests confirmed for small inter-dot spacings a significant difference in estimated visual roughness between subjects who experienced the textures first in the visual and first in the haptic condition, mean difference: 1.90, $<<0.05$. Haptic roughness judgments of bigger inter-dot spacings were on average attenuated when subjects experienced the textures first by touch, mean difference between haptic and visual first condition group: $-1.68, \mathrm{p}<0.05$ and mean difference between haptic and visual-haptic first condition group: -2.68 , $\mathrm{p}<0.005$. All other post hoc contrasts did not reach significance.

Table 1 summarizes the results of fitting a linear and bi-exponential model to the data of each subject and condition. In total, 12 out of 30 subjects showed a linear relationship between visual roughness estimates and inter-dot spacing of the textures. Five out of those subjects evaluated the textures first by vision, three first by touch and four first in the visual-haptic condition. In contrast, in the haptic condition only three out of the entire sample of 30 subjects showed a linear relationship between inter-dot spacing and roughness estimates (two subjects with the visual condition first and one with the bimodal condition first). This was also true for five subjects in the visual-haptic condition, two of whom were first confronted with the visual condition, two with the haptic condition and one with the bimodal condition. 
Table 1. Summary of the model comparisons of the linear and bi-exponential model for all subjects and conditions (Experiment 1)

\begin{tabular}{|c|c|c|c|c|}
\hline $\begin{array}{l}\text { First } \\
\text { condition }\end{array}$ & $\begin{array}{l}{ }^{\mathrm{a}} \text { No. of } \\
\text { subjects }\end{array}$ & $\begin{array}{l}{ }^{\mathrm{b}} \text { Range of } \\
\mathrm{R}^{2} \text { linear }\end{array}$ & $\begin{array}{l}{ }^{\mathrm{b}} \text { Range of } \\
\mathrm{R}^{2} \text { bi- } \\
\text { exponential }\end{array}$ & $\begin{array}{l}\text { Range of } \mathrm{w}_{\mathrm{i}} \\
\text { (AICc) bi- } \\
\text { exponential }\end{array}$ \\
\hline
\end{tabular}

Visual condition

Linear model selected

$\begin{array}{lllll}\text { v } & 5 & 0.81-0.96 & 0.82-0.97 & 0-32 \% \\ \text { h } & 3 & 0.90-0.95 & 0.90-0.94 & 1-32 \% \\ \text { vh } & 4 & 0.92-0.97 & 0.89-0.96 & 0-31 \%\end{array}$

Bi-exponential model selected

$\begin{array}{lllll}\mathrm{v} & 5 & 0.85-0.96 & 0.96-0.98 & 98-100 \% \\ \mathrm{~h} & 7 & 0.02-0.43 & 0.54-0.85 & 88-100 \% \\ \mathrm{vh} & 6 & 0.15-0.92 & 0.81-0.95 & 84-100 \%\end{array}$

Haptic condition

Linear model selected

$\begin{array}{lcccc}\mathrm{v} & 2 & 0.89-0.93 & 0.83-0.88 & 0 \% \\ \mathrm{~h} & 0 & - & - & - \\ \mathrm{vh} & 1 & 0.89 & 0.89 & 7 \%\end{array}$. $^{-}$

Bi-exponential model selected

$\begin{array}{lllll}\mathrm{v} & 8 & 0.04-0.97 & 0.66-0.99 & 100 \% \\ \mathrm{~h} & 10 & 0.00-0.69 & 0.70-0.90 & 100 \% \\ \mathrm{vh} & 9 & 0.00-0.72 & 0.75-0.93 & 100 \%\end{array}$

Visual-haptic condition

Linear model selected

$\begin{array}{lllll}\text { v } & 2 & 0.77-0.89 & 0.80-0.89 & 4-25 \% \\ \text { h } & 2 & 0.76-0.89 & 0.78-0.86 & 0-15 \% \\ \text { vh } & 1 & 0.94 & 0.94 & 12 \%\end{array}$

Bi-exponential model selected

$\begin{array}{lllll}\text { v } & 8 & 0.06-0.95 & 0.78-0.97 & 100 \% \\ \text { h } & 8 & 0.00-0.35 & 0.69-0.90 & 100 \% \\ \text { vh } & 9 & 0.00-0.66 & 0.56-0.91 & 100 \%\end{array}$

Note. Model decision was based on $\mathrm{w}_{\mathrm{i}}(\mathrm{AICc}) . \mathrm{v}=$ visual, $\mathrm{h}=$ haptic, $\mathrm{vh}=$ visual-haptic .

${ }^{a}$ There is a total of 10 subjects in each first condition group.

${ }^{\mathrm{b}} \mathrm{R}^{2}$ coefficient of determination. 


\section{DISCUSSION}

Previous research failed to show a difference between visual and haptic roughness estimation of abrasive paper (Lederman and Abbott, 1981). The results of the present study extend those earlier findings for a set of unfamiliar textural stimuli by showing that roughness estimates were on average slightly attenuated when only visual information of the textures was available.

Haptic and bimodal roughness perception of unfamiliar textural stimuli appears to be best described by a bi-exponential function, with a steep rise up to an inter-dot spacing of about 3-4 mm and a slighter decrease for textures with bigger dot spacing. Klatzky and Lederman (1999) found a similar increase in perceived roughness for haptic texture exploration up to $3.50 \mathrm{~mm}$ dot spacing with the bare finger. Despite the close resemblance of the findings for closely spaced dot patterns, there are several noteworthy differences in the design and analysis of Klatzky and Lederman's (1999) study. First, their maximum interelement distance in the stimulus pool was $3.50 \mathrm{~mm}$. Therefore, they could only estimate a theoretical peak based on the fitted function, which was about 4.8 $\mathrm{mm}$ for the bare finger. However, this theoretical peak might differ from empirical values. By extending the stimulus range to textures with bigger interdot separations, other studies empirically determined the peak to be between 3 and $4 \mathrm{~mm}$ (Connor et al., 1990; Gescheider et al., 2005; Merabet et al., 2007), which is consistent with our haptic results. Furthermore, Klatzky and Lederman transformed their data to $\log -\log$ space before applying a quadratic function. The fit of a quadratic function to log-log transformed roughness data of dot patterns should be comparable to the fit of the bi-exponential function to only linearly transformed data in the present study. We decided to use only linear transformations on our data, because we wanted to test against a linear model and because data presentation in plots with linear scales is more intuitive. Our results indicate that the same inverted U-shape function can be generalized to bimodal visual-haptic roughness perception of unfamiliar dot pattern textures. In contrast to the haptic and bimodal condition, visual roughness perception showed a trend for a more linear relationship with respect to inter-element spacing of textures, as evidenced by the model fitting results as well as the significant interaction of modality and dot distance in the ANOVA.

The different results for the visual modality might be explained by a difference in spatial resolution. It is known that fine spatial detail is better resolved by the eye than by the skin (Lederman and Klatzky, 2009). When touching textures with very small inter-dot spacing the finger pads might not be able to resolve the single elements due to the restricted compliance of the skin resulting in a relatively smooth texture percept. For the same small inter-dot spacing, the eye would be expected to resolve the single elements. Hence, the textures might have appeared rough to the eye because of the big number of dots and the small spacing between them. With increasing inter-dot distance, the density of elements on the surface decreases which resulted in a linear decrease 
in visually perceived roughness. If this line of reasoning applies, we would expect to find a linear relationship between inter-dot spacing and roughness perception for all subjects in the visual condition. However, the trend for a linear relationship between inter-element spacing and visual roughness estimations is weaker for subjects who experienced the stimuli first in a condition with haptic input, which is also represented by a significant interaction of modality, spacing and first condition in the ANOVA. This might indicate that subjects, who had a haptic anchor point to rely on, provided by previous haptic experience with the textures in the haptic and/or bimodal condition, tended to imagine how the visually presented textures would feel when being touched. In the remaining text, we shall refer to this imagery of the somatosensory experience related to the act of touching the textures as haptic imagery, which could be interpreted as a calibration of visual roughness judgments to haptic roughness perception. Such a calibration would be in line with the general notion of people that roughness is better evaluated by touch than by vision as, for example, indicated in a small survey with ten individuals by Lederman et al. (1986). This application of a lower resolution haptic filter on the visual roughness estimates is particularly beneficial when individuals are asked to combine both visual and haptic information in a single bimodal roughness rating. Choosing a haptic imagery strategy promotes the development of a consistent roughness concept between modalities in favor of multisensory judgments. During debriefing some of our subjects indeed reported that they used haptic imagery in the visual condition.

The general superiority of the bi-exponential model in the haptic and bimodal condition vs. the increased incidence of the linear model in the visual condition supports the findings from previous studies (Heller, 1982; Lederman et al., 1986), showing that visual-haptic roughness judgments of textures are in general more influenced by haptic than by visual information. This is further confirmed by our finding that the difference between bimodal and unimodal haptic roughness estimates is negligible, while this is not true for the comparison of visual and bimodal roughness judgments. The consistency of our results with previous findings indicates that the haptic percept receives a stronger weight, possibly because it incorporates information about the interaction of the texture and skin, which might be relevant for object manipulation.

\section{EXPERIMENT 2}

The second experiment was designed to test whether the results of the first experiment would generalize to natural viewing conditions. In the first experiment visual textures were presented on a monitor. Despite the fact that the visual stimuli were designed to be as similar as possible to the real textures, it is conceivable that unintended differences between the haptic and visual stimulus 
material influenced the results of experiment 1. Furthermore, it is known that the integration of multisensory information is optimal when the information is presented in a temporarily synchronous and spatially coincidental way, indicating that the information comes from a common source (Helbig and Ernst, 2007a, b). In the first experiment subjects were told that the stimuli presented on the screen were the same stimuli they touched. Nevertheless, the discrepant location of the visual and haptic information might have influenced cue integration across sensory modalities. Furthermore, during the exploration of the textures the hand of the subject was hidden by a cloth curtain in all three modality conditions of experiment 1 . However, Heller (1982) suggested that the visibility of the hand can influence bimodal performance. He argued that visual guidance of tactual exploration has a positive effect on bimodal texture discrimination, improving bimodal versus unimodal performance in his study. In order to account for the possibility that results of experiment 1 were influenced by the virtual presentation of the textures on a monitor, the same experiment was repeated with a more natural viewing condition.

\section{METHOD}

\section{Participants}

Eighteen volunteers, all right handed, seven males and 11 females, mean age 23.2 years, participated in this study.

\section{Stimuli and apparatus}

The same stimulus material and apparatus as described in experiment 1 were used. The experiment was conducted in a room without windows and with fluorescent lamps on the ceiling. All lights were switched on so that the room was evenly lit, equaling the lighting conditions in a normal office environment.

\section{Procedure}

Subjects performed the same roughness estimation task as described in experiment 1 . In experiment 2 , however, the same real textures were used for the haptic, visual and bimodal condition, all presented on a wooden circular board. Furthermore, subjects were able to see their own hand in the bimodal condition. 


\section{Data analysis}

The same analyses as in experiment 1 were performed. These included a mixed-design ANOVA with the within-subject factors modality (3) and interdot spacing (29) and the between-subject factor first modality condition (3) as well as the model fitting of the linear and bi-exponential function. When sphericity was violated the Greenhouse Geisser adjustment for degrees of freedom was used in the ANOVA.

\section{RESULTS}

The mixed-design ANOVA showed a significant main effect of the within-subject factor inter-dot spacing, $\mathrm{F}(1.67,25.02)=23.95$, $\mathrm{p}<0.001$, partial $\eta^{2}=0.62$. However, the main effects of the within-subject factor modality and the between-subject factor first modality condition did not reach significance, $\mathrm{F}(1.41,21.17)=0.32, \mathrm{p}=0.65$, partial $\eta^{2}=0.02$ and $\mathrm{F}(2,15)=0.63, \mathrm{p}=0.55$, partial $\eta^{2}=0.08$, respectively.

The plot in Figure 6(a) implies that visually perceived roughness of textures with small inter-dot spacings is on average higher as compared to haptic and visual-haptic roughness perception, while the opposite seems to be true for inter-dot spacings above $3 \mathrm{~mm}$. This was confirmed by a significant two-way interaction of Modality $\times$ Inter-Dot Spacing, $F(6.58,98.65)=11.59$, $\mathrm{p}<0.001$, partial $\eta^{2}=0.44$. Post-hoc contrasts comparing all modality conditions for the pooled data of textures $\leq 3 \mathrm{~mm}$ and for roughness estimates $>3 \mathrm{~mm}$ showed similar effects as in experiment 1 , namely significantly higher visual judgments in the group of small inter-dot spacings, mean difference between the visual and haptic condition: $1.29, \mathrm{p}<0.001$ and mean difference between the visual and bimodal condition: $1.13, \mathrm{p}<0.001$. The effect was reversed for roughness judgments in the group of textures with big inter-dot spacing, mean difference between the visual and haptic condition: $-1.21, \mathrm{p}<0.05$ and mean difference between the visual and bimodal condition: $-1.35, \mathrm{p}<0.005$. The mixed-design ANOVA revealed also a significant interaction of the withinsubject factor sensory modality and the between-subject factor first modality condition, $\mathrm{F}(2.82,21.17)=6.25, \mathrm{p}<0.005$, partial $\eta^{2}=0.46$, with haptic roughness scores being on average lower than bimodal roughness judgments when textures were judged first in the haptic condition, mean difference: $-0.07, \mathrm{p}<0.005$, and higher when textures were first experienced in the bimodal condition, mean difference: 0.05, $<<0.05$. The two-way interaction of First Condition $\times$ Inter-Dot Spacing did not reach significance in the mixed-design ANOVA, F(3.34, 25.02) $=1.22, \mathrm{p}=0.32$, partial $\eta^{2}=0.14$. 
(a) Average Across All First-Condition Groups

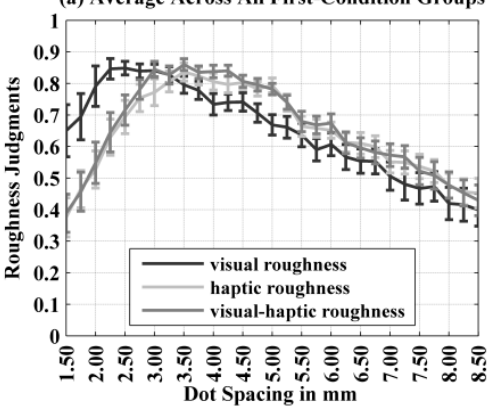

(c) Haptic Roughness

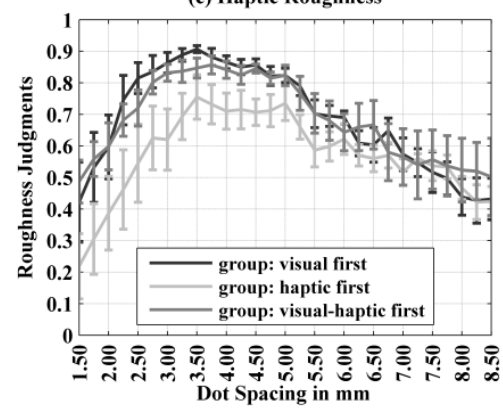

(b) Visual Roughness

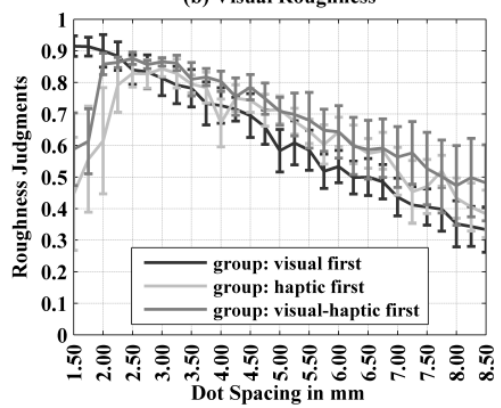

(d) Visual-Haptic Roughness

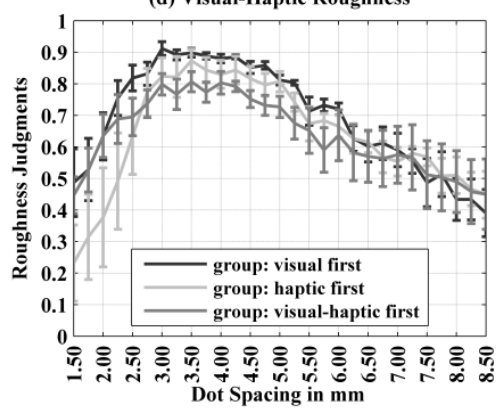

Figure 6. Mean roughness judgments in Experiment 2, averaged across all first condition groups (a), and presented separately for subjects who judged textures first by vision only, first by haptic information, or first by visual and haptic information combined in the visual (b), haptic (c) and visual-haptic condition (d). Bars represent the standard error of the mean (SEM).

Furthermore, there was a significant three-way interaction of Modality $\times$ First Condition $\times$ Inter-Dot Spacing, $F(13.15,98.65)=1.91, p<0.05$, partial $\eta^{2}=0.20$. The plot in Figure 6(b) suggests that this interaction is caused by a difference in visually perceived roughness of textures with inter-dot spacings $\leq 2.50 \mathrm{~mm}$ depending on the modality with which subjects experienced the textures first. Additionally, there seems to be an effect of the factor first condition on haptic roughness perception for textures with intermediate interdot spacing $(2.75-5.00 \mathrm{~mm})$ as presented in Figure 6(c). We investigated these hypotheses by pooling the roughness judgments into three different groups of inter-dot spacing, i.e. $1.50-2.50 \mathrm{~mm}, 2.75-5.00 \mathrm{~mm}, 5.25 \mathrm{~mm}-8.50 \mathrm{~mm}$, and by comparing all first conditions within these groups separately for the different sensory modalities. The post-hoc contrasts confirmed significantly higher visual roughness estimates for inter-dot spacings $\leq 2.50 \mathrm{~mm}$ when subjects experienced the textures first in the visual as compared to the haptic condition, mean difference: $1.21, \mathrm{p}<0.05$. In the haptic condition roughness judgments were attenuated for textures with intermediate inter-dot spacings when subjects experienced the textures first by touch, mean difference between the haptic and 
visual first condition group: $-1.58, \mathrm{p}<0.01$ and mean difference between the haptic and bimodal first condition group: $-1.32, \mathrm{p}<0.05$. There was also a significant effect of first condition on bimodal roughness judgments of textures with intermediate dot distances, i.e. with attenuated scores for subjects who experienced the textures first in the bimodal as compared to the visual condition, mean difference: $-1.02, \mathrm{p}<0.05$.

The model fitting results are comparable to the findings of experiment 1 in which the visual textures were presented on a monitor. Only two out of all 18 subjects showed a better fit for the linear model in the haptic condition, with one having experienced the textures first in the visual condition and one in the visual-haptic condition. The same two subjects also showed a better fit for the linear model in the visual-haptic condition, while the bi-exponential model was superior for all other subjects. Only in the visual condition the incidence of the linear model increased with seven out of 18 subjects. In line with the results of experiment 1, the highest incidence of the linear model can be found in the group of subjects who experienced the textures first by vision, i.e. four out of six participants, while only one subject who experienced the textures first by touch and only two subjects in the visual-haptic first condition group showed a better fit for the linear model (see Table 2).

\section{DISCUSSION}

The findings of the second experiment using natural visual textures mainly confirm the results of the first experiment in which the visual stimuli were presented on a monitor. Haptic and bimodal roughness perception were well described by a bi-exponential function of inter-dot spacing peaking at about 3-4 mm, whereas the incidence of the linear function increased for visual roughness perception, as also indicated by the interaction of modality and interdot spacing in the ANOVA. This might be caused by a different spatial resolving power of the visual and haptic system, as already discussed in experiment 1. This distribution difference of the linear and bi-exponential model for visual and haptic roughness perception supports our assumption of an influence of the spatial resolution of both senses on roughness perception of dot pattern textures. Again, there seems to be an effect of the first sensory experience with the stimulus material on visual roughness perception: there is a stronger tendency toward the bi-exponential model for visual roughness perception when subjects can refer to a haptic anchor point obtained from previous texture exploration in the haptic or bimodal condition. This effect of the first modality condition on visual roughness judgments was confirmed by the significant interaction of first condition, modality and inter-element spacing. Hence, our interpretation that individuals tend to calibrate their visual roughness estimates to haptic roughness perception when provided with haptic experience is further supported. 
Table 2. Summary of the model comparisons of the linear and bi-exponential model for all subjects and conditions (Experiment 2)

\begin{tabular}{lllll}
\hline $\begin{array}{l}\text { First } \\
\text { condition }\end{array}$ & $\begin{array}{l}{ }^{\mathrm{a}} \text { No. of } \\
\text { subjects }\end{array}$ & $\begin{array}{l}{ }^{\mathrm{b}} \text { Range of } \\
\mathrm{R}^{2} \text { linear }\end{array}$ & $\begin{array}{l}{ }^{\mathrm{b}} \text { Range of } \\
\mathrm{R}^{2} \text { bi- } \\
\text { exponential }\end{array}$ & $\begin{array}{l}\text { Range of } \mathrm{w}_{\mathrm{i}} \\
\text { (AICc) bi- } \\
\text { exponential }\end{array}$ \\
\hline
\end{tabular}

Visual condition

Linear model selected

$\begin{array}{lllll}\mathrm{v} & 4 & 0.88-0.94 & 0.88-0.90 & 0-23 \% \\ \mathrm{~h} & 1 & 0.95 & 0.88 & 0 \% \\ \mathrm{vh} & 2 & 0.92-0.95 & 0.85-0.88 & 0 \%\end{array}$

Bi-exponential model selected

$\begin{array}{lllll}\mathrm{v} & 2 & 0.92-0.95 & 0.97 & 99-100 \% \\ \mathrm{~h} & 5 & 0.08-0.95 & 0.68-0.95 & 77-100 \% \\ \mathrm{vh} & 4 & 0.01-0.28 & 0.82-0.93 & 100 \%\end{array}$

Haptic condition

Linear model selected

$\begin{array}{llccc}\mathrm{v} & 1 & 0.94 & 0.92 & 0 \% \\ \mathrm{~h} & 0 & - & - & \\ \mathrm{vh} & 1 & 0.77 & 0.73 & 1 \%\end{array}$

Bi-exponential model selected

$\begin{array}{lllll}\mathrm{v} & 5 & 0.01-0.43 & 0.78-0.91 & 100 \% \\ \mathrm{~h} & 6 & 0.06-0.55 & 0.65-0.92 & 100 \% \\ \mathrm{vh} & 5 & 0.00-0.64 & 0.74-0.92 & 84-100 \%\end{array}$

Visual-haptic condition

Linear model selected

$\begin{array}{llccc}\mathrm{v} & 1 & 0.91 & 0.86 & 100 \% \\ \mathrm{~h} & 0 & & - & - \\ \mathrm{vh} & 1 & 0.83 & 0.76 & 100 \%\end{array}$

Bi-exponential model selected

$\begin{array}{lllll}\text { v } & 5 & 0.02-0.28 & 0.73-0.87 & 100 \% \\ \text { h } & 6 & 0.03-0.67 & 0.74-0.91 & 100 \% \\ \text { vh } & 5 & 0.00-0.47 & 0.77-0.87 & 100 \%\end{array}$

Note. Model decision was based on $\mathrm{w}_{\mathrm{i}}(\mathrm{AICc}) . \mathrm{v}=$ visual, $\mathrm{h}=$ haptic, $\mathrm{vh}=$ visual-haptic.

${ }^{\mathrm{a}}$ There is a total of 6 subjects in each first condition group.

${ }^{\mathrm{b}} \mathrm{R}^{2}$ coefficient of determination. 
However, the results of the second experiment did not confirm the main effect of modality on roughness perception. We cannot rule out that this difference between the first and second experiments is caused by the different presentation modes of the visual stimuli, but the significant interaction of modality and spacing in both experiments indicates a similar trend. One possible explanation for the non-significant main effect of modality might be the relatively small effect of attenuated visual roughness scores for textures with inter-dot spacings above $3 \mathrm{~mm}$. This effect might have been cancelled out by the rather strong effect of increased visual roughness judgments for textures with smaller dot distances.

In general, the similarity of findings in experiments 1 and 2 leads to the conclusion that the presentation mode of visual stimuli has no or only a negligible influence on visual and bimodal roughness perception of dot pattern stimuli. Hence, the additional visibility of the exploring hand in the bimodal condition, as compared to the first experiment, did not change the overall results. This is in contrast to the findings of Heller (1982) who showed that the visibility of the exploring hand and not the visual texture information per se was crucial for a performance improvement in a bimodal as compared to a haptic texture discrimination task. He also reported that subjects showed a preference for haptic texture cues when visual information was available. This stronger weight of haptic information for bimodal judgments was confirmed in the present study for both virtual and natural visual presentation conditions. We did not find a change in bimodal perception between the virtual and the natural setting possibly because of the nature of the experimental task. Visual guidance of hand movements might be particularly beneficial when fine discriminations between textures are required, as in Heller's experiment for abrasive papers. Such fine relative judgments were not required from the participants in our experiment; hence no added value by visual guidance of the hand movements and therefore no change in the bimodal results are expected.

The similarity of findings in experiments 1 and 2 opens the possibility to translate the behavioral experimental design to virtual environments or to a setup with restricted viewing conditions, i.e. for future comparative neuroimaging studies. Such an approach might broaden our understanding of the neural correlates of visual-haptic bimodal roughness perception.

\section{EXPERIMENT 3}

The effect of the first sensory experience with unfamiliar textures on visual roughness perception reported in the first and second experiments is tested here in a setting in which participants are not forced to integrate visual and haptic roughness information. This is different from individuals who performed the bimodal condition last in experiments 1 and 2, in the sense that subjects in the former experiments knew that a combination of visual and haptic 
texture information in a single bimodal judgment was required. We propose that the order effect of modality conditions on visual roughness perception is primarily expected when subjects are required to combine both kinds of unimodal information to a bimodal judgment, increasing the chance of using haptic imagery for visual roughness estimation. In a setting in which haptic and visual information is processed separately only, we hypothesize that subjects will utilize the higher visual spatial resolution to judge visual roughness.

\section{METHOD}

\section{Participants}

Twenty-two paid volunteers, four males and 18 females, mean age 23.1 years, participated in this study. Twenty subjects were right handed.

\section{Stimuli and apparatus}

The set of stimuli consisted of fifteen textures, ranging from 1.50 to $8.50 \mathrm{~mm}$ inter-dot spacing, increasing in steps of $0.50 \mathrm{~mm}$. Visual stimuli were again computer-rendered images of the real textures, presented on a monitor.

\section{Procedure}

Subjects performed the same roughness estimation task as described in experiment 1 in a visual and haptic condition only. The order of conditions was counter-balanced across subjects and all textures were presented three times in each condition. The practice trials included three textures with an inter-dot distance of 1.50, 5.00 and $8.50 \mathrm{~mm}$ presented in ascending order.

\section{Data analysis}

Roughness scores were analyzed with a mixed-design ANOVA with the within-subject factors modality (2) and inter-dot spacing (15) and the betweensubject factor first modality condition (2). The Greenhouse-Geisser correction was used where appropriate. In addition, a linear and bi-exponential function was fitted to the data as described in experiment 1 . Both models were compared for each subject and condition with the corrected version of the Akaike Information Criterion. 


\section{RESULTS}

The plot of the mean roughness judgments averaged across both first condition groups (see Figure 7(a)) indicates attenuated roughness judgments in the visual as compared to the haptic condition. The mixed-design ANOVA of roughness judgments confirms this with significant main effects of the withinsubject factors modality, $\mathrm{F}(1,20)=5.18, \mathrm{p}<0.05$, partial $\eta^{2}=0.21$ and inter-dot spacing, $\mathrm{F}(1.48,29.63)=9.99, \mathrm{p}<0.005$, partial $\eta^{2}=0.33$. There is also a significant main effect of the between-subject factor first condition, $F(1$, $20)=4.56, p<0.05$, partial $\eta 2=0.19$, with subjects judging the textures on average less rough when they experienced the stimuli first by touch as compared to vision (see Figure 7(b)).
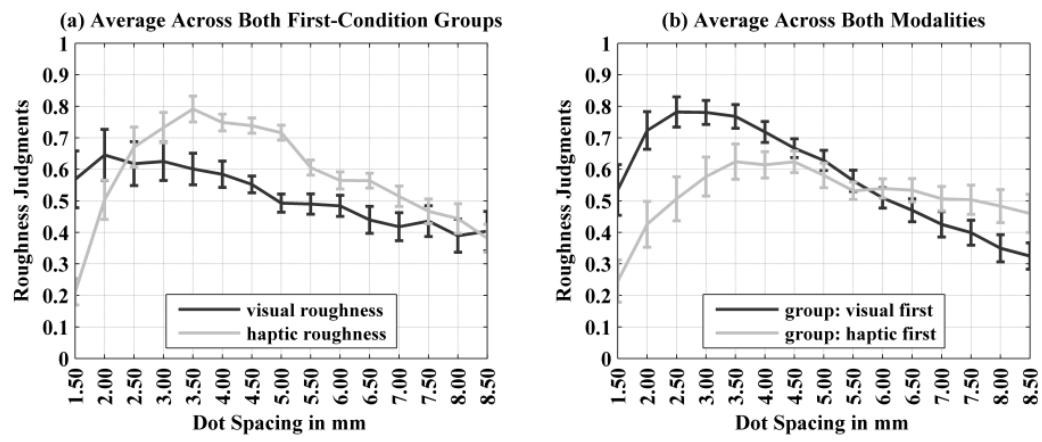

Figure 7. Mean roughness judgments in Experiment 3, averaged across both first condition groups (a) and averaged across both modality conditions (b). Bars represent the standard error of the mean (SEM).

Next to the main effects both two-way interactions of First Condition $x$ Inter-Dot Spacing, $\mathrm{F}(1.48,29.63)=5.14, \mathrm{p}<0.05$, partial $\eta^{2}=0.20$, and Sensory Modality $\times$ Inter-Dot Spacing, $\mathrm{F}(1.96,39.13)=8.95, \mathrm{p}<0.005$, partial $\eta^{2}=0.31$ reached significance. These interactions were further explored by pooling all roughness judgments of textures with inter-dot spacings $\leq 3 \mathrm{~mm}$ and dot distances $>3 \mathrm{~mm}$. Within these groups of inter-dot spacings post hoc contrasts compared roughness estimations of both modality conditions independent of the first condition factor. The results of the post-hoc tests confirmed what is implied by Figure 7(a), namely significantly lower visual roughness judgments for textures with big inter-dot spacings, mean difference: $-1.25, \mathrm{p}<0.005$ and no significant difference for small inter-dot spacings. The comparison of both first condition groups independent of the modality condition yielded a significant result for small inter-dot spacings, mean difference: $2.13, \mathrm{p}<0.01$, but not for textures with dot distances $>3 \mathrm{~mm}$ (Figure 7(b)). 
Only the three-way interaction of Modality $\times$ First Condition $\times$ InterDot Spacing did not reach significance in the mixed-design ANOVA, F(1.96, 39.13) $=1.82, \mathrm{p}=0.18$, partial $\eta^{2}=0.08$.

In line with the roughness estimation task reported in the first two experiments we found the bi-exponential model to be superior to the linear model in the haptic condition, i.e. roughness estimations of all subjects were better characterized by a bi-exponential function of inter-dot spacing. Subjects also reported for the haptic task that textures with very small and very wide inter-element spacing felt smooth while the intermediate surfaces felt rather rough. In contrast, in the visual condition, the results indicated that in 16 out of 22 participants visually perceived roughness was linearly related to inter-dot spacing, while only six subjects showed a better fit for the bi-exponential model, with three having experienced the textures first by touch and three in the visual condition. The superiority of the linear model for the visual condition was also reflected in the verbal reports of subjects. Eighteen out of the 22 participants stated that their visual roughness perception was correlated with spatial density or the number of elements on the stimuli. Seven of these subjects had experienced the textures first by touch and 11 first by vision. The three subjects that showed a better fit for the bi-exponential model in the visual condition and experienced the textures first by touch reported that they used the same rating strategy as in the haptic task. One participant could not verbalize the roughness rating strategy used. Table 3 summarizes the results of fitting both models to the data of each subject in both conditions.

\section{DISCUSSION}

The analysis of the perceived roughness scores shows that individuals judge textures to be less rough when there is only visual information available, confirming the findings of the first experiment. The curve fitting procedure for the haptic roughness judgments essentially replicates the results of the first two experiments; roughness perception of unfamiliar dot pattern stimuli can be best described as a bi-exponential function of inter-dot distance peaking for textures with about 3-4 $\mathrm{mm}$ mean center-to-center dot spacing. However, visual roughness perception was for most subjects linearly related to inter-element spacing, regardless of the first sensory experience with the stimulus material. It is possible that subjects tried to imagine how the textures would feel. However, due to their inexperience with the dot patterns in the visual-first group, participants might have failed to consider the likely decrease of skin deformation (and hence the decrease in roughness) for textures with very small dot spacings, too small for the folds of the skin to enter and to be maximally indented. This would also explain the higher incidence of the linear model in the visual-first groups in experiments 1 and 2. For subjects who experienced the textures first by touch, the haptic imagery process would lead to a bi- 
exponential roughness function in the visual condition, due to the refined haptic texture concept for this group of participants. However, the results contradict that explanation; an equally high number of subjects who first experienced the textures by touch also showed a better fit for the linear model in the visual condition of the current experiment. This indicates that haptic experience with the stimulus material has only a small or even negligible influence on visual roughness perception of unfamiliar dot pattern textures when no combination of visual and haptic information in a bimodal roughness estimate is necessary.

Table 3. Summary of the model comparisons of the linear and bi-exponential model for all subjects and conditions (Experiment 3)

\begin{tabular}{lllll}
\hline $\begin{array}{l}\text { First } \\
\text { condition }\end{array}$ & $\begin{array}{l}{ }^{\mathrm{a}} \text { No. of } \\
\text { subjects }\end{array}$ & $\begin{array}{l}{ }^{\mathrm{b}} \text { Range of } \\
\mathrm{R}^{2} \text { linear }\end{array}$ & $\begin{array}{l}{ }^{\mathrm{b}} \text { Range of } \\
\mathrm{R}^{2} \text { bi- } \\
\text { exponential }\end{array}$ & $\begin{array}{l}\text { Range of } \mathrm{w}_{\mathrm{i}} \\
\text { (AICc) bi- } \\
\text { exponential }\end{array}$ \\
\hline
\end{tabular}

Visual condition

Linear model selected

$\begin{array}{lllll}\mathrm{v} & 8 & 0.84-0.96 & 0.80-0.96 & 0-46 \% \\ \mathrm{~h} & 8 & 0.86-0.97 & 0.84-0.97 & 0-17 \%\end{array}$

Bi-exponential model selected
$\mathrm{v}$
3
$0.07-0.92$
$0.51-0.96$
$61-93 \%$
$\mathrm{h}$
3
$0.03-0.37$
$0.52-0.89$
$74-100 \%$

Haptic condition

Linear model selected

$\begin{array}{ll}\mathrm{v} & 0 \\ \mathrm{~h} & 0\end{array}$

Bi-exponential model selected

\begin{tabular}{lllll}
$\mathrm{v}$ & 11 & $0.00-0.73$ & $0.67-0.99$ & $80-100 \%$ \\
$\mathrm{~h}$ & 11 & $0.00-0.92$ & $0.51-0.97$ & $60-100 \%$ \\
\hline
\end{tabular}

Note. Model decision was based on $\mathrm{w}_{\mathrm{i}}(\mathrm{AICc}) . \mathrm{v}=$ visual, $\mathrm{h}=$ haptic .

${ }^{\mathrm{a}}$ There is a total of 11 subjects in each first condition group.

${ }^{\mathrm{b}} \mathrm{R}^{2}$ coefficient of determination.

Individuals possibly used the high spatial resolution of the visual sense to estimate roughness of unfamiliar dot pattern surfaces irrespective of previous experience with the textures. The verbal reports of almost all participants, stating that they used the number of elements and spatial density of the textures in the visual condition, further support this hypothesis. However, the fact that not all subjects showed the linear psychophysical roughness function in the 
visual condition implies that there are also individual differences in the bias toward using one sense or the other, as discussed by Guest and Spence (2003b) in the context of multisensory texture processing. It is conceivable that some subjects used haptic imagery in the visual condition to derive roughness information, resulting in a bi-exponential roughness function of inter-dot spacing just as in the haptic condition. Nevertheless, a consistent roughness concept between modalities is not necessarily helpful in a setting which does not require the combination of both sensory cues.

\section{EXPERIMENT 4}

As already discussed before, visual spatial resolution is higher than haptic spatial resolution, possibly explaining why visual roughness estimations still increase for small inter-dot spacings while haptic roughness judgments decrease. This raises the question whether visual roughness estimations decrease again for textures with very small mean center-to-center dot distances, basically representing a similar bi-exponential roughness function of interelement spacing as in the haptic modality but with a shifted peak toward a smaller spacing (see Figure 8 for a schematic representation). Hence, in the current experiment we tested the hypothesis that visual dot pattern stimuli with an inter-dot spacing below $1.50 \mathrm{~mm}$ result in a decrease in visually perceived roughness.

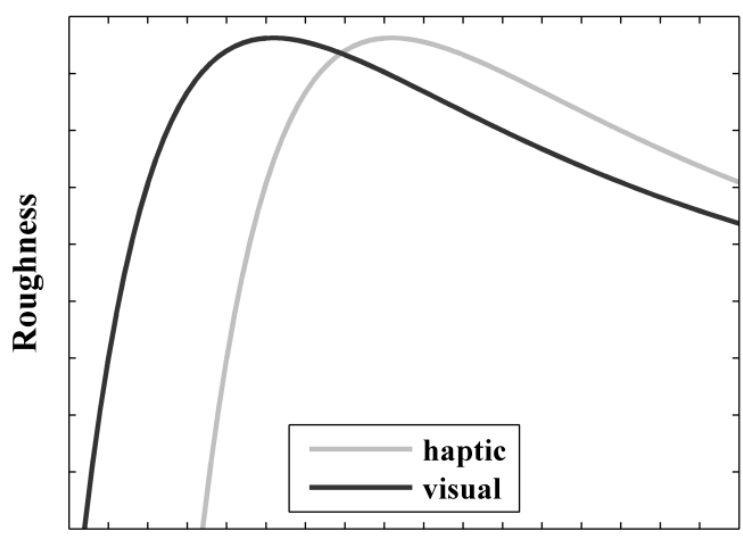

Dot Spacing

Figure 8. Model of the psychophysical function representing visual compared to haptic roughness perception. 


\section{METHOD}

\section{Participants}

Sixteen subjects, six males and 10 females with a mean age of 34.8 participated in the study.

\section{Stimuli and apparatus}

Five new stimuli were created with a mean center-to-center dot spacing of $0.25,0.50,0.75,1.00$ and $1.25 \mathrm{~mm}$, resulting in an overlap of neighboring dots and hence in finer textures. In total 34 dot pattern stimuli were presented ranging from 0.25 to $8.50 \mathrm{~mm}$ inter-dot distance and increasing in steps of 0.25 $\mathrm{mm}$.

\section{Procedure}

Subjects performed the same roughness estimation task as described in experiment 1 but in a visual condition only. All textures were presented four times and the practice run consisted of five example stimuli with an inter-dot spacing of $0.25,2.25,4.25,6.25$ and $8.50 \mathrm{~mm}$.

\section{Data analysis}

A linear and bi-exponential function was fitted to the data and both models were compared with AICc as described in experiment 1.

\section{RESULTS}

Visually perceived roughness averaged over all participants follows an almost identical bi-exponential function as depicted for roughness experienced by touch in the previous experiments (see Figure 9). The only difference to the haptic function is an earlier peak for textures with an inter-dot spacing of about $1.25-1.75 \mathrm{~mm}$. The superiority of the bi-exponential model for this set of visual textures was confirmed by the number of subjects who showed a better fit for the bi-exponential compared to the linear curve, i.e. 15 versus 1 (see Table 4). 
Table 4. Summary of the model comparisons of the linear and bi-exponential model for visually perceived roughness of all subjects (Experiment 4)

\begin{tabular}{lllll}
\hline Model selected & $\begin{array}{l}{ }^{\mathrm{a}} \text { No. of } \\
\text { subjects }\end{array}$ & $\begin{array}{l}{ }^{\mathrm{b}} \text { Range of } \\
\mathrm{R}^{2} \text { linear }\end{array}$ & $\begin{array}{l}{ }^{\mathrm{b}} \text { Range of } \\
\mathrm{R}^{2} \text { bi- } \\
\text { exponential }\end{array}$ & $\begin{array}{l}\text { Range of } \mathrm{w}_{\mathrm{i}} \\
\text { (AICc) bi- } \\
\text { exponential }\end{array}$ \\
\hline Linear & 1 & 0.98 & 0.98 & $31 \%$ \\
Bi-exponential & 15 & $0.00-0.91$ & $0.59-0.99$ & $100 \%$ \\
\hline
\end{tabular}

Note. Model decision was based on $\mathrm{w}_{\mathrm{i}}$ (AICc).

${ }^{\mathrm{a}}$ There is a total of 16 subjects.

${ }^{\mathrm{b}} \mathrm{R}^{2}$ coefficient of determination.

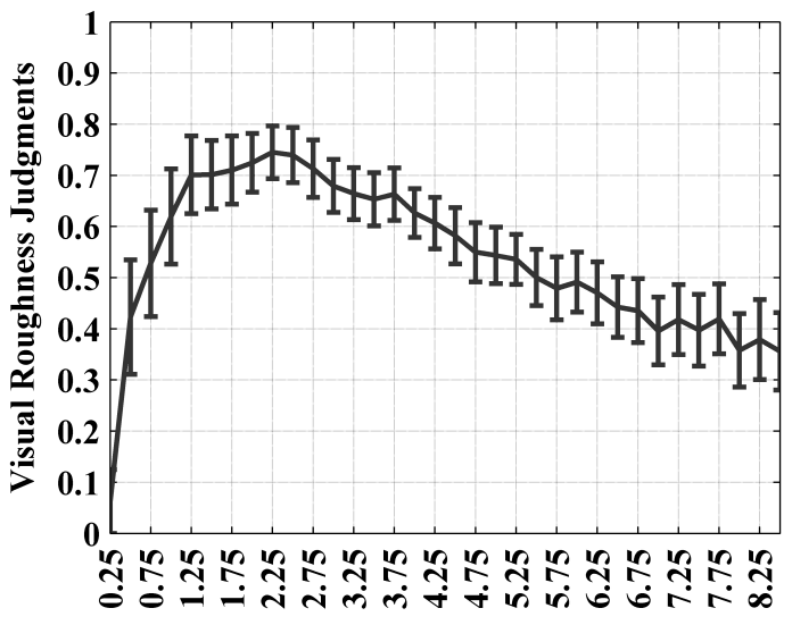

Dot Spacing in $\mathbf{m m}$

Figure 9. Mean visual roughness judgments in Experiment 4. Bars represent the standard error of the mean (SEM).

\section{DISCUSSION}

The results of this experiment show that visual roughness perception of unfamiliar dot pattern stimuli ranging from 0.25 to $8.50 \mathrm{~mm}$ inter-element spacing is well characterized by a bi-exponential function peaking for textures with an inter-dot spacing of about $1.25-1.75 \mathrm{~mm}$. This indicates that the haptic and visual psychophysical function for roughness perception of unfamiliar dot patterns can be characterized by the same curve but differs in the location of the maximum, likely due to a difference in the spatial resolving power between both modalities. We do not claim that spatial resolution is the only factor limiting visual roughness perception of these textures. Certainly, contrast sensitivity also contributes to a decline in roughness perception, especially for 
textures below $0.75 \mathrm{~mm}$ inter-dot spacing for which a big overlap between texture elements is present and hence a decrease in local contrast. However, visual roughness perception seems to decrease already for textures with an inter-dot spacing of 0.75 and $1.00 \mathrm{~mm}$ for which contrast sensitivity as the limiting factor seems rather unlikely.

\section{EXPERIMENT 5}

Note that there is another explanation for the results in experiment 4; due to a missing scale reference for size comparison of the visual stimuli, subjects might have judged the size of the textures differently than they would have in a natural haptic roughness exploration condition, resulting in a different peak location of the visual roughness function. Therefore, we presented the same textures again to a new group of observers, but this time in four different scale reference conditions, in order to test the alternative hypothesis that visual roughness perception of dot pattern textures is influenced by the scale context when stimuli are presented on a monitor.

\section{METHOD}

\section{Participants}

Twelve subjects, five males and seven females with a mean age of 21.5 participated in the study.

\section{Stimuli and apparatus}

The stimulus set was the same as in experiment 4. However, in addition to a no-reference condition in which no scale reference was presented, there were three other conditions showing a picture of a hand next to the textures in varying sizes, i.e. with a natural size ratio of hand and stimulus, a hand with $50 \%$ and a hand with $150 \%$ of the natural size ratio (see Figure 10).

\section{Procedure}

Subjects performed the same visual roughness estimation task as described in experiment 4. Participants were informed that they would be presented with one texture at a time and were asked to evaluate the roughness of this texture. No specific instruction on how to use the reference picture next to 
the texture was provided. The order of conditions was counter-balanced across subjects and all textures were presented randomly, three times in each condition. The practice run, in the beginning of the experiment, consisted of five example stimuli with an inter-dot spacing of $0.25,2.25,4.25,6.25$ and $8.50 \mathrm{~mm}$ and no scale reference.

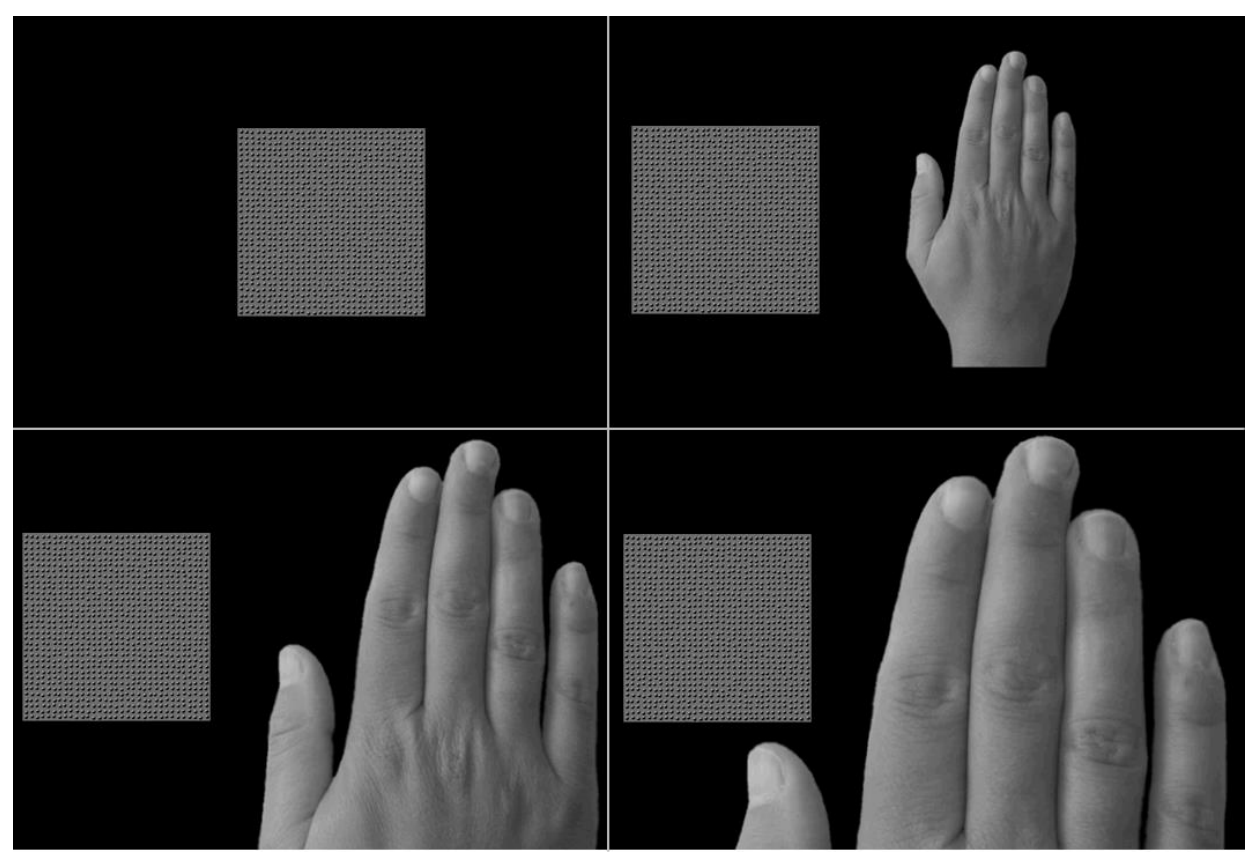

Figure 10. Examples of the four different scale reference conditions in Experiment 5. Top right: no scale reference, bottom right: natural size ratio, top left: $50 \%$ of natural size ratio, bottom left: $150 \%$ of natural size ratio.

\section{Data analysis}

Roughness scores were analyzed with a repeated measures ANOVA with the within-subject factors scale reference condition (4) and inter-dot spacing (34). The Greenhouse-Geisser adjustment for degrees of freedom was used when the sphericity assumption was violated. Additionally, a linear and biexponential function was fitted to the data and both models were compared separately for all four scale reference conditions with AICc.

\section{RESULTS}

The repeated measures ANOVA showed only a main effect of the within-subject factor inter-dot spacing $\mathrm{F}(1.74,19.16)=14.36, \mathrm{p}<0.001$, partial 
$\eta^{2}=0.57$. Neither the main effect of the factor scale reference nor the Scale Reference $\times$ Inter-Dot Spacing interaction reached significance, $F(2.35$, 25.87) $=0.61, \mathrm{p}=0.58$, partial $\eta^{2}=0.05$ and $\mathrm{F}(4.79,52.71)=1.03$, $\mathrm{p}=0.41$, partial $\eta^{2}=0.09$, respectively. The model fitting confirms the results of the ANOVA showing that there is no effect of scale context on visual roughness perception in that experiment (see Figure 11). All 12 subjects showed a better fit for the biexponential as compared to the linear model in all four scale reference conditions (see Table 5).

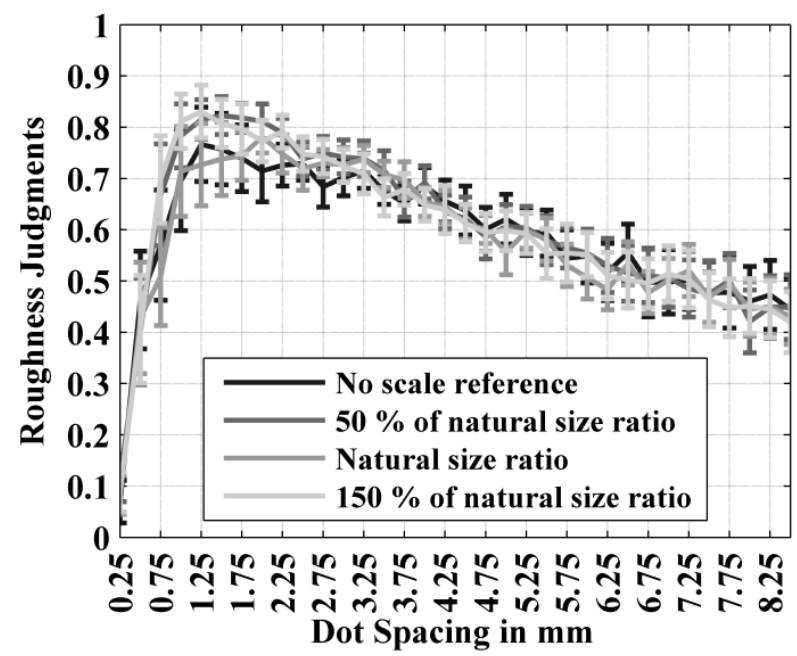

Figure 11. Mean visual roughness judgments for all four scale reference conditions in Experiment 5. Bars represent the standard error of the mean (SEM).

\section{DISCUSSION}

The results of the fifth experiment indicate that visual roughness perception of dot pattern textures is invariant to changes in scale context when stimuli are presented on a monitor. Visual roughness perception of these unfamiliar textures was well described by a bi-exponential function peaking at about 1.25-1.75 mm inter-dot spacing regardless of the scale context in which the textures were presented. We are aware that we tested only a limited subset of scale contexts and that by choosing more extreme size differences between textures and the hand, the results might have been different. However, this experiment aimed at investigating whether size mis-calculations of the virtual visual stimuli in comparison to the real textures might have resulted in the change of the maximum of the visual roughness function toward smaller interdot spacings in experiment 4 . By testing these naturalistic size differences between the textures and the hand, we believe we were able to show that this alternative explanation is rather unlikely. Therefore, our initial interpretation of 
the findings seems to hold, i.e. the difference in the peak location of the visual and haptic roughness function of dot pattern textures can be well explained by the better spatial resolution of the visual system.

Table 5. Summary of the model comparisons of the linear and bi-exponential model for all subjects and conditions (Experiment 5)

\begin{tabular}{|c|c|c|c|c|}
\hline Condition & $\begin{array}{l}{ }^{\mathrm{a}} \text { No. of } \\
\text { subjects }\end{array}$ & $\begin{array}{l}{ }^{\mathrm{b}} \text { Range of } \\
\mathrm{R}^{2} \text { linear }\end{array}$ & $\begin{array}{l}{ }^{\mathrm{b}} \text { Range of } \\
\mathrm{R}^{2} \text { bi- } \\
\text { exponential }\end{array}$ & $\begin{array}{l}\text { Range of } w_{i} \\
\text { (AICc) bi- } \\
\text { exponential }\end{array}$ \\
\hline \multicolumn{5}{|l|}{ Linear model selected } \\
\hline $\begin{array}{l}\text { No scale } \\
\text { reference }\end{array}$ & 0 & - & - & - \\
\hline $\begin{array}{l}\text { Natural size } \\
\text { ratio }\end{array}$ & 0 & - & - & - \\
\hline $\begin{array}{l}150 \% \text { of natural } \\
\text { size ratio }\end{array}$ & 0 & - & - & - \\
\hline $\begin{array}{l}50 \% \text { of natural } \\
\text { size ratio }\end{array}$ & 0 & - & - & - \\
\hline \multicolumn{5}{|c|}{ Bi-exponential model selected } \\
\hline $\begin{array}{l}\text { No scale } \\
\text { reference }\end{array}$ & 12 & $0.01-0.83$ & $0.42-0.95$ & $100 \%$ \\
\hline $\begin{array}{l}\text { Natural size } \\
\text { ratio }\end{array}$ & 12 & $0.00-0.76$ & $0.51-0.97$ & $100 \%$ \\
\hline $\begin{array}{l}150 \% \text { of natural } \\
\text { size ratio }\end{array}$ & 12 & $0.04-0.88$ & $0.76-0.96$ & $100 \%$ \\
\hline $\begin{array}{l}50 \% \text { of natural } \\
\text { size ratio }\end{array}$ & 12 & $0.00-0.76$ & $0.62-0.97$ & $100 \%$ \\
\hline
\end{tabular}

Note. Model decision was based on $\mathrm{w}_{\mathrm{i}}$ (AICc).

${ }^{\mathrm{a}}$ There is a total of 12 subjects.

${ }^{\mathrm{b}} \mathrm{R}^{2}$ coefficient of determination.

\section{GENERAL DISCUSSION}

In the experiments reported in this paper we tested whether roughness perception shows a linear or an inverted $U$-shape relationship with inter-element spacing of dot patterns, and whether this relationship is different for haptic, visual or combined visual-haptic input. We found an effect of sensory modality on roughness perception. The relationship of inter-element spacing and roughness perceived by touch, by vision as well as by vision and touch 
combined was best described by a bi-exponential psychophysical function. Haptic and visual-haptic roughness perception peaked between 3 and $4 \mathrm{~mm}$ and were in the same order of magnitude. In contrast, visual roughness peaked already at about $1.25 \mathrm{~mm}$ inter-element spacing when tested in isolation, and this effect was invariant to differences in the scale context. Moreover, lower visual than haptic and bimodal roughness scores were found, primarily for textures with inter-dot spacings above $3 \mathrm{~mm}$. Furthermore, the order of modality conditions affected the visual roughness function in a setting in which subjects were asked to combine both visual and haptic information in a single bimodal judgment. There was a trend towards greater similarity between visual and haptic and visual-haptic roughness perception in terms of peak location when haptic preceded visual experience with the dot pattern textures. This influence of sensory familiarity on visual roughness scores was not replicated in a context where subjects were merely asked to perform unimodal visual and haptic roughness estimations. By comparing results obtained by using real and virtual visual textures, we were able to show that the main findings do not change in an experimental context with restricted viewing conditions.

In the first three experiments haptically perceived roughness was characterized by an increase up to an inter-dot spacing of about $3 \mathrm{~mm}$, decreasing again after a short plateau. We speculate that the number of indentations of the skin as well as the local spatial displacement (i.e., the depth of indentations) caused by the single dots influence the haptic roughness function. More specifically, for widely spaced textures the number of indentations is low while the local spatial displacement is maximal. If dots are added to the texture, the number of indentations will increase, thus increasing the perceived roughness. Below a certain dot distance, the local spatial displacement of the skin as well as the ability to resolve the single texture elements will be increasingly restricted by the skin's (lack of) compliance, thus the resulting roughness percept will decrease again.

In the first and third experiments we found attenuated roughness scores in the visual compared to the haptic and bimodal condition, which was not reported in a study conducted by Lederman and Abbott (1981) and which we also failed to find in our second study with a natural visual condition. There are several explanations for the difference in the results. One explanation might be the differences in presentation mode of the stimuli. Lederman and Abbott (1981) presented the textures in their natural form in the visual and bimodal condition, as we did in our second experiment, while we presented the textures on a monitor for visual exploration in experiments 1 and 3. This change of presentation mode between the haptic and visual condition in our experiments 1 and 3 might have induced or enhanced differences in roughness estimations between the conditions. However, the different findings in both studies can also be explained by the macrospatial structure of the textures that we used. The difference in perceived roughness magnitude between the visual condition and settings in which also haptic information was available is a logical finding for 
unfamiliar dot pattern stimuli when taking the results of experiments 3-5 into account. Our results suggest that visual and haptic roughness perception are both well described by a bi-exponential function of inter-dot spacing, shifted toward smaller spacings for visual exploration probably due to the higher visual spatial resolution. This shift results in attenuated visual roughness scores for textures above approximately $3 \mathrm{~mm}$ dot distance, textures that were overrepresented in our set of stimuli, and in higher visual roughness scores for textures with smaller inter-dot spacings. The interaction of modality and spacing was found in all 3 of our experiments containing haptic roughness estimation conditions. One explanation for the missing main effect of modality in the second experiment is the rather small effect of attenuated visual roughness scores for inter-element spacings above $3 \mathrm{~mm}$. This attenuation might have been cancelled out by the rather strong difference between visual and haptic roughness judgments for smaller inter-dot spacings. Finally, another explanation could be the influence of the task demands and haptic stimulus familiarity, which are found to affect visual roughness estimation strategies in the current study. The significant interaction of sensory modality, first condition and inter-dot distance in both experiments containing bimodal conditions indicates that the relationship of dot spacing and roughness estimation differs not only with the kind of sensory information available but it is also modulated by the kind of previous experience with the stimuli. An alternative explanation for the conflicting findings might therefore be a difference in the familiarity with the stimulus material. Lederman and Abbott (1981) used abrasive paper which is familiar to most people and therefore the visual judgments might have been already influenced by the haptic experience with those textures, diminishing the difference between visual and haptic magnitude estimates. Furthermore, subjects were allowed to practice for one day and therefore to become well acquainted with all stimuli. In contrast, in our experiment subjects had only few practice trials before each condition started. Hence, the factor haptic familiarity might have influenced roughness perception. This hypothesis is further supported by the results of the curve fitting procedure in the first and second experiment. The relationship of roughness perception and inter-dot spacing in experiments 1 and 2 is clearly influenced by the sensory information available for the judgments but also by previous experience with the stimulus material. When combined visual-haptic roughness judgments are required people appear to use preferably haptic imagery strategies to estimate visual roughness and therefore to calibrate the visual estimates to haptic roughness perception. In a setting in which extensive haptic experience with the stimulus material is available, this might result in a complete elimination of visual and haptic differences, which would be consistent with the findings from Lederman and Abbott (1981) for finer textures.

Several individuals reported that they used haptic imagery for visual roughness estimation when bimodal judgments were required and others utilized the high spatial resolution of the visual sense for roughness estimation 
when a combination of the unimodal information was not necessary. Still, some variation in the applied strategies and hence in the psychophysical roughness functions remained. This indicates an effect of individual biases toward the use of haptic or visual information in texture perception, as discussed by Guest and Spence (2003b) in the context of a multisensory task. Nevertheless, the results of our first and second experiments clearly indicate that bimodal roughness perception of unfamiliar dot pattern stimuli is more influenced by haptic than by visual information, in line with previous findings (Heller, 1982; Lederman et al., 1986). It is noteworthy, however, that there are also alternative explanations for the close resemblance of the bimodal and haptic roughness judgments in the work presented here. Although the experimenter monitored the performance of the participants in all conditions, it is possible that subjects focused their attention mainly on the haptic exploration in the bimodal condition and neglected the visual exploration of the textures. This would result necessarily in very similar haptic and bimodal roughness estimates. However, when asked to describe their rating strategies in the debriefing phase, none of our participants reported to have completely neglected the visual information in the visualhaptic condition. There is, therefore, every indication that subjects indeed performed the visual exploration as instructed. The use of an eyetracker system in future studies might be valuable for controlling the visual exploration behavior in the bimodal condition, although one can still not be certain whether the visual information is actually taken into account for bimodal judgments.

\section{CONCLUSIONS}

We reported the findings of a series of five experiments investigating the effect of sensory modality and familiarity on perceived roughness of unfamiliar textures. Visual, haptic and bimodal visual-haptic roughness perception of unfamiliar dot pattern stimuli is best characterized by a biexponential function of inter-dot spacing. However, the sensory modality used to explore the textures has an effect on the perceived magnitude of roughness. This is likely due to the difference in spatial resolution between the haptic and visual modality, resulting in a shift of the peak of the bi-exponential visual roughness function toward higher spatial resolution textures. Moreover, individuals appear to adapt their visual roughness estimation strategies based on the task demands, namely whether bimodal or only unimodal evaluations are required and on the sensory familiarity with the stimulus material. For the set of stimuli employed, a bias toward haptic information was demonstrated for bimodal visual-haptic roughness estimations. The overall results were independent of the presentation mode of the visual textures (virtual vs. real textures), indicating the suitability of the experimental design for neuroimaging studies with restricted viewing conditions and allowing to establish a more direct link between behavioral and neuroimaging results. 


\section{ACKNOWLEDGMENTS}

The research leading to these results has received funding from the European Community's Seventh Framework Programme FP7/2007-2013 under grant agreement number PITN-GA-2008-214728. Rainer Goebel and Amanda Kaas also gratefully acknowledge the support of the BrainGain Smart Mix Programme of the Netherlands Ministry of Economic Affairs and the Netherlands Ministry of Education, Culture and Science.

We would like to thank Ron Hellenbrand for his help in designing the stimulus material. We are also grateful to Minye Zhan for her assistance with the data acquisition and to Dr. Hong Z. Tan for the very helpful remarks on the results of the first experiment. 


\section{REFERENCES}

Bensmaia, S., Hollins, M., 2005. Pacinian representations of fine surface texture. Percept. Psychophys. 67, 842-854.

Bergmann Tiest, W.M., 2010. Tactual perception of material properties. Vision Res. 50, 2775-2782.

Bergmann Tiest, W.M., Kappers, A.M.L., 2007. Haptic and visual perception of roughness. Acta Psychol. (Amst) 124, 177-189.

Bergmann Tiest, W.M., Kappers, A.M.L., 2006. Analysis of haptic perception of materials by multidimensional scaling and physical measurements of roughness and compressibility. Acta Psychol. (Amst) 121, 1-20.

Blake, D.T., Hsiao, S.S., Johnson, K.O., 1997. Neural coding mechanisms in tactile pattern recognition: The relative contributions of slowly and rapidly adapting mechanoreceptors to perceived roughness. J. Neurosci. 17, 7480-7489.

Burnham, K.P., Anderson, D.R., 2004. Multimodel inference: Understanding AIC and BIC in model selection. Sociol. Methods Res. 33, 261-304.

Cascio, C.J., Sathian, K., 2001. Temporal cues contribute to tactile perception of roughness. J. Neurosci. 21, 5289-5296.

Connor, C.E., Hsiao, S.S., Phillips, J.R., Johnson, K.O., 1990. Tactile roughness: Neural codes that account for psychophysical magnitude estimates. J. Neurosci. 10, 3823-3836.

Dépeault, A., Meftah, E.-M., Chapman, C.E., 2009. Tactile perception of roughness: Raised-dot spacing, density and disposition. Exp. Brain. Res. 197, 235-244.

Gescheider, G.A., Bolanowski, S.J., Greenfield, T.C., Brunette, K.E., 2005. Perception of the tactile texture of raised-dot patterns: A multidimensional analysis. Somatosens. Mot. Res. 22, 127-140.

Guest, S., Catmur, C., Lloyd, D., Spence, C., 2002. Audiotactile interactions in roughness perception. Exp. Brain. Res. 146, 161-171.

Guest, S., Spence, C., 2003a. Tactile dominance in speeded discrimination of textures. Exp. Brain. Res. 150, 201-207.

Guest, S., Spence, C., 2003b. What role does multisensory integration play in the visuotactile perception of texture? International J. Psychophysiol. 50, 63-80.

Helbig, H.B., Ernst, M.O., 2007a. Knowledge about a common source can promote visual-haptic integration. Perception 36, 1523-1533.

Helbig, H.B., Ernst, M.O., 2007b. Optimal integration of shape information from vision and touch. Exp. Brain. Res. 179, 595-606.

Heller, M.A., 1989. Texture perception in sighted and blind observers. Percept. Psychophys. 45, 49-54.

Heller, M.A., 1982. Visual and tactual texture perception: Intersensory cooperation. Percept. Psychophys. 31, 339-344.

Ho, Y.-X., Landy, M.S., Maloney, L.T., 2006. How direction of illumination affects visually perceived surface roughness. J. Vis. 6, 634-648.

Ho, Y.-X., Maloney, L.T., Landy, M.S., 2007. The effect of viewpoint on perceived visual roughness. J. Vis., 7, 1-16.

Hollins, M., Risner, S.R., 2000. Evidence for the duplex theory of tactile texture perception. Percept. Psychophys. 62, 695-705.

Jones, B., O'Neil, S., 1985. Combining vision and touch in texture perception. Percept. Psychophys. 37, 66-72. 
Kahrimanovic, M., Bergmann Tiest, W.M., Kappers, A.M.L., 2009. Context effects in haptic perception of roughness. Exp. Brain. Res. 194, 287-297.

Katz, D., 1989. The world of touch. Hillsdale, England: Lawrence Erlbaum Associates, Inc (Original work published in 1925.).

Klatzky, R.L., Lederman, S.J., 1999. Tactile roughness perception with a rigid link interposed between skin surface. Percept. Psychophys. 61, 591-607.

Klatzky, R.L., Lederman, S.J., Reed, C., 1987. There's more to touch than meets the eye: The salience of object attributes for haptics with and without vision. J. Exp. Psychol. Gen. 116, 356-369.

Lacey, S., Hall, J., Sathian, K., 2010. Are surface properties integrated into visuohaptic object representations? Eur. J. Neurosci. 31, 1882-1888.

Lawrence, M.A., Kitada, R., Klatzky, R.L., Lederman, S.J., 2007. Haptic roughness perception of linear gratings via bare finger or rigid probe. Perception 36,547 557.

Lederman, S.J., Abbott, S.G., 1981. Texture perception: Studies of intersensory organization using a discrepancy paradigm, and visual versus tactual psychophysics. J. Exp. Psychol. Hum. Percept. Perform. 7, 902-915.

Lederman, S.J., Klatzky, R.L., 2009. Haptic perception: A tutorial. Atten. Percept. Psychophys. 71, 1439-1459.

Lederman, S.J., Klatzky, R.L., Hamilton, C.L., Ramsay, G.I., 1999. Perceiving roughness via a rigid probe: Psychophysical effects of exploration speed and mode of touch. Haptics-e.

Lederman, S.J., Thorne, G., Jones, B., 1986. Perception of texture by vision and touch: Multidimensionality and intersensory integration. J. Exp. Psychol. Hum. Percept. Perform. 12, 169-180.

Legge, G.E., Madison, C., Vaughn, B.N., Cheong, A.M.Y., Miller, J.C., 2008. Retention of high tactile acuity throughout the life span in blindness. Percept. Psychophys. 70, 1471-1488.

Meftah, E.-M., Belingard, L., Chapman, C.E., 2000. Relative effects of the spatial and temporal characteristics of scanned surfaces on human perception of tactile roughness using passive touch. Exp. Brain. Res. 132, 351-361.

Merabet, L.B., Swisher, J.D., McMains, S.A., Halko, M.A., Amedi, A., Pascual-Leone, A., Somers, D.C., 2007. Combined activation and deactivation of visual cortex during tactile sensory processing. J. Neurophysiol. 97, 1633-1641.

Merabet, L.B., Thut, G., Murray, B., Andrews, J., Hsiao, S., Pascual-Leone, A., 2004. Feeling by sight or seeing by touch? Neuron, 42, 173-179.

Sekuler, R., Blake, R., 2001. Perception (4th ed.). New York: McGraw-Hill.

Wagenmakers, E.-J., Farrell, S., 2004. AIC model selection using Akaike weights. Psychon. Bull. Rev. 11, 192-196.

Yoshioka, T., Bensmaia, S.J., Craig, J.C., Hsiao, S.S., 2007. Texture perception through direct and indirect touch: An analysis of perceptual space for tactile textures in two modes of exploration. Somatosens. Mot. Res. 24, 53-70. 


\section{Chapter 3}

\section{Crossmodal interactions of haptic and visual texture information in early sensory cortex}

Based on: Eck, J., Kaas, A.L., Goebel, R., 2013. Crossmodal interactions of haptic and visual texture information in early sensory cortex. Neuroimage 75, 123-135. 


\section{ABSTRACT}

Both visual and haptic information add to the perception of surface texture. While prior studies have reported crossmodal interactions of both sensory modalities at the behavioral level, neuroimaging studies primarily investigated texture perception in separate visual and haptic paradigms. These experimental designs, however, only allowed to identify overlap in both sensory processing streams but no interaction of visual and haptic texture processing. By varying texture characteristics in a bimodal task, the current study investigated how these crossmodal interactions are reflected at the cortical level. We used fMRI to compare cortical activation in response to matching versus non-matching visual-haptic texture information. We expected that passive simultaneous presentation of matching visual-haptic input would be sufficient to induce BOLD responses graded with varying texture characteristics. Since no cognitive evaluation of the stimuli was required, we expected to find changes primarily at a rather early processing stage. Our results confirmed our assumptions by showing crossmodal interactions of visual-haptic texture information in early somatosensory and visual cortex. However, the nature of the crossmodal effects was slightly different in both sensory cortices. In early visual cortex, matching visual-haptic information increased the average activation level and induced parametric BOLD signal variations with varying texture characteristics. In early somatosensory cortex only the latter was true. These results challenge the notion that visual and haptic texture information is processed independently and indicate a crossmodal interaction of sensory information already at an early cortical processing stage. 


\section{INTRODUCTION}

Humans need to be able to differentiate surface qualities of objects not only by touch but also visually. This is important for object recognition (e.g. nectarine vs. peach) and for the interaction with objects in our environment (Fikes et al., 1994) (e.g. for goal-directed movement: grasping a slippery piece of soap vs. a splintering piece of wood). Behavioral studies showed that both haptic and visual informations add to texture perception (Lederman and Abbott, 1981) and that a crossmodal transfer of texture information between both sensory modalities occurs (Picard, 2006).

However, it is only in the last decade that the neural basis of texture perception and its multidimensional experience have received increased attention. Several neuroimaging studies focused on texture matching and discrimination (Cant and Goodale, 2007; Cavina-Pratesi et al., 2010a; Kaas et al., 2013; Peuskens et al., 2004; Sathian et al., 2011; Stilla and Sathian, 2008) as well as on different dimensions of texture perception within the tactile and visual modality; examples include spatial density (Merabet et al., 2004; Zhang et al., 2005), spatial orientation (Kitada et al., 2006; Sathian et al., 1997; Zhang et al., 2005) and roughness (Burton et al., 2008; Merabet et al., 2004; Kitada et al., 2005; Roland et al., 1998; Simões-Franklin et al., 2011). Most of the tactile studies stress the importance of the posterior insula and the parietal operculum (OP1, OP3 and OP4) (Kaas et al., 2013; Kitada et al., 2005; Roland et al., 1998; Simões-Franklin et al., 2011; Stilla and Sathian, 2008) for processing surface textures, while studies focusing on visual texture perception often report regions near the collateral sulcus, the lingual gyrus and areas in early visual cortex (Cant and Goodale, 2007, 2011; Cant and Xu, 2012; Cavina-Pratesi et al., 2010b; Peuskens et al., 2004; Stilla and Sathian, 2008; Sathian et al., 2011).

Next to the identification of cortical key players in visual and haptic texture perception a recent approach by Hiramatsu et al. (2011) aimed at investigating how visual material properties are coded in the cortex along the ventral visual pathway. The authors reported that while both early and higherorder visual areas seem to contain information distinguishing material categories (including texture information), the neural representation shifts gradually from an image-based representation in early areas (V1/V2 and $\mathrm{V} 3 / \mathrm{V} 4)$ to a perceptual representation in areas around the fusiform gyrus and the collateral sulcus. Hence, physical and perceptual measures of visual material qualities seem to be processed in a spatially distributed network in the visual cortex, rather than in a single localized region. A similar distributed network was described by Sathian et al. (2011) for the processing of haptic texture information. In connectivity analyses Sathian and colleagues showed a flow of texture information from task-non-selective regions of the postcentral gyrus to texture-selective areas in the parietal operculum (OP3) and further to regions of the middle occipital cortex. Despite the pure tactile stimulation in many paradigms, consistent visual cortex activation was reported in several of these 
studies (Merabet et al., 2007; Simões-Franklin et al., 2011; Stilla and Sathian, 2008). Some findings even indicate the existence of bisensory texture-selective regions in the posterior visual cortex and the lingual gyrus by comparing activations elicited by unimodal shape, location and texture matching in both the visual and haptic modality (Sathian et al., 2011; Stilla and Sathian, 2008).

All of the above-mentioned studies investigated texture perception in separate visual and haptic paradigms. The effect of simultaneous visual and haptic exploration of textures has been mostly neglected so far. Hence, we can only assume an overlap of visual and haptic texture representations in some brain areas, but we cannot infer from these studies whether visual and haptic information interacts in these cortical regions. The imaging study by Sathian et al. (2011) gives a first indication that this might indeed be the case. Behavioral studies also indicate the existence of such crossmodal interaction and matching effects in visuo-haptic tasks. It was shown that people consistently and absolutely match specific tactile vibration rates (simulating manual exploration of a textured surface) to visual spatial frequencies (Guzman-Martinez et al., 2012), indicating some kind of crossmodal association effect in visual and haptic texture perception. Furthermore, Lunghi et al. (2010) even showed that simultaneous tactile stimulation can disambiguate binocular rivalry, a process in which two equally salient but dissimilar monocular stimuli are presented to corresponding retinal locations. Both stimuli compete for perceptual dominance and at any instant only one is perceived consciously while the other image is suppressed. In this study subjects haptically explored a linear grating with a matching orientation to either one of two rival visual stimuli. Exploration of the haptic stimulus prolonged dominance or reduced suppression of the matching visual stimulus, indicating a crossmodal interaction. The authors infer from these results that haptic information can modulate visual processing already at a very early stage, probably in V1. This raises the question whether a change in cortical processing can be expected when matching as compared to nonmatching visual-haptic texture information is provided, i.e. representing crossmodal interactions at the cortical level.

In a unimodal tactile fMRI study, Kitada et al. (2005) used a parametric stimulus set, i.e. linear gratings varying in spatial period, and demonstrated that differences in tactile roughness yield graded BOLD responses in the parietal operculum, insula and the lateral prefrontal cortex, but only when subjects actively judge rather than merely attend to roughness. Assuming that crossmodal interactions of texture information are not only presented at the behavioral but also at the cortical level, the question arises whether matching visual-haptic information is sufficient for the observation of graded BOLD responses with varying texture characteristics even without an active judgment task.

The main objective of the present study was to investigate texture perception in a paradigm that combines visual and haptic input in a single condition in order to explore crossmodal interactions at the cortical level. We 
propose differences in cortical processing of matching and non-matching visual-haptic texture information, representing the influence of one sensory modality on information processing in the other modality as indicated by earlier behavioral studies. Based on the studies mentioned above we would expect these crossmodal effects already in early sensory cortices, e.g. postcentral gyrus and posterior occipital cortex (Dionne et al., 2010; Hiramatsu et al., 2011; Merabet et al., 2007; Sathian et al., 2011; Stilla and Sathian, 2008), but perception-related differences rather in higher-order cortical regions, e.g. the collateral sulcus as well as the parietal operculum and the insula (Cavina-Pratesi et al., 2010b; Hiramatsu et al., 2011; Kitada et al., 2005). The unfamiliar dot pattern textures used in this experiment varied only along a single texture dimension, i.e. the average center-to-center dot spacing, ensuring that changes in other surface properties like color and friction do not influence the results. Stimulus presentation was always bimodal, but the sensory information content differed as texture information was varied either in the haptic, visual or in both channels.

We analyzed the data in two different ways. First we were interested in the average difference of the BOLD signal between both unimodal and the bimodal texture variation conditions, disregarding dot pattern differences. As we did not ask subjects to perform a cognitive task with the presented textures, we would expect to find differences, if any, at a rather early sensory processing stage. Second we were interested in relative differences of the BOLD response within each of these three conditions, taking into account the parametric dot pattern variation. Is the BOLD response modulated by texture differences when texture characteristics are varied either unimodally or bimodally? Based on previous behavioral studies we expected perceived roughness by touch to be almost perfectly correlated with the inter-dot spacing (Connor et al., 1990; Dépeault et al., 2009; Eck et al., 2011), while visual spatial density estimates should be negatively correlated with average inter-dot distance. Hence, no difference in the parametric BOLD modulation was expected between the two subjective measures tactile roughness and visual spatial density and the objective texture characteristic inter-dot spacing. However, to account for possible subjective perceptual differences we used individual post-fMRI ratings of haptic roughness and visual spatial density as well as the physical inter-dot spacing of the textures in separate parametric models.

\section{METHOD}

\section{Participants}

Seventeen right-handed, healthy volunteers ( 13 women, 4 men; $27 \pm 5.9$ years) with normal or corrected-to-normal vision participated in the study. Subjects with calluses or injuries to the hands were excluded from participation. 
All participants were graduate and undergraduate students at Maastricht University. They were naïve to the hypotheses and received course credit or monetary compensation for their participation in the experiment. In accordance with the Declaration of Helsinki, written informed consent was obtained from each participant and the study was approved by the local ethics committee.

\section{Stimuli}

Haptic stimuli consisted of seven $5 \times 5 \mathrm{~cm}^{2}$ plastic plates, six embossed with different dot patterns and one control stimulus without any dots. The dots were arranged non-periodically and were $0.8 \mathrm{~mm}$ in diameter and $0.6 \mathrm{~mm}$ in elevation. The only characteristic that varied between the textures was the mean center-to-center dot spacing of each stimulus and hence the number of texture elements (dots). The average inter-dot spacing ranged from $1.50 \mathrm{~mm}$ to 2.75 $\mathrm{mm}$ and increased in steps of $0.25 \mathrm{~mm}$ (see Table 1 for detailed information on the stimulus characteristics). Details about the algorithm used to produce these textures can be found in Eck et al. (2011). For each dot matrix a 3D wireframe model was created and computer-rendered in AutoCAD® 2010 (Autodesk Inc., San Rafael, CA, USA) in order to create a set of matching visual stimuli. Two distant light sources following the direction of the viewpoint provided the lighting of each stimulus in such a way that all faces of the model were illuminated (see Figure 1).
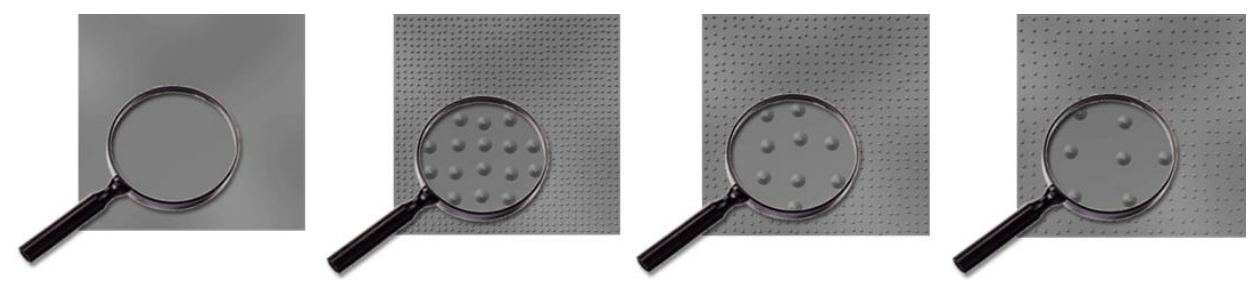

Figure 1. Examples of textures used in the experiment. The texture on the left shows the control stimulus without any dots. The other three textures are presented in order of increasing mean inter-dot spacing, from left to right: $1.50 \mathrm{~mm}, 2.00 \mathrm{~mm}$, and $2.50 \mathrm{~mm}$ (corresponding to stimuli 1, 3 and 5 in Table 1). 
Table 1 Texture characteristics of stimuli. Surface area of the stimuli $=25 \mathrm{~cm}^{2}$.

\begin{tabular}{llllllll}
\hline Stimulus & $\begin{array}{l}\text { Smooth } \\
\text { Control }\end{array}$ & 1 & 2 & 3 & 4 & 5 & 6 \\
\hline $\begin{array}{l}\text { Average Inter- } \\
\begin{array}{l}\text { Dot Spacing } \\
\text { in mm }\end{array}\end{array}$ & 1.50 & 1.75 & 2.00 & 2.25 & 2.50 & 2.75 \\
$\begin{array}{l}\text { Number Tex- } \\
\text { ture Elements }\end{array}$ & - & 1089 & 785 & 625 & 484 & 400 & 324 \\
\hline
\end{tabular}

\section{Experimental setup}

All textures were arranged on a circular wooden board, which was covered by a second wooden plate with a rectangular cut out. The cover-plate allowed subjects to touch only one texture at a time and served as the resting position for the hand in between exploration trials. This haptic texture presentation device was placed over the thighs of the participant and was attached to the scanner table. The right hand of the subject was placed on top of the cover-plate and the arm was supported by foam padding to reduce movement of the elbow and shoulder. The experimenter who was standing next to the scanner bore during the functional scans (see Figure 2) controlled the presentation of the haptic stimuli. Visual stimuli were displayed centrally on a black background and subtended 5.7 degrees of visual angle. The images were projected onto a rear-projection screen at the end of the scanner bore and subjects viewed the stimuli via a mirror mounted to the head-coil.

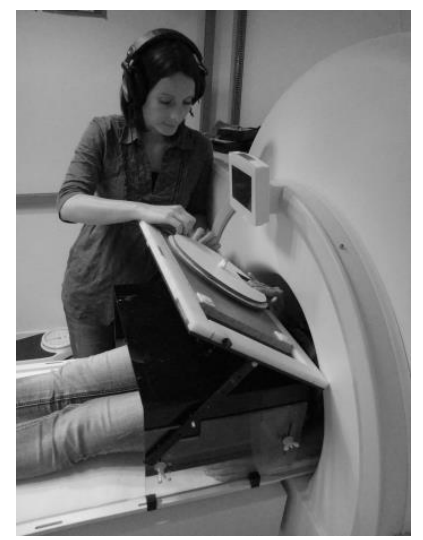

Figure 2. Presentation of the experimental setup. In the functional runs the experimenter presented the haptic stimuli to the participants. Auditory cues delivered via headphones to the experimenter controlled the stimulus timing of the haptic presentation. 


\section{Experimental procedure}

Stimulus timing and presentation was controlled by Presentation ${ }^{\circledR}$ software (Neurobehavioral Systems, Inc., Albany, CA, USA). Subjects were informed that they would be presented with one visual and one haptic texture at each trial. Participants were instructed to explore the textures by sweeping twice with their right index-, middle- and ring fingers across the surface while simultaneously focusing on the visual image presented on the screen. The importance of the simultaneous start and termination of the visual and haptic exploration was specifically stressed by the experimenter in order to control the temporal synchrony of the sensory input. Trial intervals were intermixed with intervals of rest. Auditory cues delivered via headphones instructed the experimenter to turn the haptic presentation device to the correct stimulus during the inter-stimulus-intervals. Right before scanning started subjects practiced the exploration movement in the scanner with two dot pattern textures that were not used in the experiment. These textures had an average inter-dot spacing of 7.00 and $7.50 \mathrm{~mm}$ respectively. The practice session lasted until the movement was experienced by the subject as effortless, it was synchronized with the duration of the exploration interval and all other motion was reduced to a minimum. This took on average five minutes but never longer than ten minutes. The practice session ensured that the attention of the subject was not focused on the motion sequence but on the tactile and visual sensations.

There was a visual, haptic, and visual-haptic condition in which the availability of texture information for the tactile and visual sense was varied but the input modalities and motor task demands were kept constant. Thus, subjects always explored the stimuli both visually and haptically, but in the haptic condition a smooth control stimulus without any texture information was presented visually (unimodal haptic dot pattern condition) and in the visual condition vice versa (unimodal visual dot pattern condition). In the visualhaptic condition matching dot patterns were presented visually and haptically (bimodal dot pattern condition). Each dot pattern was repeated 19 times in each condition over the course of the experiment. A fast event-related design was utilized, consisting of alternating exploration periods ( $2 \mathrm{~s})$ and inter-stimulus intervals $(4 \mathrm{~s})$ in which a fixation cross was displayed (see Figure 3 ). The condition and stimulus presentation was semi-randomized and the 342 events of interest were randomly interspersed with 108 null events. The experiment was split into three functional runs. The event-related fMRI design was based on an approach described by Kao et al. (2009).

After the scanning session, subjects assessed the perceived haptic roughness and visual spatial density of each stimulus on a 10-point rating scale ranging from 1 (very smooth/sparse) to 10 (very rough/dense). In order to avoid conscious rating of the stimuli during the fMRI experiment participants were not aware that they would be asked to assess these texture characteristics at a later time point. 


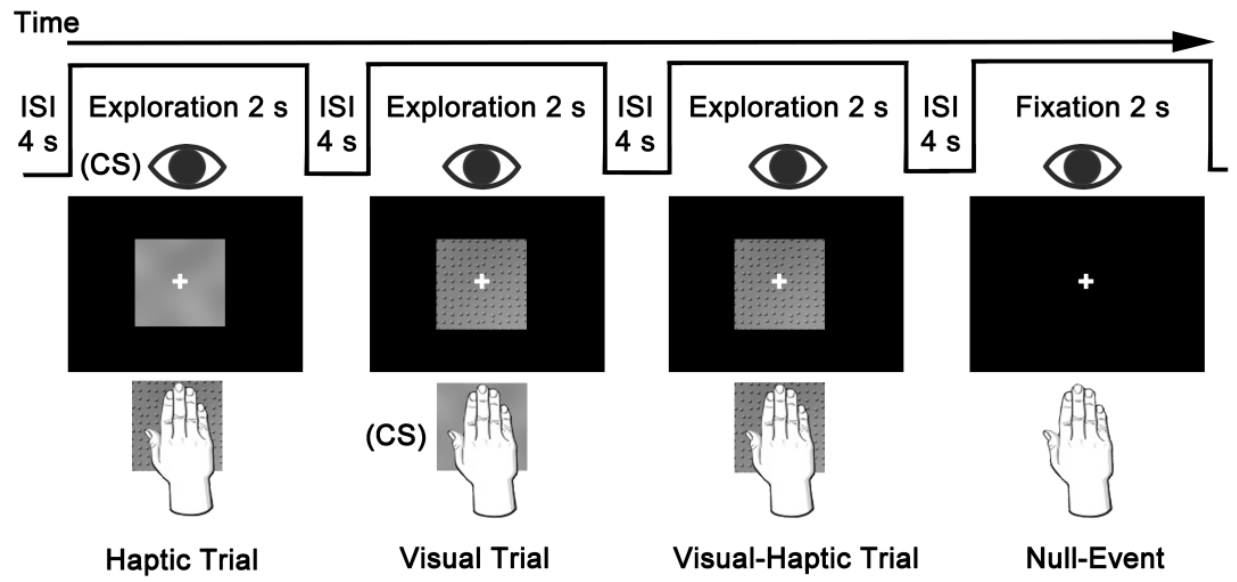

Figure 3. Schematic description of all experimental trials. During the inter-stimulus interval a fixation cross was presented. $\mathrm{CS}=$ control stimulus; ISI $=$ inter-stimulus interval.

\section{Data acquisition}

Data were acquired using a 3 Tesla MRI head scanner (Siemens Allegra, Erlangen, Germany) at the Maastricht Brain Imaging Center (Maastricht, The Netherlands). Subjects were placed comfortably in the scanner and foam padding around the head was used to minimize head movement. Functional images were obtained with a $\mathrm{T} 2 *$-weighted echo-planar imaging (EPI) sequence ( scan parameters: $\mathrm{TR}=2000 \mathrm{~ms}, \mathrm{TE}=30 \mathrm{~ms}$, flip angle $(\mathrm{FA})=$ $90^{\circ}$, matrix $=64 \times 64$, field of view $(\mathrm{FOV})=224 \times 224$, slice thickness $=3.5$ $\mathrm{mm}, 32$ slices, no gap) and comprised 458 volumes for each run. In between the second and third functional runs a standard 3D high-resolution data set (MPRAGE) covering the whole brain was acquired (scan parameters: TR = $2250 \mathrm{~ms}, \mathrm{TE}=2.6 \mathrm{~ms}, \mathrm{FA}=9^{\circ}$, matrix: $256 \times 256$, voxel size: $1 \times 1 \times 1 \mathrm{~mm}^{3}$, 192 slices).

\section{Data analysis}

Post-scanning roughness estimates and spatial density scores of all subjects were analyzed with SPSS 18 (SPSS Inc., Chicago, IL, USA).

Functional and anatomical data were analyzed with BrainVoyager QX 2.3.1 (Brain Innovation, Maastricht, The Netherlands). The first 2 scans of each functional run were discarded from analysis to eliminate T1 saturation effects. Preprocessing of the functional data included the following steps: slice scan time correction, intra-session alignment to detect and correct for small head 
movements by rigid body transformations, temporal filtering removing linear trends and non-linear temporal frequencies of 3 or less cycles per run and spatial smoothing with an isotropic Gaussian kernel of $6 \mathrm{~mm}$ FWHM. None of the participants moved more than $2.9 \mathrm{~mm} /$ degrees in any direction. Functional images of all runs were co-registered to the anatomical volumes of the respective subject and transformed into Talairach space resulting in an interpolated functional voxel size of $3 \times 3 \times 3 \mathrm{~mm}^{3}$.

For group analysis a two-level general linear model (GLM) approach was used. At single-subject level a whole-brain fixed-effects regression analysis was performed with four different models, each addressing a different aspect of the research questions.

Differences in the BOLD response between unimodal and bimodal dot pattern conditions

Model 1 Comparison of modality effects. For each subject a set of three task-related regressors was defined, one for each modality condition (visual dot pattern condition $-\mathrm{V}$, haptic dot pattern condition $-\mathrm{H}$, visual-haptic dot pattern condition - VH), irrespective of the explored dot pattern. Furthermore, seven z-transformed confound regressors were added, including a predictor for the instruction interval in the beginning of each run and the six motion parameters to account for residual motion artifacts in the time courses.

For visualization of the estimated hemodynamic response function (HRF) of all modality conditions, we used a deconvolution analysis. This was necessary because BOLD responses to individual stimuli substantially overlap in rapid event-related designs, which renders the raw signal useless for visualization of mean signal changes between conditions. A set of ten consecutive stick predictors of 1 TR was defined for each modality condition in order to cover the temporal extent of the hemodynamic response (20 s). Beta weights of these stick functions were used to plot the shape of the estimated HRF of all modality conditions.

Differences related to parametric dot pattern variation within each modality condition

Model 2 Test for modulation of the BOLD response by objective texture characteristics (mean inter-dot spacing). The regression model consisted of a set of three main predictors for all modality conditions (Vmain, Hmain, VHmain) and three predictors coding for the parametric modulation of the HRF by the mean inter-dot spacing of the stimuli in the three modality conditions (Vpara, Hpara, VHpara). Additionally, we included the same confound predictors as in model 1. All predictors of this model were z-transformed. 
Model 3 Test for modulation of the BOLD response by haptically perceived roughness. Model 3 was identical to model 2, but for the parametric predictors the haptically estimated roughness of the stimuli was used.

Model 4 Test for modulation of the BOLD response by visually perceived spatial density. This model was also identical to model 2 , but this time visually perceived spatial density was used for the parametric predictors in the three modality conditions.

In order to visualize the mean BOLD response of each dot pattern texture in all three modality conditions, a deconvolution analysis was computed. A set of ten consecutive stick predictors of $1 \mathrm{TR}$, covering a temporal interval of $20 \mathrm{~s}$, was defined for each texture in all three modality conditions. Beta weights of these stick functions were used to plot the shape of the estimated HRF for all dot pattern textures in different cortical regions.

All predictor time courses in models $1-4$ with the exception of the motion parameters were convolved with a 2-gamma HRF.

At group level, whole-brain random-effects analyses were performed for all models defined at the single subject level. A statistical model of the main modulation effect of sensory modality (model 1 ) was fitted to the data to reveal brain regions that respond differently depending on the texture information accessible to the visual and haptic modality. We were interested in the difference between the visual-haptic $(\mathrm{VH})$ and both visual $(\mathrm{V})$ and haptic $(\mathrm{H})$ dot pattern conditions $([\mathrm{VH}>\mathrm{V}],[\mathrm{VH}>\mathrm{H}])$ as well as in the difference between both unimodal information variation conditions $(\mathrm{V} \neq \mathrm{H})$. Furthermore, in order to reveal brain regions that show an enhanced response when texture information varies bimodally compared to either unimodal variation of information, we computed the following conjunction contrast: $[\mathrm{V}] \cap[\mathrm{H}] \cap[\mathrm{VH}$ $>\mathrm{V}] \cap[\mathrm{VH}>\mathrm{H}]$.

Additionally, models related to the parametric texture modulation (models 2-4) were fitted to the data to explore cortical areas that differ in their response with respect to objective texture characteristics (mean inter-dot spacing) or subjectively perceived differences between textures (haptically perceived roughness and visually perceived spatial density). The specificity of the result was provided by the conjunction contrast of the main and parametric predictor for each modality condition ([Vmain] $\cap$ [Vpara], [Hmain] $\cap$ [Hpara], [VHmain] $\cap$ [VHpara]), requiring both a significant main effect of modality and a significant parametric modulation of the HRF in the same cortical location.

The voxel-threshold for statistical significance was first set to $\alpha=0.005$ uncorrected and all statistical contrast maps were then corrected for multiple comparisons using cluster-size thresholding (Forman et al., 1995; Goebel et al., 2006) with a cluster-level false positive rate of $\alpha=0.05$. 


\section{RESULTS}

\section{Behavioral results}

In general perceived haptic roughness increased with increasing inter-dot distance while visually perceived spatial density decreased (see Figure 4). This was expected from earlier work conducted by our group (Eck et al., 2011) and it was confirmed by a significant linear effect of spacing in two repeated measures ANOVAs of spacing on perceived roughness by touch and visual spatial density $\left(\mathrm{F}(1,16)=174.82, \mathrm{p}<0.001\right.$, partial $\eta^{2}=0.92$ and $\mathrm{F}(1,16)=167.8$, $\mathrm{p}<0.001$, partial $\eta^{2}=0.91$, respectively). Bonferroni-corrected post-hoc tests showed significant differences between all levels of spacing for roughness perceived by touch as well as for spatial density perceived by vision.

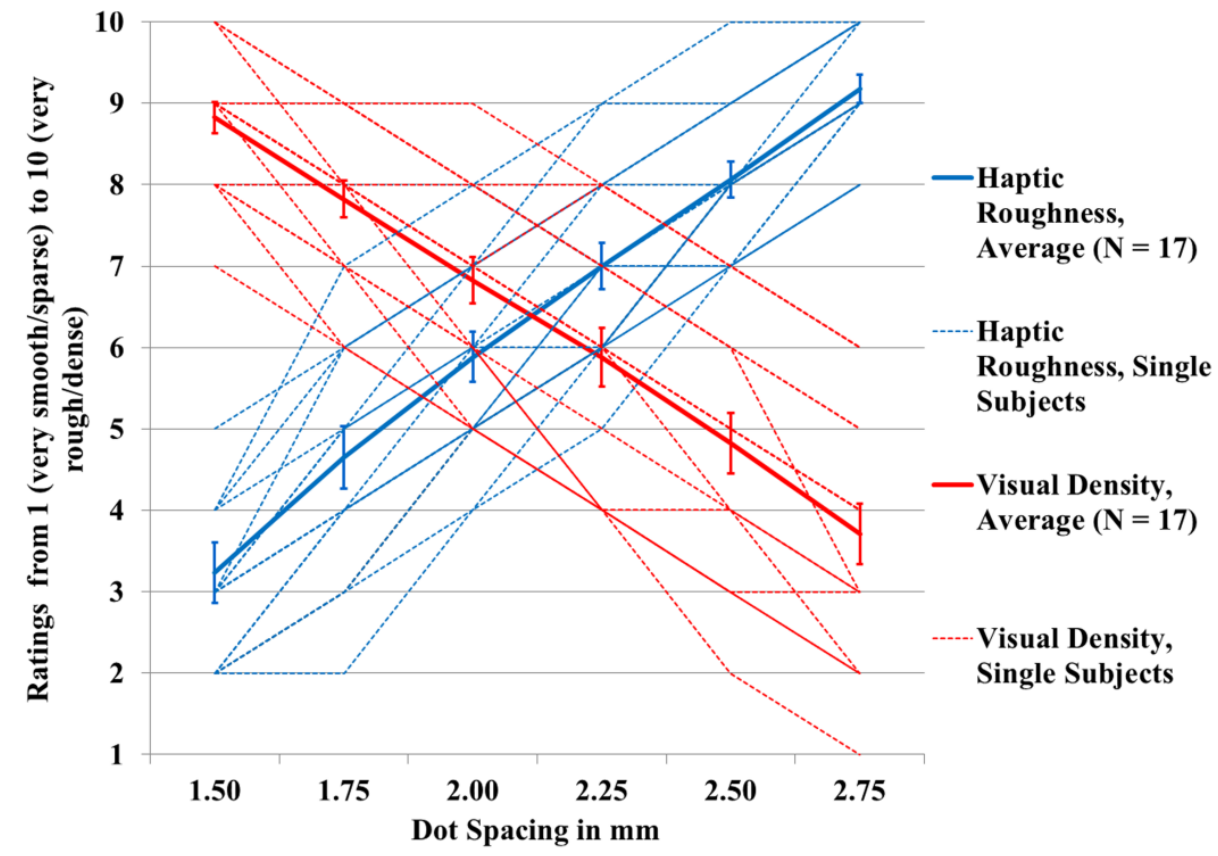

Figure 4. Visual spatial density and haptic roughness judgments of all dot pattern textures. Roughness and density ratings of each participant and averaged over all subjects. Bars represent the standard error of the mean (SEM).

The average correlation of inter-dot spacing with perceived roughness and spatial density across subjects was 0.90 and -0.97 respectively. The correlation of both subjective texture characteristics was -0.93 across subjects. 


\section{fMRI results}

\section{Model 1 Differences in the BOLD response between unimodal and bimodal dot pattern conditions}

$[V] \cap[H] \cap[V H]$. As presented in Figure 5, texture exploration activated a cortical network involving early and higher-order visual, motor and somatosensory regions as well as prefrontal and cingulate areas. The consistency of this network across the different modality conditions is considerable as would have been expected by the constant sensory task demands across modality conditions.

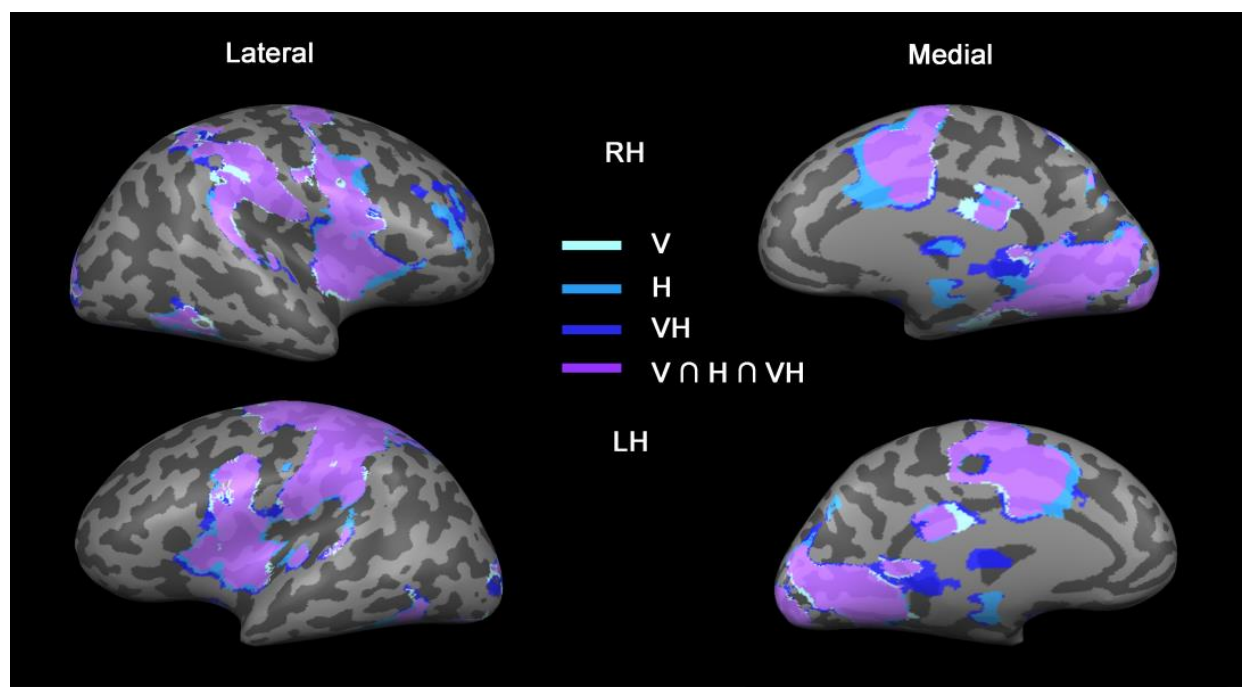

Figure 5. Overlap of modality maps. Random effect maps of each modality condition versus baseline and the conjunction of these three maps displayed on an inflated surface reconstruction of a single representative subject; $\mathrm{V}=$ dot pattern information in visual modality (haptic control stimulus); $\mathrm{H}=$ dot pattern information in haptic modality (visual control stimulus); $\mathrm{VH}=$ matching dot pattern information in visual and haptic modality; $\mathrm{p}<0.05$ corrected at cluster level; $\mathrm{LH}=$ left hemisphere, $\mathrm{RH}=$ right hemisphere.

Despite the spatial consistency of the network involved in texture perception, there are noticeable differences in response to the presence of dot patterns in the visual or haptic modality only versus congruently in both sensory modalities. These differences are summarized in Figure 6 and Table 2. 
Table 2. Differences in the BOLD response between unimodal and bimodal dot pattern conditions (model 1).

\begin{tabular}{|c|c|c|c|c|c|c|}
\hline Contrast & Region & $\begin{array}{l}\text { Hemi- } \\
\text { sphere }\end{array}$ & $\mathrm{x}$ & $\mathrm{y}$ & $\mathrm{z}$ & $\mathrm{t}$ \\
\hline \multicolumn{7}{|l|}{$[\mathrm{V}>\mathrm{H}]$} \\
\hline & pCS, lateral pOCC, LG & $\mathrm{R}$ & 17 & -89 & -9 & 4.80 \\
\hline & pCS, lateral pOCC & $\mathrm{L}$ & -13 & -98 & -12 & 4.29 \\
\hline & PoCG & $\mathrm{L}$ & -46 & -23 & 48 & -4.30 \\
\hline \multicolumn{7}{|l|}{$[\mathrm{VH}>\mathrm{H}]$} \\
\hline & pCS, lateral pOCC, LG & $\mathrm{L}$ & -16 & -95 & -6 & 5.77 \\
\hline & pCS, lateral pOCC, LG & $\mathrm{R}$ & 14 & -89 & -12 & 5.37 \\
\hline \multicolumn{7}{|c|}{ Pen, таштан pece, Lu } \\
\hline & $\mathrm{MeFG}$ & $\mathrm{L}$ & -10 & 13 & 45 & 4.79 \\
\hline & PreCG, PoCG, SMG & $\mathrm{L}$ & -37 & -35 & 66 & 8.04 \\
\hline & $\mathrm{pCS}$, lateral pOCC & $\mathrm{L}$ & -25 & -95 & -6 & 5.47 \\
\hline & Basal ganglia, thalamus & $\mathrm{L}$ & -22 & -2 & 6 & 5.57 \\
\hline & Basal ganglia & $\mathrm{R}$ & 8 & 7 & 9 & 4.66 \\
\hline & FG and cerebellum & $\mathrm{L}$ & -40 & -68 & -24 & 4.71 \\
\hline & Cerebellum & $\mathrm{R} / \mathrm{L}$ & 8 & -47 & -21 & 5.82 \\
\hline \multicolumn{7}{|c|}{$[\mathrm{V}] \cap[\mathrm{H}] \cap[\mathrm{VH}>\mathrm{V}] \cap[\mathrm{VH}>\mathrm{H}]$} \\
\hline & pCS, lateral pOCC & $\mathrm{L}$ & -19 & -95 & -6 & 4.29 \\
\hline
\end{tabular}

Note. $\mathrm{V}=$ dot pattern information in visual modality (haptic control stimulus); $\mathrm{H}=\mathrm{dot}$ pattern information in haptic modality (visual control stimulus); $\mathrm{VH}=$ matching dot pattern information in visual and haptic modality; pCS = posterior calcarine sulcus; pOCC $=$ posterior occipital cortex $; \mathrm{LG}=$ lingual gyrus; $\mathrm{PoCG}=$ postcentral gyrus; $\mathrm{MeFG}=$ medial frontal gyrus; PreCG = precentral gyrus; $\mathrm{SMG}=$ supramarginal gyrus; $\mathrm{FG}=$ fusiform gyrus; $\mathrm{x}, \mathrm{y}, \mathrm{z}=$ Talairach coordinates of peak voxel; $\mathrm{t}=$ peak $\mathrm{t}$ value; $\mathrm{R}=$ right hemisphere; $\mathrm{L}=$ left hemisphere; $\mathrm{p}<0.05$ corrected at cluster level.

$[V>H]$. The contrast of the visual and haptic dot pattern condition identified a region in both hemispheres of the early visual cortex that showed increased activation in the visual condition. This region was located in the posterior part of the calcarine sulcus (pCS) extending to the lateral part of the posterior occipital cortex (pOCC) and the right lingual gyrus (LG).

$[H>V]$. The opposite was true for the left postcentral gyrus (PoCG) which responded stronger in the haptic than in the visual condition. 


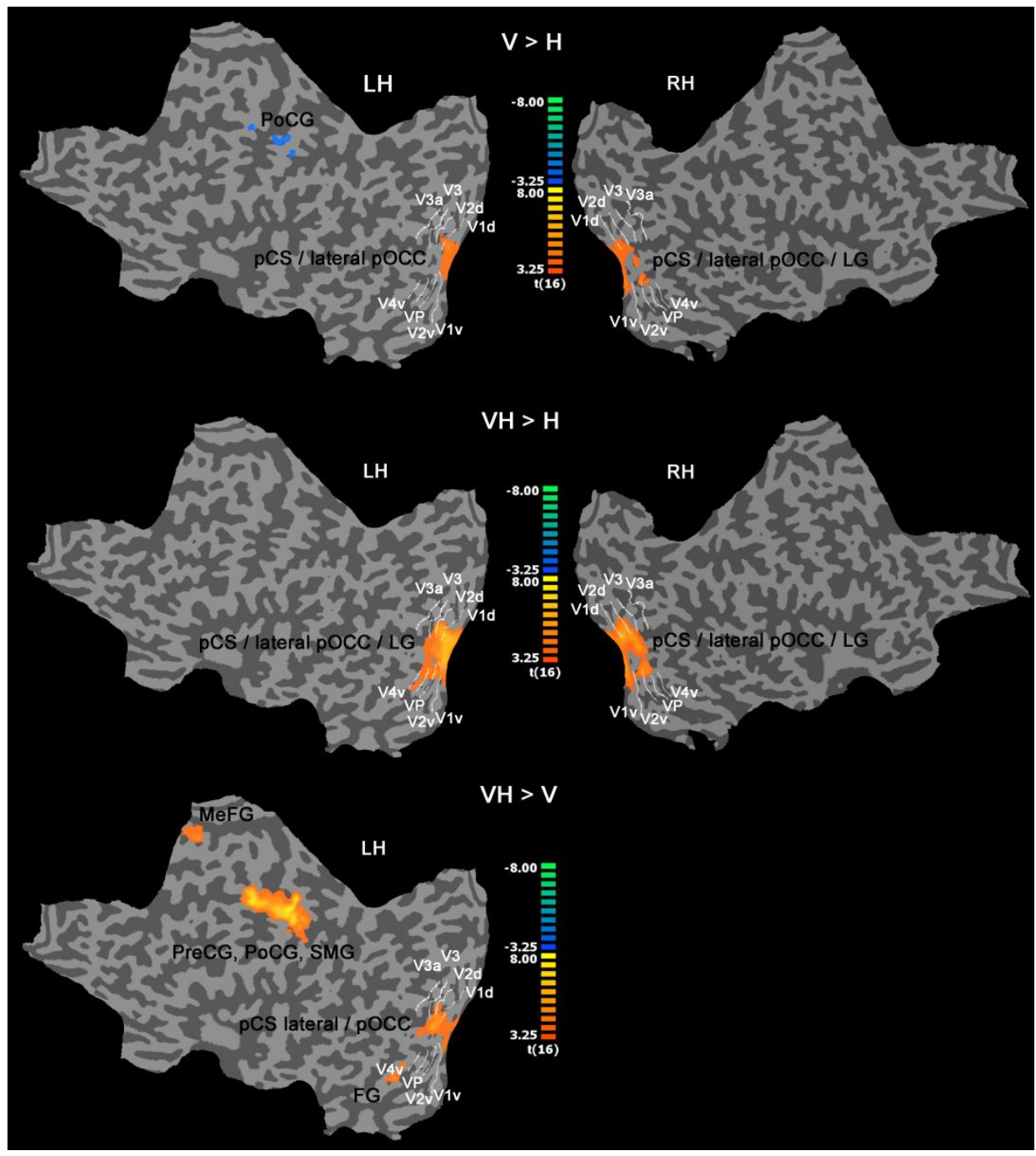

Figure 6. Differences in the BOLD response between unimodal and bimodal dot pattern conditions. Random effect maps are displayed on a flattened surface reconstruction of a single representative subject for which early visual areas were delineated by retinotopic mapping; $\mathrm{V}=$ dot pattern information in visual modality (haptic control stimulus); $\mathrm{H}=$ dot pattern information in haptic modality (visual control stimulus); $\mathrm{VH}=$ matching dot pattern information in visual and haptic modality; $\mathrm{PoCG}=$ postcentral gyrus; $\mathrm{pCS}=$ posterior calcarine sulcus; pOCC = posterior occipital cortex; LG = lingual gyrus; PreCG = precentral gyrus; $\mathrm{SMG}=$ supramarginal gyrus; $\mathrm{FG}=$ fusiform gyrus; $\mathrm{p}<0.05$ corrected at cluster level; $\mathrm{LH}=$ left hemisphere, $\mathrm{RH}=$ right hemisphere. 
$[V H>H]$. In the visual-haptic dot pattern condition increased activation was found bilaterally in pCS, lateral pOCC and LG compared to the haptic condition.

$[V H>V]$. The bimodal as compared to the visual dot pattern condition showed increased activation in left-hemispheric frontal, somatosensory and visual regions; namely the medial frontal gyrus (MeFG), the pre- and postcentral gyrus (PrCG, PoCG), the supramarginal gyrus (SMG), pCS, lateral pOCC and the fusiform gyrus (FG) but also bilaterally in the basal ganglia, the thalamus and the cerebellum. The increased activation of early visual regions in the visual-haptic condition as contrasted to the visual condition is especially interesting considering that the visual information accessible to the subjects was the same in both the unimodal visual and the bimodal dot pattern condition.

$[V] \cap[H] \cap[V H>V] \cap[V H>H]$. A similar left lateralized region in pCS and lateral pOCC was identified in the conjunction contrast asking for brain areas that respond to visual and haptic dot pattern exploration but significantly more so to congruent visual-haptic texture information (see Figure 7).

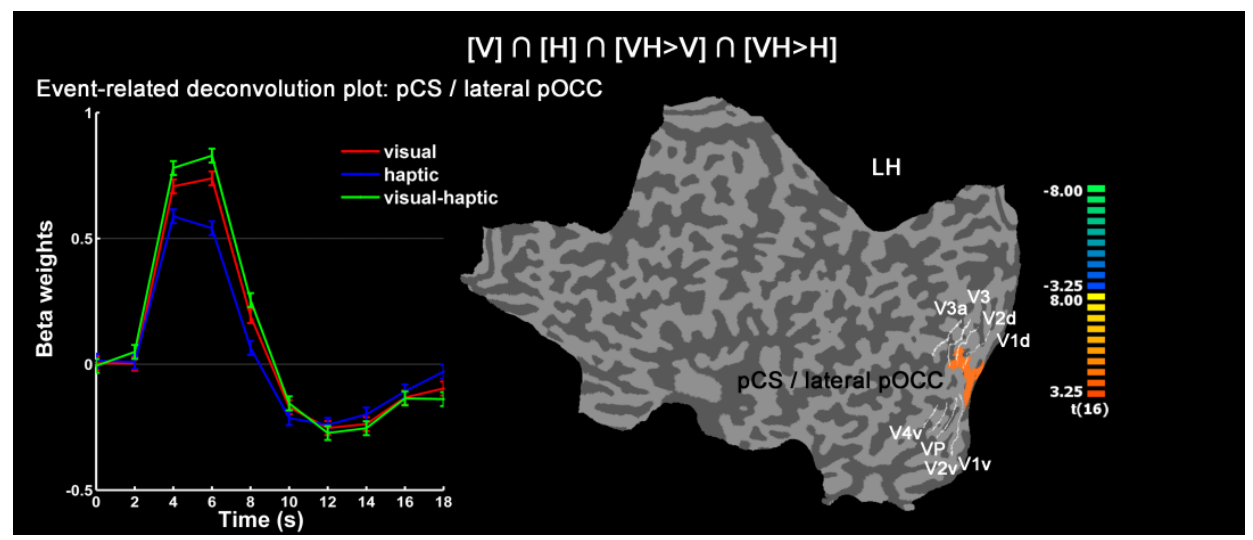

Figure 7. Increased activation for bimodal as compared to both unimodal dot pattern conditions. Right: Random effect map of the conjunction contrast projected on a flattened surface reconstruction of a single representative subject for which early visual areas were delineated by retinotopic mapping; $\mathrm{V}=$ dot pattern information in visual modality (haptic control stimulus); $\mathrm{H}=$ dot pattern information in haptic modality (visual control stimulus); $\mathrm{VH}=$ matching dot pattern information in visual and haptic modality; $\mathrm{pCS}=$ posterior calcarine sulcus; $\mathrm{pOCC}=$ posterior occipital cortex; $\mathrm{p}<0.05$ corrected at cluster level; $\mathrm{LH}=$ left hemisphere. Left: Event-related deconvolution plot depicting an increased BOLD response for the visual-haptic dot pattern condition compared to both unimodal dot pattern conditions in the occipital cluster identified in the conjunction contrast; bars represent the standard error of the mean (SEM); 0 on the time axis represents stimulus onset. 
Differences related to parametric dot pattern variation within each modality condition

Model 2 Test for modulation of the HRF by objective texture characteristics (mean inter-dot spacing).

All parametric effects for this model are summarized in Figure 8 and Table 3.

Table 3. Differences related to parametric dot pattern variation within each modality condition (models 2-4).

\begin{tabular}{|c|c|c|c|c|c|c|c|}
\hline $\begin{array}{l}\text { Para- } \\
\text { metric } \\
\text { modu- } \\
\text { lation }\end{array}$ & Contrast & Region & $\begin{array}{l}\text { Hemi- } \\
\text { sphere }\end{array}$ & $\mathrm{x}$ & $\mathrm{y}$ & $\mathrm{Z}$ & $\mathrm{t}$ \\
\hline \multicolumn{8}{|c|}{$\begin{array}{l}\text { Inter-dot spacing of textures } \\
{[\text { Hmain }] \cap\left[\mathrm{Hpara}^{+}\right]}\end{array}$} \\
\hline & & pOCC & $\mathrm{R}$ & 8 & -95 & 0 & 4.74 \\
\hline & & Cerebellum & $\mathrm{R}$ & 2 & -44 & -6 & 6.09 \\
\hline & [VHmai & $\cap[$ VHpara +$]$ & & & & & \\
\hline & & $\begin{array}{l}\text { pCS, lateral pOCC, } \\
\text { LG }\end{array}$ & $\mathrm{L} / \mathrm{R}$ & -13 & -95 & -6 & 6.99 \\
\hline & & aLG & $\mathrm{R}$ & 11 & -56 & 3 & 5.33 \\
\hline & & PreCG, PoCG, IPL & $\mathrm{L}$ & -46 & -32 & 60 & 5.96 \\
\hline
\end{tabular}

Roughness perceived by touch

[VHmain] $\cap[$ VHpara+ $]$

$\begin{array}{llrrrr}\text { pCS } & \text { L } & -13 & -89 & -15 & 4.33 \\ \text { PoCG } & \text { L } & -52 & -29 & 54 & 4.91\end{array}$

Spatial density perceived by vision

[VHmain] $\cap$ [VHpara-]

\begin{tabular}{llrrrr} 
Lateral pOCC & $\mathrm{R}$ & 17 & -83 & -12 & 4.62 \\
pCS, lateral pOCC, & $\mathrm{L}$ & -13 & -92 & -6 & 7.41 \\
LG & & & & & \\
aLG & $\mathrm{R}$ & 8 & -56 & 3 & 4.92 \\
PoCG, IPL & $\mathrm{L}$ & -49 & -29 & 54 & 5.43 \\
\hline
\end{tabular}

Note. $\mathrm{H}=$ dot pattern information in haptic modality (visual control stimulus); $\mathrm{VH}=$ matching dot pattern information in visual and haptic modality; $\mathrm{pOCC}=$ posterior occipital gyrus; $\mathrm{pCS}=$ posterior calcarine sulcus; $\mathrm{aLG}=$ anterior lingual gyrus; $\mathrm{PreCG}=$ precentral gyrus; $\mathrm{PoCG}=$ postcentral gyrus; $\mathrm{IPL}=$ inferior parietal lobule; $\mathrm{x}, \mathrm{y}, \mathrm{z}=$ Talairach coordinates of peak voxel; $\mathrm{t}=$ peak $\mathrm{t}$ value; $\mathrm{R}=$ right hemisphere; $\mathrm{L}=$ left hemisphere; $\mathrm{p}<0.05$ corrected at cluster level. 


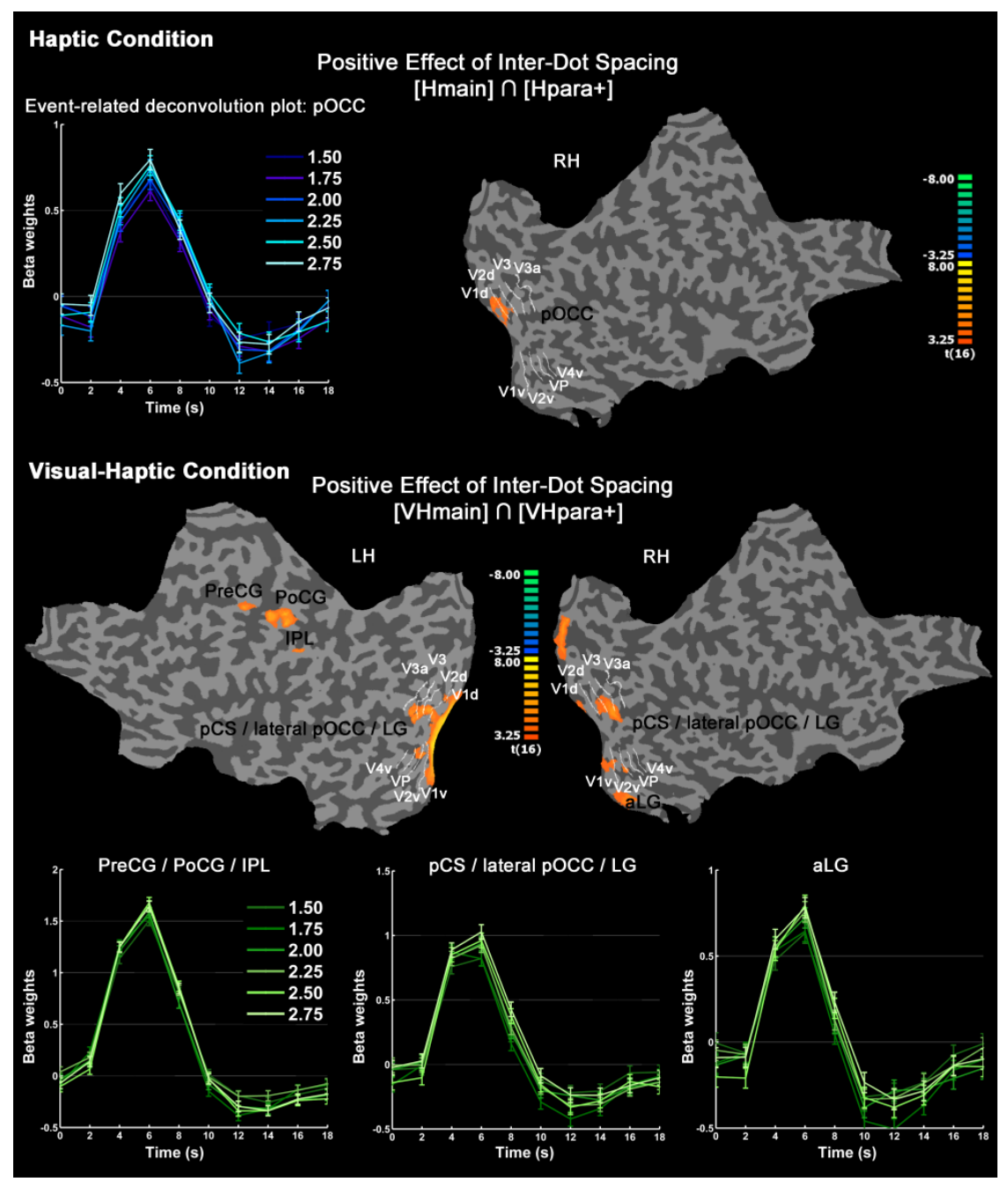

Figure 8. Brain regions showing graded BOLD responses with varying objective texture characteristics (inter-dot spacing) in different modality conditions. Random effects maps of the conjunction contrast of the main modality predictor and the positive parametric predictor projected on a flattened surface reconstruction of a single representative subject for which early visual areas were delineated by retinotopic mapping; $\mathrm{H}=$ dot pattern information in haptic modality (visual control stimulus); $\mathrm{VH}=$ matching dot pattern information in visual and haptic modality; pOCC $=$ posterior occipital cortex; PreCG = precentral gyrus; PoCG = postcentral gyrus; IPL = inferior parietal lobule; $\mathrm{pCS}=$ posterior calcarine sulcus; $\mathrm{aLG}=$ anterior lingual gyrus; $\mathrm{p}<0.05$ corrected at cluster level; $\mathrm{LH}=$ left hemisphere, $\mathrm{RH}=$ right hemisphere. Event-related deconvolution plots depicting a small linear effect of the inter-dot spacing of the textures on the BOLD response in brain regions identified by the conjunction contrast of the main and the parametric predictor; bars represent the standard error of the mean (SEM). 
[Vmain] $\cap$ [Vpara]. No parametric effects were found in the visual dot pattern condition.

[Hmain] $\cap$ [Hpara]. However, a small but significant positive parametric effect of inter-dot spacing was identified in the haptic condition in the right-hemisphere in pOCC as well as in the right cerebellum.

[VHmain] $\cap$ [VHpara]. For the congruent visual-haptic condition more extended positive parametric effects of inter-dot spacing were identified, namely bilaterally in pCS, the lateral pOCC and LG, right hemispheric in anterior LG (aLG) and in a contralateral cluster comprising PreCG and PoCG and the inferior parietal lobule (IPL). These parametric effects were quite small, as shown in the deconvolution plots of Figure 8, but the slight linear increase of the BOLD response with increasing inter-dot spacing is nevertheless visible.

Models 3 and 4 Test for modulation of the HRF by individually perceived texture characteristics (haptic roughness and visual spatial density).

All parametric effects for these models are summarized in Table 3 and Figure 9.

[Vmain] $\cap$ [Vpara], [Hmain] $\cap$ [Hpara]. Interestingly, no modulation by perceived roughness and spatial density was found in either unimodal dot pattern condition.

[VHmain] $\cap$ [VHpara]. However, there were parametric effects of these subjective measures in the visual-haptic condition. They resemble the effects found for the physical inter-dot spacing in this condition albeit with a more limited spatial extent. Left lateralized positive effects of perceived roughness on the BOLD signal were found in pCS and PoCG. Negative parametric effects of spatial density were shown in the left pCS and LG, the bilateral pOCC, in the right aLG as well as in the left PoCG and IPL. 


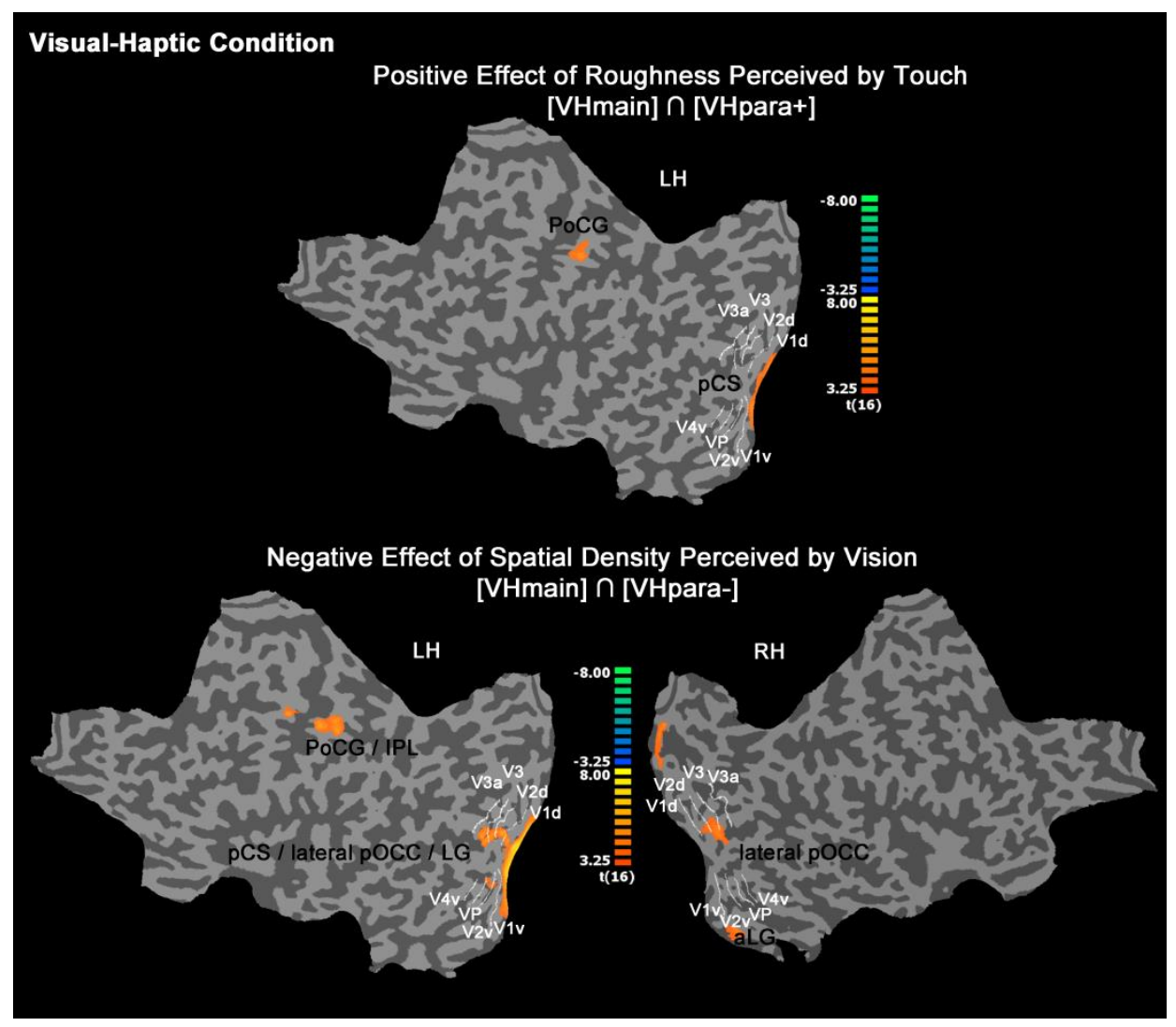

Figure 9. Brain regions showing graded BOLD responses with varying subjective texture characteristics (roughness perceived by touch, spatial density perceived by vision) in the bimodal dot pattern condition. Random effects maps of the conjunction contrast of the main modality predictor and the positive or negative parametric predictor projected on a flattened surface reconstruction of a single representative subject for which early visual areas were delineated by retinotopic mapping; $\mathrm{VH}=$ matching dot pattern information in visual and haptic modality; PoCG = postcentral gyrus; pCS = posterior calcarine sulcus; IPL = inferior parietal lobule; $\mathrm{pOCC}=$ posterior occipital cortex; aLG = anterior lingual gyrus; $\mathrm{p}<0.05$ corrected at cluster level; $\mathrm{LH}=\mathrm{left}$ hemisphere, $\mathrm{RH}=$ right hemisphere.

\section{DISCUSSSION}

\section{Differences between unimodal and bimodal dot pattern conditions}

Texture perception generated a very similar activation pattern irrespective of the modality in which texture information was varied, with activation overlapping primarily in early and higher-order visual, somatosensory and motor regions. These activations coincided with brain 
regions that were previously suggested to play a role in haptic and visual texture perception, namely the parietal operculum and insula, regions in the early visual cortex as well as the middle occipital gyrus, the collateral sulcus and the posterior fusiform and lingual gyrus (Cant and Goodale, 2011; Cant and $\mathrm{Xu}$, 2012; Cavina-Pratesi et al., 2010a; Deshpande et al., 2008; Kitada et al., 2005; Sathian et al., 2011; Stilla and Sathian, 2008). The consistent spatial overlap across conditions is in line with findings from Mouraux et al. (2011) who reported that a wide network of brain regions shows multimodal activations, i.e. responses that can be elicited by stimuli from more than a single sensory modality. Furthermore, since we only varied the sensory information content between the different conditions but not the stimulated modalities, the even greater spatial consistency, including also early sensory regions in the current study, is not surprising. In contrast to other studies we did not find a strong engagement of prefrontal areas (Kitada et al., 2005; Simões-Franklin et al., 2011). One potential explanation for this difference is the engagement in an active cognitive task in most paradigms versus the mere exploration of the textures in the experiment presented here.

Despite the close resemblance of the activation maps in the different texture variation conditions, we did find a change of the BOLD response in both visual and somatosensory cortex when subjects explored dot patterns only visually, only haptically or bimodally. Irrespective of the visual input, haptic dot pattern exploration induced increased activation in the contralateral PoCG, the putative location of the primary somatosensory cortex. This result indicates independence of the average dot-pattern-elicited activation level in PoCG from other sensory input. It is likely that perception of dot patterns is mediated mainly by slowly adapting type 1 (SA1) mechanoreceptive afferents (Bensmaia et al., 2006; Bergmann Tiest, 2010; Hollins et al., 2006). The different SA1 input during dot pattern versus smooth texture exploration might be represented in a different cortical activation pattern in the postcentral cortex (Chen et al., 2001; Friedman et al., 2004). Presumably, these differences would be at a finegrained level (Pei et al., 2009) unlikely to be discovered by the data acquisition parameters used in our study, i.e. a voxel size of $3.5 \times 3.5 \times 3.5 \mathrm{~mm}^{3}$, and the smoothing applied to the data. Alternatively, the literature demonstrates that attention modulation of sensory processing exists already as early as in the primary somatosensory cortex (e.g. Dionne et al., 2010; Karns and Knight, 2009; Sterr et al., 2007; for a review see Johansen-Berg and Lloyd, 2000). It is possible that the more salient dot pattern textures resulted in a shift of attention towards haptic texture exploration as compared to the visual condition in which only a smooth texture was explored by touch. In summary the comparison of all modality conditions indicates that visual information has no effect on haptic texture processing in somatosensory regions.

In early visual cortex the picture is slightly different. Here we also find an increase of the BOLD signal with texture variation in the visual condition but the size of the increase depends on the type of haptic information provided, 
reaching its maximum when matching haptic texture information is available. The increased activation in the visual dot pattern trials as compared to the presentation of the visual smooth control stimulus is expected, since dot pattern textures have more features and hence a higher contrast than the smooth control stimulus. This dependence of the BOLD signal on contrast changes of visual stimuli in early visual regions was already presented by Boynton et al. (1996) and later confirmed by others (e.g. Buracas et al., 2005).

However, the increased BOLD response in the bimodal dot pattern condition can only be explained by the matching haptic texture information, since the visual input was the same in the visual and in the visual-haptic condition. This indicates a crossmodal interaction of haptic and visual information in early visual cortex, and confirms our hypothesis that haptic information can modulate visual information processing already at a very early processing stage (as suggested by Lunghi et al., 2010). The existence of crossmodal effects in the visual cortex was reported by several studies in the last decade, focusing for example on target detection (Bauer et al., 2009), spatial attention (Macaluso et al., 2000), shape perception (Amedi et al., 2001; Lacey et al., 2009), tactile distance judgments (Merabet et al., 2004, 2007), tactile threshold-tracking (Nordmark et al., 2012) and tactile orientation discrimination (Sathian et al., 1997; Zangaladze et al., 1999). However, the present study is to our knowledge the first one showing a direct influence of haptic information on visual processing in early visual cortex for a microspatial task, i.e. by dealing with small-scale surface deviations as in comparison to large-scale features like shape and size (macrospatial) (Roland and Mortensen, 1987).

This result is consistent with studies emphasizing that early visual regions play a role in both visual and haptic texture perception, speculatively by housing bisensory neurons or intermingled populations of modality-specific neurons (Kim et al., 2012; Sathian et al., 2011; Stilla and Sathian, 2008). Several studies used measures like superadditivity or inverse effectiveness to address the question of neuronal or areal convergence in brain regions activated by stimuli from multiple sensory modalities (e.g. Calvert et al., 2000; Kim et al., 2012). For a recent discussion of different measures used to investigate multisensory phenomena in fMRI studies see James and Stevenson (2012). Our study was not designed to answer this question for visual-haptic texture processing. No purely unimodal stimuli were presented which would be needed to compare the BOLD response of these stimuli to responses elicited by bimodal stimuli. However, it can be concluded from our results that haptic texture information influences the processing of visual information in early occipital regions. Further implications of this finding with respect to neuronal or areal convergence have to be investigated using experimental designs optimized for direct comparisons of unimodal and bimodal texture processing. 


\section{Effects of parametric dot pattern variation within each modality condition}

The analysis of the behavioral data showed a linear increase of roughness perceived by touch for textures with increasing average dot-spacing and a linear decrease of visual spatial density. The results indicate that on average subjects were able to discriminate the roughness as well as the density of all textures. These results were expected from earlier work of our (Eck et al., 2011) and other groups (Merabet et al., 2004, 2007). However, the individual ratings, displayed in Figure 4, show that there are nevertheless small interindividual differences in haptic roughness as well as visual density perception. Therefore, we decided to use both subjective texture characteristics and the objective inter-dot spacing of the textures for the parametric modulation of the HRF in all modality conditions. The parametric modulation of the BOLD signal by all three measures resulted in very similar spatial characteristics of the statistical maps, as expected by the high correlation between the ratings and the objective texture characteristics. Nevertheless, the variation of the BOLD response was captured slightly better by inter-dot spacing than by perceived texture characteristics, as indicated by the increased spatial extent of the former mentioned parametric effects. Therefore, we will focus on discussing the effects of inter-dot spacing. In general, the parametric effects of texture variation on the BOLD response in this study were rather small. However, these effects are nevertheless remarkable for the following reasons. First, there were only very small differences between all dot pattern textures and although subjects were able to differentiate the textures after the fMRI experiment, the differences were not large enough to expect tremendous differences in the evoked BOLD response. This is especially true for the fast event-related stimulus presentation that was chosen to prevent subjects from actively judging the texture dimensions and to avoid boredom. Second, subjects were only instructed to focus on the simultaneous visual and haptic texture exploration. While it cannot be excluded that some evaluation processes occurred, active cognitive processing is expected to be absent or highly limited. Very similar instructions resulted in a lack of parametric responses in the cortex in previous unimodal studies (Kitada et al., 2005). Despite the rather subtle texture differences, we saw small activation changes with varying texture characteristics in both early visual and somatosensory cortex. A parametric variation of the BOLD response in the left PoCG was exclusively found for matching visual and haptic texture information (bimodal dot pattern condition). This indicates that visual information has an effect on relative activation differences elicited by varying tactile texture characteristics in early somatosensory cortex. Crossmodal influences on somatosensory cortex were already reported by Meehan and Staines (2009) and Dionne et al. (2010), using vibrotactile stimulation. Both studies found variations of somatosensory elicited BOLD responses in PoCG with visual information. 
Interestingly, we failed to find a parametric effect on the BOLD signal of the visual cortex in the visual texture variation condition. Only with varying haptic texture information a parametric effect was observed in the right posterior OCC, independent of the visual information provided. One explanation for the parametric effect in the visual cortex in a haptic texture variation condition would be visual imagery. However, if this interpretation applies we would have expected a similar parametric effect in the visual condition, which was not the case in the present study. An alternative explanation is that the visual cortex responds not only to visual but also to a certain extent to tactile stimulation. This result contradicts the traditional view that early sensory cortices are limited to information processing from a single modality. However, as already indicated above, many studies have demonstrated the same pattern of results, i.e. responsiveness of the visual cortex to non-visual stimuli (for a summary see Sathian and Lacey (2007) and Lacey et al. (2008)). These studies focused mainly on shape perception (Amedi et al., 2001; Kassuba et al., 2013; Kim and James, 2009), describing regions in the occipital cortex, i.e. the lateral occipital complex (LOC), that are not only responsive to visual object perception but also to haptic perception of 3D objects. Importantly, these findings were not a mere result of visual imagery as expected at the beginning of this line of research (Deshpande et al., 2010; Lacey et al., 2010). In the last years even more studies were published suggesting a contribution of the visual cortex also to other tactile tasks, as for example grating orientation discrimination (Zhang et al., 2005), dot pattern judgments (Merabet et al., 2004, 2007) and texture matching (Stilla and Sathian, 2008). However, we did not only find a parametric effect of inter-dot spacing in the visual cortex, when only haptic dot patterns were presented; additionally, we observed that the parametric effect on the BOLD signal expanded to the left pOCC as well as to the pCS and the lingual gyrus when haptic and visual texture characteristics were varied congruently. This clearly indicates an interaction of visual and haptic dot pattern information in early visual cortex.

We did not find any parametric effects in the classical brain regions implicated in texture perception, as for example the collateral sulcus and the parietal operculum and the insula; while Kitada et al. (2005) reported parametric effects of tactile roughness estimation in the latter two areas as well as in prefrontal regions. However, in contrast to previous studies we asked subjects to explore the textures without any active cognitive task. We would expect an involvement of these higher-order brain regions with increasing task demands, e.g. when an evaluation of the presented textures along a specific dimension or a comparison of the textures is necessary, consistent with the report of Kitada. The parametric effects on the BOLD response in early sensory cortices in our study rather indicate a modulation already at an early processing stage without cognitive evaluation demands. This view can be well integrated in the interpretation of Hiramatsu et al. (2011), that early visual regions, i.e. V1/V2, V3 contain image-based representations of material properties rather than 
higher-region perceptual representations. Retinotopic information was only available for two subjects in our sample and the retinotopic map of one of these subjects was used to present the results. Although we cannot be certain in which early visual regions the significant activations were located, the exemplary retinotopic map suggests an involvement of V1-V3. This is partly in accordance with the findings of Merabet et al. (2007) who showed a clear activation of primary visual cortex (V1) by tactile stimulation and deactivation of extrastriate regions (V2, V3, V3a, hV4). Individuals were blindfolded in Merabet's experiment, while subjects in the current study received nonmatching and matching visual stimulation. This additional visual stimulation would be a plausible explanation for the difference in the findings across both studies. The connectivity analyses by Sathian et al. (2011), showing that tactile texture information is first processed in the PoCG before flowing to higherorder cortices, also indicate that the parametric effects of varying texture characteristics in the present study represent a rather early and automatic cortical processing stage. The result that inter-dot spacings rather than perceived texture differences were slightly better qualified to capture parametric BOLD modulation with texture variation also points to that interpretation.

Taken together, the results of the parametric analyses confirm our hypothesis that the additional presentation of matching visual texture information is sufficient to observe parametric effects of varying texture characteristics on the BOLD response, even when subjects are not required to evaluate the presented textures. This extends the findings of Kitada et al. (2005), who did not find a parametric variation of the BOLD signal when subjects explored the textures tactually without a judgment task. Crossmodal interaction effects of visual and haptic texture information in early sensory cortices are the probable cause of the differences between both studies.

\section{Integration of results}

To summarize the results above, we found an effect of matching visualhaptic texture information in early visual and somatosensory regions. However, the kind of crossmodal interaction was slightly different in both sensory cortices. In the contralateral somatosensory cortex (PoCG) the activation level did not change between the presentation of unimodal haptic and bimodal visual-haptic dot pattern information. However, in the bimodal dot pattern condition, small changes in the BOLD response were detected with varying texture characteristics, indicating a crossmodal effect on relative differences in the BOLD signal of the contralateral somatosensory cortex in response to different textures.

The average BOLD response in a posterior occipital cluster in the left hemisphere increased when bimodal as compared to unimodal visual or haptic dot pattern information was provided, indicating a crossmodal effect on the 
overall signal in the left early visual cortex. Parametric effects on the BOLD response in early visual cortex were already found when only haptic texture information was varied, though right-lateralized only. When matching visual dot pattern information was added, the parametric effects were more pronounced in the same hemisphere and extended also to the left visual cortex. This indicates, as with the somatosensory cortex, a crossmodal effect on relative differences in the BOLD signal of the visual cortex in response to different textures.

In contrast to the classical view that different sensory information converges only at higher association areas, our findings lead us to believe that crossmodal interactions occur already at an early automatic stage of texture processing. The reasons for that interpretation are twofold. First, overall and relative effects of matching visual-haptic texture variation were primarily observed in early sensory cortices without a contribution of prefrontal regions and higher sensory cortices. Second, inter-dot spacing was slightly better qualified to capture parametric BOLD modulation with dot pattern variation than perceived texture characteristics.

Our study design does not allow us to identify the source of the crossmodal interactions; however we propose two possible explanations. The first would be hierarchical feedback of top-down influences from higher sensory areas on early sensory cortices. This explanation is consistent with the finding of projections from parietal association areas to V1 and V2 in the macaque (Borra and Rockland, 2011) and with connectivity analyses by Sathian et al. (2011) showing that visual texture information flows from the higher-order fusiform gyrus to early visual cortex. However, no visual texture information flow to early somatosensory regions was reported by the latter study. Hence, the fact that only matching visual-haptic texture information induced a parametric modulation of the BOLD response in the contralateral somatosensory cortex (PoCG) cannot be explained in this way. An alternative, and in our opinion more likely interpretation would be direct or indirect cortico-cortical connections between early sensory cortices (Cappe and Barone, 2005), explaining the crossmodal texture effects in both early somatosensory and visual cortex. This is partly in accordance with the results by Deshpande et al. (2008) and Sathian et al. (2011) who showed a direct flow of haptic texture information from the PoCG to bilateral early visual cortex.

\section{CONCLUSION}

The present study aimed to investigate whether cortical processing in early sensory cortices changes when matching as compared to non-matching visual-haptic texture information is provided. Earlier research has shown that crossmodal interactions for texture perception exist at the behavioral level. However, neuroimaging studies focused primarily on visual and haptic texture 
processing separately, allowing to investigate only the overlap of both sensory processing streams. The variation of texture information in a bimodal task in the present study permitted the exploration of crossmodal interactions at the cortical level. More specifically, we hypothesized that parametric effects on the BOLD signal can be expected with varying texture characteristics when matching visual-haptic textures are explored, even when a cognitive evaluation of the textures is not required.

Indeed, both early visual and somatosensory cortex showed crossmodal interactions of visual-haptic texture processing. These effects were slightly different in both sensory cortices, with bimodal visual-haptic dot pattern information increasing the average activation level as well as inducing relative differences of the BOLD signal with varying texture characteristics in early visual cortex, and only the latter effect in somatosensory cortex.

The results presented here challenge the notion that the cortical processing of texture information in the visual and haptic modality is independent of each other. Rather than a rigid sensory separation between classical visual and somatosensory brain regions, the results suggest a flexible cooperation of sensory cortices.

\section{ACKNOWLEDGMENTS}

The research leading to these results has received funding from the European Community's Seventh Framework Programme FP7/2007-2013 under grant agreement number PITN-GA-2008-214728. We also gratefully acknowledge the support of the BrainGain Smart Mix Programme of the Netherlands Ministry of Economic Affairs and the Netherlands Ministry of Education, Culture and Science. We would like to thank Ron Hellenbrand and Joost Mulders for their help with the stimulus creation. 


\section{REFERENCES}

Amedi, A., Malach, R., Hendler, T., Peled, S., Zohary, E., 2001. Visuo-haptic objectrelated activation in the ventral visual pathway. Nat. Neurosci. 4, 324-330.

Bauer, M., Oostenveld, R., Fries, P., 2009. Tactile stimulation accelerates behavioral responses to visual stimuli through enhancement of occipital gamma-band activity. Vision Res. 49, 931-942.

Bensmaia, S.J., Craig, J.C., Yoshioka, T., Johnson, K.O., 2006. SA1 and RA afferent responses to static and vibrating gratings. J. Neurophysiol. 95, 1771-1782.

Bergmann Tiest, W.M., 2010. Tactual perception of material properties. Vision Res. 50, 2775-2782.

Borra, E., Rockland, K.S., 2011. Projections to early visual areas V1 and V2 in the calcarine fissure from parietal association areas in the macaque. Front. Neuroanat. 5, 1-12.

Boynton, G.M., Engel, S.A., Glover, G.H., Heeger, D.J., 1996. Linear systems analysis of functional magnetic resonance imaging in human V1. J. Neurosci. 16, 42074221.

Buracas, G.T., Fine, I., Boynton, G.M., 2005. The relationship between task performance and functional magnetic resonance imaging response. J. Neurosci. 25, 3023-3031.

Burton, H., Sinclair, R.J., Wingert, J.R., Dierker, D.L., 2008. Multiple parietal operculum subdivisions in humans: tactile activation maps. Somatosens. Mot. Res. 25, 149-162.

Calvert, G.A., Campbell, R., Brammer, M.J., 2000. Evidence from functional magnetic resonance imaging of crossmodal binding in the human heteromodal cortex. Curr. Biol. 10, 649-657.

Cant, J.S., Goodale, M.A., 2011. Scratching beneath the surface: new insights into the functional properties of the lateral occipital area and parahippocampal place area. J. Neurosci. 31, 8248-8258.

Cant, J.S., Goodale, M.A., 2007. Attention to form or surface properties modulates different regions of human occipitotemporal cortex. Cereb. Cortex 17, 713731.

Cant, J.S., Xu, Y., 2012. Object ensemble processing in human anterior-medial ventral visual cortex. J. Neurosci. 32, 7685-7700.

Cappe, C., Barone, P., 2005. Heteromodal connections supporting multisensory integration at low levels of cortical processing in the monkey. Eur. J. Neurosci. 22, 2886-2902.

Cavina-Pratesi, C., Kentridge, R.W., Heywood, C.A., Milner, A.D., 2010a. Separate processing of texture and form in the ventral stream: evidence from fMRI and visual agnosia. Cereb. Cortex 20, 433-446.

Cavina-Pratesi, C., Kentridge, R.W., Heywood, C.A., Milner, A.D., 2010b. Separate channels for processing form, texture, and color: evidence from fMRI adaptation and visual object agnosia. Cereb. Cortex 20, 2319-2332.

Chen, L.M., Friedman, R.M., Ramsden, B.M., LaMotte, R.H., Roe, A.W., 2001. Finescale organization of SI (area $3 b$ ) in the squirrel monkey revealed with intrinsic optical imaging. J. Neurophysiol. 86, 3011-3029.

Connor, C.E., Hsiao, S.S., Phillips, J.R., Johnson, K.O., 1990. Tactile roughness: neural codes that account for psychophysical magnitude estimates. J. Neurosci. 10, 3823-3836. 
Dépeault, A., Meftah, E.-M., Chapman, C.E., 2009. Tactile perception of roughness: raised-dot spacing, density and disposition. Exp. Brain Res. 197, 235-244.

Deshpande, G., Hu, X., Lacey, S., Stilla, R., Sathian, K., 2010. Object familiarity modulates effective connectivity during haptic shape perception. Neuroimage 49, 1991-2000.

Deshpande, G., Hu, X., Stilla, R., Sathian, K., 2008. Effective connectivity during haptic perception: A study using Granger causality analysis of functional magnetic resonance imaging data. Neuroimage 40, 1807-1814.

Dionne, J.K., Meehan, S.K., Legon, W., Staines, W.R., 2010. Crossmodal influences in somatosensory cortex: interaction of vision and touch. Hum. Brain Mapp. 31, $14-25$.

Eck, J., Kaas, A.L., Goebel, R., 2011. The effect of sensory modality and previous experience on perceived roughness. Paper presented at the World Haptics Conference (WHC). IEEE, Instanbul, Turkey.

Fikes, T.G., Klatzky, R.L., Lederman, S.J., 1994. Effects of object texture on precontact movement time in human prehension. J. Mot. Behav. 26, 325-332.

Forman, S.D., Cohen, J.D., Fitzgerald, M., Eddy, W.F., Mintun, M.A., Noll, D.C., 1995. Improved assessment of significant activation in functional magnetic resonance imaging (fMRI): use of a cluster-size threshold. Magn. Reson. Med. 33, 636647.

Friedman, R.M., Chen, L.M., Roe, A.W., 2004. Modality maps within primate somatosensory cortex. Proc. Natl. Acad. Sci. U. S. A. 101, 12724-12729.

Goebel, R., Esposito, F., Formisano, E., 2006. Analysis of functional image analysis contest (FIAC) data with Brainvoyager QX: from single-subject to cortically aligned group general linear model analysis and self-organizing group independent component analysis. Hum. Brain Mapp. 27, 392-401.

Guzman-Martinez, E., Ortega, L., Grabowecky, M., Mossbridge, J., Suzuki, S., 2012. Interactive coding of visual spatial frequency and auditory amplitudemodulation rate. Curr. Biol. 22, 383-388.

Hiramatsu, C., Goda, N., Komatsu, H., 2011. Transformation from image-based to perceptual representation of materials along the human ventral visual pathway. Neuroimage 57, 482-494.

Hollins, M., Lorenz, F., Harper, D., 2006. Somatosensory coding of roughness: the effect of texture adaptation in direct and indirect touch. J. Neurosci. 26, 55825588 .

James, T.W., Stevenson, R.A., 2012. The use of fMRI to assess multisensory integration. In: Murray, M.M., Wallace, M.T. (Eds.), The Neural Bases of Multisensory Processes. CRC Press, Boca Raton (FL).

Johansen-Berg, H., Lloyd, D.M., 2000. The physiology and psychology of selective attention to touch. Front. Biosci. 5, 894-904.

Kaas, A.L., van Mier, H., Visser, M., Goebel, R., 2013. The neural substrate for working memory of tactile surface texture. Hum. Brain Mapp. 34, 1148-1162.

Kao, M.-H., Mandal, A., Lazar, N., Stufken, J., 2009. Multi-objective optimal experimental designs for event-related fMRI studies. Neuroimage 44, 849-856.

Karns, C.M., Knight, R.T., 2009. Intermodal auditory, visual, and tactile attention modulates early stages of neural processing. J. Cogn. Neurosci. 21, 669-683.

Kassuba, T., Klinge, C., Hölig, C., Röder, B., Siebner, H.R., 2013. Vision holds a greater share in visuo-haptic object recognition than touch. Neuroimage 65, $59-68$. 
Kim, S., James, T.W., 2009. Enhanced effectiveness in visuo-haptic object-selective brain regions with increasing stimulus salience. Hum. Brain Mapp. 31, 678693

Kim, S., Stevenson, R.A., James, T.W., 2012. Visuo-haptic neuronal convergence demonstrated with an inversely effective pattern of BOLD activation. J. Cogn. Neurosci. 24, 830-842.

Kitada, R., Hashimoto, T., Kochiyama, T., Kito, T., Okada, T., Matsumura, M., Lederman, S.J., Sadato, N., 2005. Tactile estimation of the roughness of gratings yields a graded response in the human brain: an fMRI study. Neuroimage 25, 90-100.

Kitada, R., Kito, T., Saito, D.N., Kochiyama, T., Matsumura, M., Sadato, N., Lederman, S.J., 2006. Multisensory activation of the intraparietal area when classifying grating orientation: a functional magnetic resonance imaging study. J. Neurosci. 26, 7491-7501.

Lacey, S., Flueckiger, P., Stilla, R., Lava, M., Sathian, K., 2010. Object familiarity modulates the relationship between visual object imagery and haptic shape perception. Neuroimage 49, 1977-1990.

Lacey, S., Sathian, K., 2008. Haptically evoked activation of visual cortex. In: Grunwald, M. (Ed.), Human Haptic Perception: Basics and Applications. Birkhäuser Basel, Basel, 251-257.

Lacey, S., Tal, N., Amedi, A., Sathian, K., 2009. A putative model of multisensory object representation. Brain Topogr. 21, 269-274.

Lederman, S.J., Abbott, S.G., 1981. Texture perception: studies of intersensory organization using a discrepancy paradigm and visual versus tactual psychophysics. J. Exp. Psychol. Hum. Percept. Perform. 7, 902-915.

Lunghi, C., Binda, P., Morrone, M.C., 2010. Touch disambiguates rivalrous perception at early stages of visual analysis. Curr. Biol. 20, 143-144.

Macaluso, E., Frith, C.D., Driver, J., 2000. Modulation of human visual cortex by crossmodal spatial attention. Science 289, 1206-1208.

Meehan, S.K., Staines, W.R., 2009. Task-relevance and temporal synchrony between tactile and visual stimuli modulates cortical activity and motor performance during sensory-guided movement. Hum. Brain Mapp. 30, 484-496.

Merabet, L.B., Swisher, J.D., McMains, S.A., Halko, M.A., Amedi, A., Pascual-Leone, A., Somers, D.C., 2007. Combined activation and deactivation of visual cortex during tactile sensory processing. J. Neurophysiol. 97, 1633-1641.

Merabet, L., Thut, G., Murray, B., Andrews, J., Hsiao, S., Pascual-Leone, A., 2004. Feeling by sight or seeing by touch? Neuron 42, 173-179.

Mouraux, A., Diukova, A., Lee, M.C., Wise, R.G., Iannetti, G.D., 2011. A multisensory investigation of the functional significance of the "pain matrix". Neuroimage 54, 2237-2249.

Nordmark, P.F., Pruszynski, J.A., Johansson, R.S., 2012. BOLD responses to tactile stimuli in visual and auditory cortex depend on the frequency content of stimulation. J. Cogn. Neurosci. 24, 2120-2134.

Pei, Y.-C., Denchev, P.V., Hsiao, S.S., Craig, J.C., Bensmaia, S.J., 2009. Convergence of submodality-specific input onto neurons in primary somatosensory cortex. J. Neurophysiol. 102, 1843-1853.

Peuskens, H., Claeys, K.G., Todd, J.T., Norman, J.F., van Hecke, P., Orban, G.A., 2004. Attention to 3-D shape, 3-D motion, and texture in 3-D structure from motion displays. J. Cogn. Neurosci. 16, 665-682. 
Picard, D., 2006. Partial perceptual equivalence between vision and touch for texture information. Acta Psychol. (Amst) 121, 227-248.

Roland, P.E., Mortensen, E., 1987. Somatosensory detection of microgeometry, macrogeometry and kinesthesia in man. Brain Res. Rev. 12, 1-42.

Roland, P.E., O'Sullivan, B., Kawashima, R., 1998. Shape and roughness activate different somatosensory areas in the human brain. Proc. Natl. Acad. Sci. U.S.A. 95, 3295-3300.

Sathian, K., Lacey, S., 2007. Cross-modal involvement of visual cortex in tactile perception. In: Mast, F.W., Jäncke, L. (Eds.), Spatial Processing in Navigation, Imagery and Perception. Springer, New York, pp. 119-134.

Sathian, K., Lacey, S., Stilla, R., Gibson, G.O., Deshpande, G., Hu, X., LaConte, S., Glielmi, C., 2011. Dual pathways for haptic and visual perception of spatial and texture information. Neuroimage 57, 462-475.

Sathian, K., Zangaladze, A., Hoffman, J.M., Grafton, S.T., 1997. Feeling with the mind's eye. Neuroreport 8, 3877-3881.

Simões-Franklin, C., Whitaker, T.A., Newell, F.N., 2011. Active and passive touch differentially activate somatosensory cortex in texture perception. Hum. Brain Mapp. 32, 1067-1080.

Sterr, A., Shen, S., Zaman, A., Roberts, N., Szameitat, A., 2007. Activation of SI is modulated by attention: a random effects fMRI study using mechanical stimuli. Neuroreport 18, 607-611.

Stilla, R., Sathian, K., 2008. Selective visuo-haptic processing of shape and texture. Hum. Brain Mapp. 29, 1123-1138.

Zangaladze, A., Epstein, C.M., Grafton, S.T., Sathian, K., 1999. Involvement of visual cortex in tactile discrimination of orientation. Nature 401, 587-590.

Zhang, M., Mariola, E., Stilla, R., Stoesz, M., Mao, H., Hu, X., Sathian, K., 2005. Tactile discrimination of grating orientation: fMRI activation patterns. Hum. Brain Mapp. 25, 370-377. 



\section{Chapter 4}

\section{The effect of task instruction on haptic texture processing: The neural underpinning of roughness and spatial density perception}

Based on: Eck, J., Kaas, A.L., Mulders, J.L., Hausfeld, L., Kourtzi, Z., Goebel, R., 2013. The effect of task instruction on haptic texture processing: The neural underpinning of roughness and spatial density perception. Manuscript submitted for publication. 


\section{ABSTRACT}

Haptic perception of spatial density and roughness of dot pattern textures is characterized by different psychometric curves, i.e. a linear and a non-linear dependence on inter-element spacing respectively. Neuroimaging literature provides inconclusive evidence for a corresponding dissociation in the underlying cortical networks.

We used an integrative psychophysical and imaging approach to investigate this dissociation. Participants performed a haptic roughness and spatial density rating task on the same dot pattern textures. Multi-voxel pattern analysis was used to reveal activation changes depending on the task performed and to investigate which brain regions hold information indicative of the level of the perceived texture characteristic.

The results revealed an almost identical cortical network involved in both haptic rating tasks. However, more importantly, there was a dissociation in the brain regions contributing to the processing of the perceived level of both texture characteristics. While information about differences in perceived roughness was primarily available in higher-order cortices, i.e. the operculoinsular cortex and a ventral visual cortex region, information about perceived spatial density could be derived already from early somatosensory and visual regions. Furthermore, this study is to our knowledge the first to show a contribution of the ventral visual cortex to tactile roughness perception. 


\section{INTRODUCTION}

Textures can be described by several attributes such as rough, dense, soft, slippery, thick (Bergmann Tiest and Kappers, 2006; Gescheider et al., 2005; Hollins et al., 1993, 2000; Lederman et al., 1986; Picard et al., 2003). These subjective dimensions can be correlated or independent of each other. The haptic percept of dot patterns for example is well characterized by the perceived spatial density and by the perceived roughness of the texture (Merabet et al., 2004). The former represents a one to one mapping of the physical stimulus and is most likely related to the number of indentations per surface unit of the skin caused by the individual texture elements. The perceived roughness, on the other hand, is very likely a combination of two factors, i.e. the number of skin indentations per surface unit and the local skin displacement, which will be increasingly restricted by the skin's lack of compliance with decreasing inter-dot spacing (Eck et al., 2013b). Hence, perceived spatial density and perceived roughness are not independent of each other, both are influenced by the number of texture elements, but only the former one can be directly mapped to the physical stimulus space. A study by Merabet and colleagues (Merabet et al., 2004) showed that this distinction in the mapping of the two perceived texture dimensions to the stimulus space is also represented in the psychometric curves; while perceived spatial density linearly decreases with increasing inter-dot spacing of the dot patterns, perceived roughness follows an inverted $\mathrm{U}$-shape peaking at about $3 \mathrm{~mm}$ dot distance.

Neuroimaging literature suggests a similar distinction in the underlying cortical networks. However, most imaging studies did not systematically vary roughness or density levels, or focused only on a single perceptual dimension, leading to inconclusive findings. In one of the few comparative tactile roughness and spatial density estimation experiments, Merabet and colleagues (Merabet et al., 2007) reported higher activations in the intraparietal sulcus and in postcentral regions for density judgments. This indicates an involvement of early somatosensory and posterior parietal regions in tactile density estimation. Surprisingly, no regions showed the opposite effect, i.e. increased activations for the roughness estimation task. In contrast, a corresponding repetitive TMS (rTMS) study from the same group found clear task effects in both directions by showing that roughness perception was impaired by applying rTMS to the somatosensory cortex, while reports of spatial density were altered after rTMS to the occipital cortex (Merabet et al., 2004). Interestingly though, a similar disruptive effect for spacing discriminations was also reported for TMS targeting the somatosensory cortex in another study (Zangaladze et al., 1999). A difference in the employed stimulus set and the task instructions might have influenced the results (dot patterns vs. linear gratings and spacing estimation vs. discrimination). In an earlier fMRI study employing dot pattern textures we found clear parametric effects of stimulus spacing on the BOLD response in early occipital and somatosensory regions (Eck et al., 2013a), consistent with 
the reports of both Zangaladze and colleagues (Zangaladze et al., 1999) and Merabet et al. (Merabet et al., 2004). However, subjects in that study were instructed to merely explore the textures without any active judgment task, which could explain the finding of a predominant contribution of sensory cortices involved in a relatively early processing stage, rather than higher-order regions. Since the perceptual texture dimension spatial density can be considered a one to one mapping of the physical stimulus, it is not surprising that we found signal variations related to offline perceptual spatial density ratings in the same cortical regions that vary with physical density. However, spacing-related variations in the BOLD response were primarily observed in our previous study when congruent visual texture information was provided in addition to the haptic input, but not when haptic texture information was exclusively provided. This suggests that enhanced attention to the physical stimulus space evoked by a spatial density estimation task instruction might be sufficient to observe variations in cortical activation patterns with stimulus spacing in early sensory cortices also when only haptic information is available. This would be consistent with reports of a primarily early sensory cortex involvement in spacing estimation and discrimination of texture stimuli.

Studies comparing roughness discrimination to macrospatial tasks such as length and shape discrimination systematically reported an involvement of the parietal operculum (OP) in the former (Ledberg et al., 1995; O'Sullivan et al., 1994; Roland et al., 1998). These results were confirmed by experiments focusing on variations in surface roughness. Graded blood oxygen leveldependent (BOLD) effects were observed in the parietal operculum and the insula (Kitada et al., 2005; Simões-Franklin et al., 2011), but not in the primary somatosensory cortex. Kitada and colleagues also reported effects of stimulus roughness on activation in prefrontal regions, likely representing the cognitive processing of the task.

Although not completely consistent, overall the neuroimaging literature on haptic texture perception points to an early visual and somatosensory cortex contribution to density perception and an involvement of the operculo-insular cortex in roughness estimation. We suggest that this distinction in the cortical networks underlying density and roughness perception might be well explained by the above described differences in the mapping between the stimulus and perceptual space. To test this hypothesis, the current study used an integrative psychophysical and imaging approach, directly comparing a roughness estimation task to a spatial density estimation task of dot patterns varying in the mean center-to-center dot spacing, covering the full range of the psychometric curves. Our first aim was to characterize the cortical networks underlying density and roughness estimation. Second, we aimed to investigate in which of the previously implicated brain regions, cortical activation patterns vary with different levels of perceived texture characteristics, i.e. perceived roughness and density. Since multi-voxel pattern analysis (MVPA) is considered more sensitive than univariate data analysis methods to detect information in brain 
regions, we employed the former to reveal possible subtle differences in distributed activation patterns depending on the task context. Our third aim was to investigate to what extent similar effects can be observed for objective stimulus characteristics when subjects only focus on the stimulus exploration without a concurrent rating task.

As discussed above, whereas density estimation represents a one to one mapping between the physical and perceptual space, roughness estimation of dot pattern stimuli does not. It most likely requires integration of the number of skin indentations per surface unit and the local skin displacement. We therefore expect spacing-related changes primarily in early sensory regions, i.e. the postcentral gyrus and the posterior occipital cortex, but roughness-related activation changes in higher-order cortical regions, i.e. the insula and the parietal operculum for a roughness estimation task, similar to Kitada et al. (2005); Merabet et al. (2004); Simões-Franklin et al. (2011); Zangaladze et al. (1999). In order to consider all regions that were previously reported in these kinds of tasks, although less consistently, we also included the posterior parietal cortex (Merabet et al., 2007) and the prefrontal cortex (Kitada et al., 2005) in our analyses. Additionally, we considered the inferior temporal cortex to be a candidate region to show an involvement in roughness estimation based on the findings of Sathian et al. (2011). Anatomical regions of interest in the frontal and parietal cortex were added because involvement of these areas is predicted for texture perception and processing by previous literature (Kitada et al., 2005; Ledberg et al., 1995; Merabet et al., 2007; Roland et al., 1998; Stilla and Sathian, 2008; Tremblay et al., 1996; Zangaladze et al., 1999). These included the lateral prefrontal cortex, the primary somatosensory cortex (SI) located on the postcentral gyrus, the operculo-insular cortex as well as the posterior parietal cortex. However, the findings are not completely consistent on left and right-hemispheric contributions. Despite the general idea that the contralateral SI is primarily involved in tactile perception, recent studies also show a contribution of the ipsilateral SI (van Ede et al., 2013). Sathian, Deshpande and Stilla (Sathian et al., 2013) for example reported an involvement of a right postcentral region, ipsilateral to the hand used for stimulus exploration, in a tactile learning experiment. Therefore, for the MVPA analysis bilateral region masks were defined in contrast to focusing only on a single hemisphere. Studies also reported an involvement of temporal and occipital cortical regions in haptic texture perception, but the exact cortical locations are still a matter of debate (Eck et al., 2013a; Merabet et al., 2004; Merabet et al., 2007; Sathian et al., 2011). Therefore, we used an independent visual texture localizer run to identify regions in the temporal and occipital lobe possibly also involved in haptic texture processing. 


\section{METHOD}

\section{Participants}

Thirteen right-handed, healthy volunteers (9 women, 4 men) with normal or corrected-to-normal vision participated in the study. The mean age was 27 years (range: $21-32$ ). Subjects with calluses or (a history of) injuries to the hands were excluded from participation. All participants were graduate and undergraduate students at Maastricht University and received course credit or monetary compensation for their participation in the experiment. The study was approved by the local ethics committee and participants gave written informed consent. One subject (s04) had to be excluded from data analysis due to technical problems resulting in corrupted imaging data.

\section{Stimuli}

Visual Texture Localizer. The stimulus set consisted of 16 texture and 16 shape pictures as well as scrambled versions of the same images. Texture stimuli were grayscale top-view photographs of everyday textures, e.g. abrasive paper, cork, carpet, foam material, wallpaper, Nepal wool and felt. All texture stimuli were the same size. Shape stimuli were grayscale images of abstract 3D shapes, all made of the same material and with approximately the same size. The shape photographs were taken from a slightly tilted front-view perspective to emphasize the three-dimensional characteristic of the objects. Since the images varied slightly in size, a gray circle area with a radius of 200 pixels was created around each picture to match the size of the stimulated visual field across both categories. Scrambled versions of all stimuli were generated by segmenting the image (including the gray circle area) into 64 pixel blocks and rearranging them by generating random permutations of the blocks in the image. In order to control for differences in low-level visual properties between image categories, the luminance and contrast of each image were adjusted to match the average luminance and contrast of the stimulus set. The background of all pictures was set to black (see Figure 1). No analysis was based on the scrambled images in the present study, but these stimuli were included for another functional localizer experiment. 


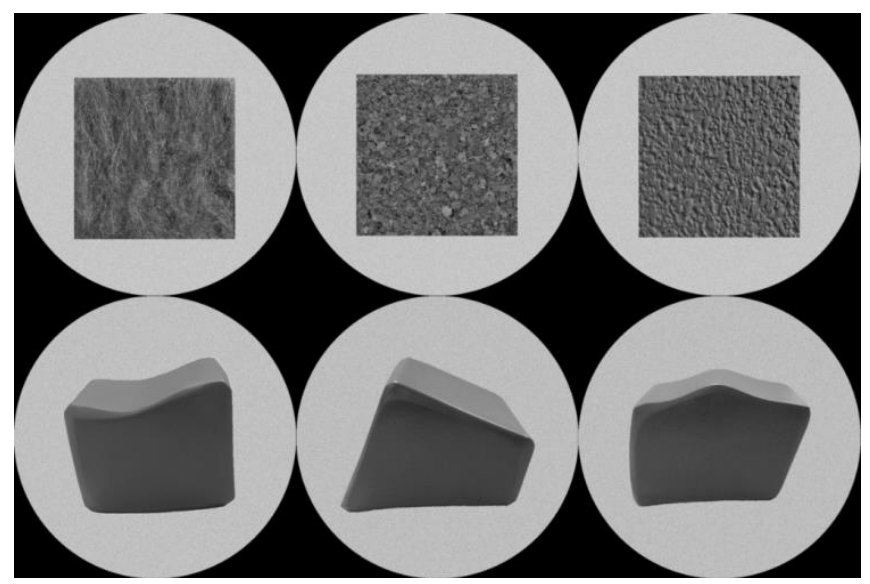

Figure 1. Examples of stimuli used in the visual texture localizer runs. Top row: textures. Bottom row: shapes.

Haptic Texture Exploration Tasks. Haptic stimuli consisted of six 5 x 5 $\mathrm{cm}^{2}$ plastic dot pattern textures. Each dot was $0.8 \mathrm{~mm}$ in diameter and $0.6 \mathrm{~mm}$ in elevation. The dots were arranged non-periodically on the plates. The only characteristic that varied between the textures was the mean center-to-center dot spacing and hence the number of texture elements (see Figure 2). Details about the algorithm used to produce these textures can be found in Eck et al. (2013b). It has been found that haptic roughness perception of dot pattern textures follows approximately an inverted U-shape curve, peaking at about 3-4 $\mathrm{mm}$ inter-element spacing (Connor et al., 1990; Eck et al., 2013b; Gescheider et al., 2005; Merabet et al., 2004; Merabet et al., 2007). For density and distance perception on the other hand a linear function can be expected with increasing inter-dot spacing (Eck et al., 2013a; Merabet et al., 2004). Using the same stimulus set to sample the roughness and density curve at different curve sections allows us to identify cortical responses that vary with perceived stimulus characteristics, rather than purely presenting differences in the physical stimulus properties (i.e. inter-element spacing). The inter-dot spacings of the textures selected for this study were $1.50 \mathrm{~mm}, 2.25 \mathrm{~mm}, 3.50 \mathrm{~mm}, 4.50 \mathrm{~mm}$, $5.50 \mathrm{~mm}$ and $8.25 \mathrm{~mm}$. Figure 3 illustrates the locations of these textures on the perceived roughness and density curve as hypothesized based on previous behavioral studies (Eck et al., 2013b; Merabet et al., 2004). 


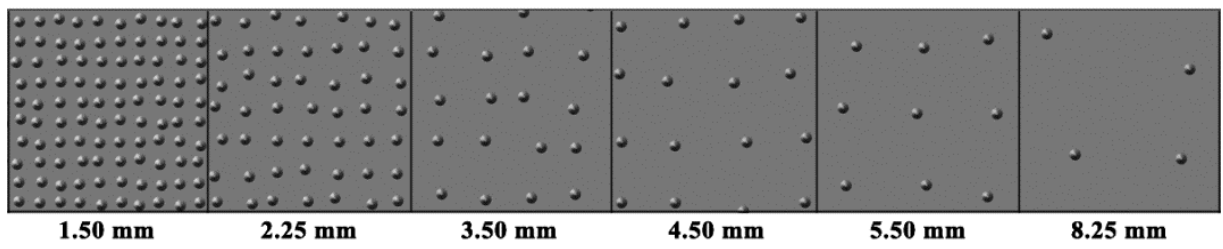

Figure 2. Magnified excerpts from the dot pattern textures used in the haptic experimental runs.

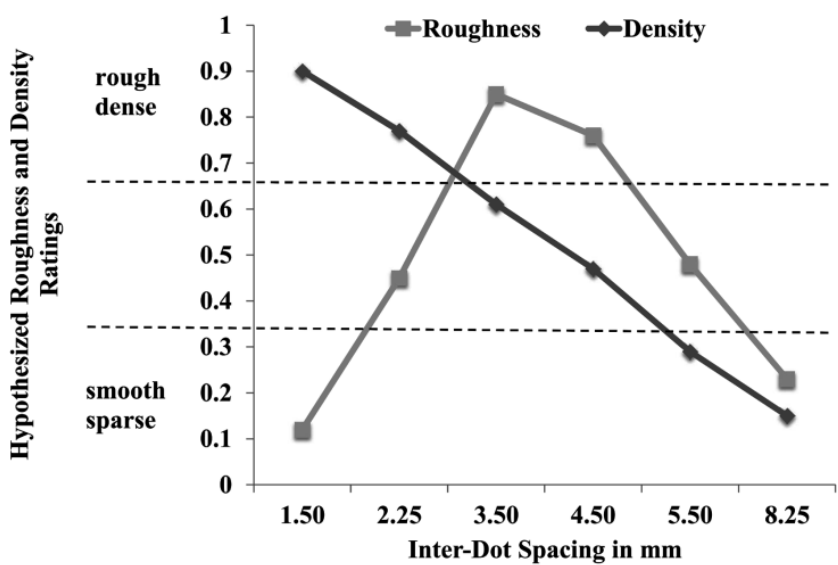

Figure 3. Schematic representation of the stimulus space with regard to the expected roughness and density judgments.

\section{Experimental setup}

We used a similar experimental setup as published previously (Eck et al., 2013a). All dot pattern stimuli were placed on a circular wooden board, covered by another plate with a rectangular cutout that assured that subjects could touch only a single texture. The entire presentation device was placed over the thighs of the participant and was attached to the scanner table. The right hand of the subject, meant to explore the textures, was placed on top of the cover-plate, while the left hand, that operated the button box, lay next to the participant's legs. Both arms were supported by foam padding to reduce movement during the scanning. The presentation of the haptic stimuli was controlled by the experimenter who was standing next to the scanner bore. Visual cues were displayed centrally on a black background. The images were projected onto a rear-projection screen at the end of the scanner bore and subjects viewed the stimuli via a mirror mounted to the head-coil. Stimulus timing and presentation was controlled by Presentation $\AA$ software (Neurobehavioral Systems, Inc., Albany, CA, USA). 


\section{Experimental procedure}

Visual Texture Localizer. Texture and shape images as well as scrambled versions of both categories were presented in separate blocks of eight, each block lasting $16 \mathrm{~s}$. The four different block types (i.e., texture, shape, scrambled texture and scrambled shape) were interleaved with $16 \mathrm{~s}$ blocks of rest. Each image was presented three times during the independent localizer run.

Both stimulus presentation within category blocks and the order of blocks was randomized. For task blocks, subjects were informed of the category of the next block (i.e. 'Scrambled', 'Texture' or 'Shape') by an auditory cue via headphones $700 \mathrm{~ms}$ before block onset. The beginning of the rest interval was cued by the word 'Rest'. After the last image in each texture and shape block, a question was displayed asking whether the last item presented was already presented before in the same block. This task was chosen to ensure subjects' attention to the images. Subjects responded by pressing one of two buttons on a response box attached to their left hand. For the scrambled texture and shape blocks subjects were instructed to passively view the images. During picture presentation a red fixation dot was displayed in the center of the screen, while a white fixation dot was presented during rest blocks. Participants were asked to fixate on these dots during the localizer run.

Haptic Texture Exploration Tasks. Subjects were informed that they would be presented with one haptic texture at each trial. In 'Rating' runs participants had to judge the perceived roughness or spatial density after stimulus presentation, in the 'No-Estimation' runs subjects were told to focus on the haptic exploration of the textures. The experimenter stressed the importance of focusing on the mere stimulus exploration in the No-Estimation runs as in contrast to judging the perceived stimulus properties in the Rating runs. Participants explored the textures by sweeping four times with their right index-, middle- and ring finger (simultaneously) across the surface. After this exploration movement subjects placed their hand back in the resting position on top of the wooden cover plate. In the Rating runs the haptic exploration interval $(4 \mathrm{~s})$ was followed by a jittered delay $(5-9 \mathrm{~s})$ and a rating time window $(3 \mathrm{~s})$. These task trials alternated with rest intervals of $12-14 \mathrm{~s}$ (jittered). During the rating time window, subjects were asked to judge the perceived roughness or density of the previously explored dot pattern on a 400 pixels visual analogue scale (VAS) ranging from very smooth / sparse to very rough / dense. The slider of the VAS was moved to the left or to the right by pressing two buttons on a fiber-response box attached to the subject's left hand. In the beginning of the rating interval the slider was always positioned in the middle of the scale. In the No-Estimation runs exploration was immediately followed by a rest time window (see Figure 4). Auditory cues, delivered via headphones in the intertrial-intervals (rest), instructed the experimenter to turn the haptic presentation device to the correct stimulus for the next trial. Subjects in turn used visual cues to identify the correct trial phase. A red dot presented in the center of the screen 
indicated the haptic exploration interval, a blue dot marked the delay period and a white dot presented the rest interval. Density and roughness conditions were blocked in the Rating runs and the words 'Roughness' and 'Density', displayed on the screen in the beginning and the middle of the runs, indicated the task for the upcoming trials. The labeling of the endpoints of the VAS was adapted to the task condition. The order of the task blocks was varied across runs and was changed for all participants. Each of the six dot patterns was repeated 15 times in each task condition (roughness vs. density rating vs. no-estimation) over the course of the fMRI experiment.

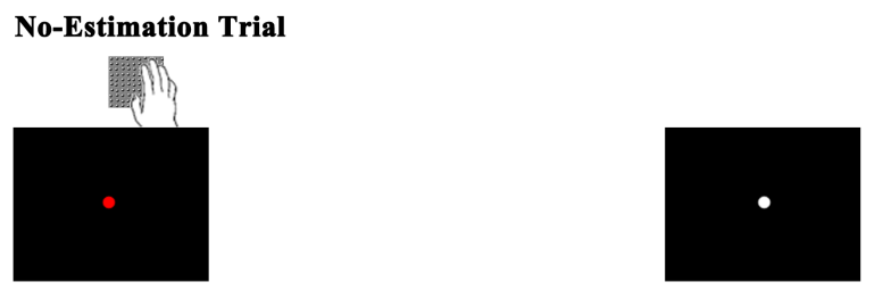

\section{Roughness / Density Rating Trial}
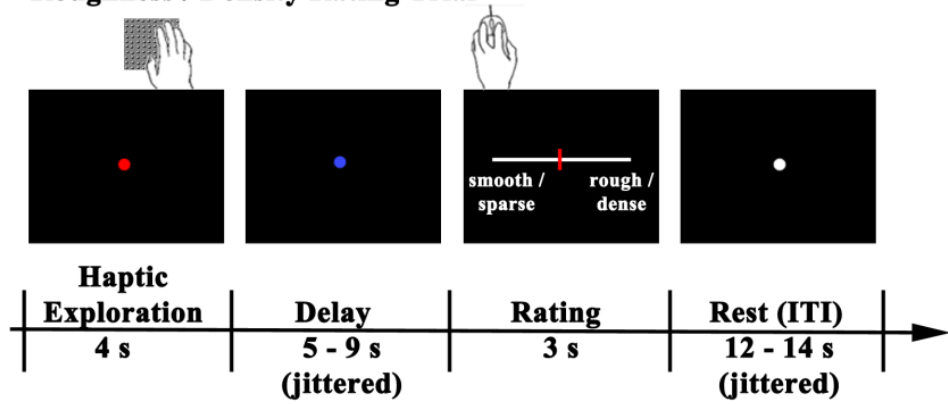

\section{No-Estimation Runs}

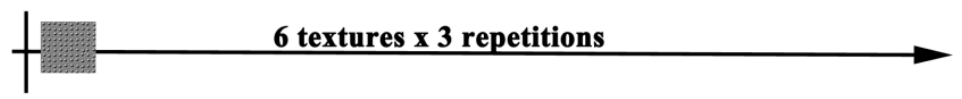

\section{Roughness / Density Rating Runs}

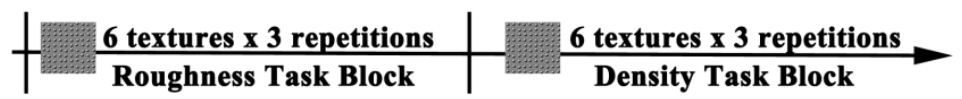

Figure 4. Illustration of the experimental design for the Rating and the No-Estimation runs. ITI $=$ inter trial interval.

Each subject participated in three experimental sessions on three different days. In the first experimental session subjects were familiarized with the Rating and No-Estimation runs in a mock scanner. This was done to minimize movement artifacts in the following scanning sessions. Subjects practiced the exploration movement and were introduced to the VAS and the sensitivity of the response button box. The same stimuli as in the fMRI 
experiment were used in the practice session to familiarize participants with the stimulus range; each stimulus was repeated four times within the three task conditions. The practice session lasted until subjects were comfortable with the tasks and until the movement was experienced by the subject as effortless, it was synchronized with the duration of the exploration interval and all other motion was reduced to a minimum. The duration of the mock scanner session was approximately 30 minutes for all subjects. The second session comprised the visual texture localizer run as well as five No-Estimation runs, each 170 volumes long and therefore lasting about 6 minutes. In the third session participants completed the five Rating runs, each 510 volumes long, lasting 17 minutes. Data for the Rating and No-Estimation runs were acquired in different sessions to keep the scanning time in each session in a reasonable timeframe.

\section{Data acquisition}

Data were acquired with a 3 Tesla Siemens Allegra MR head scanner equipped with a standard quadrature birdcage coil at the Maastricht Brain Imaging Center (Maastricht, The Netherlands). In both scanning sessions a standard anatomical T1-weighted data set (MPRAGE) was acquired that covered the whole brain $\left(\mathrm{TR}=2250 \mathrm{~ms}, \mathrm{TE}=2.6 \mathrm{~ms}, \mathrm{FA}=9^{\circ}\right.$, matrix: $256 \mathrm{x}$ 256, voxel size: $1 \times 1 \times 1 \mathrm{~mm}^{3}$, 192 slices). In the first scanning session this was followed by the five No-Estimation runs. After a short break the first scanning session was finalized with the visual texture localizer run. In the second scanning session the anatomical image acquisition was followed by the five Rating runs. All functional images were obtained with a T2*-weighted echoplanar imaging $(\mathrm{EPI})$ sequence $(\mathrm{TR}=2000 \mathrm{~ms}, \mathrm{TE}=30 \mathrm{~ms}$, flip angle $(\mathrm{FA})=$ $90^{\circ}$, matrix: $64 \times 64$, field of view (FOV): $224 \times 224$, slice thickness $=3.5 \mathrm{~mm}$, 32 slices covering the whole brain, no gap).

\section{Data analysis}

Trials in which subjects either missed the exploration or the rating interval of the haptic stimulus were removed from behavioral and fMRI data analysis. One subject missed 4 out of 90 trials in the density rating task; in all other subjects the number of missed trials did not exceed two for any of the task conditions.

Behavioral Data Analysis. In order to evaluate the variability of the ratings across subjects and repetitions, the mean roughness and density curve was assessed for each subject (see Figure 5). For the majority of subjects the mean roughness and density curve was comparable to the results of our previous behavioral study (Eck et al., 2013b). However, for some of the participants perceived roughness did not decrease as much for wider inter-dot spacing as 
was hypothesized based on the literature. This high inter-individual variability was not expected, but the minimally sampled stimulus space with six stimuli only, versus 29 stimuli in our earlier study might have influenced the results. Subjects that did not show a decrease in perceived roughness below the middle of the VAS scale ( 0 ) for wide dot spacing textures had to be excluded from data analysis, because an unambiguous categorization of stimulus spacings to different roughness classes (independent from perceived density) was not possible. Therefore, participants $\mathrm{s} 05, \mathrm{~s} 06, \mathrm{~s} 09, \mathrm{~s} 13$ are not considered in the following descriptions.

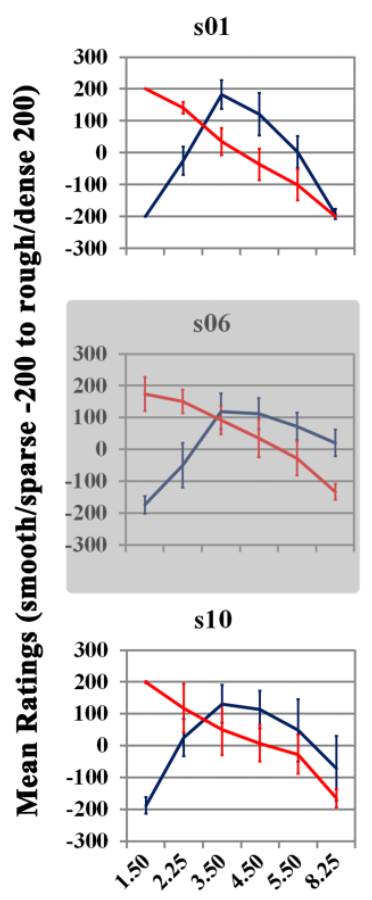

-Mean Roughness -Mean Density
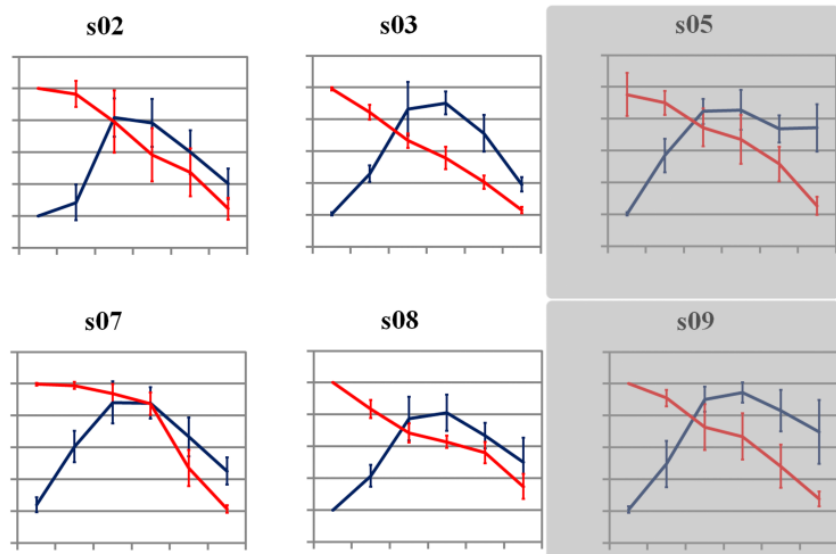

s09

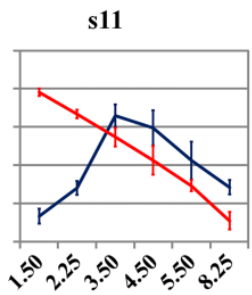

s12

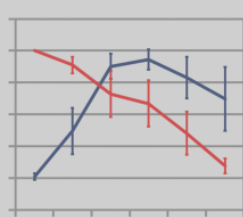

Average Inter-Dot Spacing in mm

Figure 5. Mean density and roughness ratings for all dot pattern textures averaged over all repetitions and separately for all subjects. Error bars represent the standard deviation. Gray highlighted subjects were excluded from all subsequent analyses.

For multi-voxel pattern classification, we grouped the fMRI trial data into categories. Based on the mean roughness and density ratings, trial categorization to a low and high density/roughness class was performed as follows. For the high density and roughness class we selected the two dot pattern textures with the highest mean ratings. Hence, for all subjects the trials with $1.50 \mathrm{~mm}$ and $2.25 \mathrm{~mm}$ inter-dot spacing were categorized as high density, while the 3.50 and $4.50 \mathrm{~mm}$ stimuli were grouped to a high roughness class. For the low density class we selected the two stimuli with the lowest mean density 
ratings over all repetitions, i.e. 5.50 and $8.25 \mathrm{~mm}$ inter-dot spacing for all subjects. For the low roughness class we combined this rating criterion with a spacing criterion. We selected the stimulus with the lowest roughness rating in the wide inter-dot spacing range $(4.50 \mathrm{~mm}-8.25 \mathrm{~mm})$, i.e. $8.25 \mathrm{~mm}$ for all subjects and the stimulus with the next lowest roughness rating in the close inter-dot spacing range $(1.50 \mathrm{~mm}-3.50 \mathrm{~mm})$, i.e. $1.50 \mathrm{~mm}$ for 5 out of 8 subjects and $2.25 \mathrm{~mm}$ for 3 out of 8 subjects. The combination of the rating and spacing criterion for the low roughness category was chosen to avoid a complete overlap with the high density category. This ensures that an above chance classification of the high and low roughness category is confounded neither with stimulus spacing nor with perceived density.

To ensure unambiguous category representations, individual trials with ratings that could not be clearly assigned to either the high or low category (border trials) were excluded from data analysis. Trials in the No-Estimation runs with the same inter-dot spacing stimuli as in the high and low density categories of the Rating runs were grouped in a close and a wide spacing category. This was done to rule out those cortical regions showing above chance classification of density classes in the Rating runs which merely reflect the physical stimulus characteristics. An overview of trial categorization can be found in Table 1. Task classification, i.e. trials in the roughness task blocks versus density task blocks, was based on all dot pattern textures. The rationale of this analysis was to investigate the effect of task instructions independently of the objective stimulus space.

fMRI Data Preprocessing. Functional and anatomical data were analyzed with BrainVoyager QX 2.4.2 (Brain Innovation, Maastricht, The Netherlands). The first two scans of each functional run were discarded to allow for T1 equilibration. Preprocessing of the functional data included slice scan time correction, intra-session alignment to detect and correct for small head movements by rigid body transformations, temporal filtering removing linear trends and non-linear temporal frequencies of 3 or less cycles per run. No subject had to be removed because of excessive head motion. Functional images of all runs were co-registered to the anatomical volumes of the respective subject and scanning session and transformed into Talairach space resulting in an interpolated functional voxel size of $3 \times 3 \times 3 \mathrm{~mm}^{3}$. Based on the T1weighted anatomical data sets individual surface reconstructions of the cortical sheet were created employing an automatic white-gray matter segmentation approach. Segmentation errors of the automatic procedure were manually postcorrected. These individual cortical surfaces were aligned to a moving target group average by using a curvature-driven cortical mapping approach (Frost and Goebel, 2012; Goebel et al., 2006). The results were used to create an average cortex representation of all subjects. Furthermore, the mapping parameters from cortex-based alignment (CBA) were used to align individual statistical maps to the group average cortical surface. 
Table 1. Summary of trial categorizations to Roughness (R), Density (D), Spacing (S) and Task classes.

\begin{tabular}{|c|c|c|c|c|c|c|c|c|c|c|}
\hline \multirow{4}{*}{$\begin{array}{l}\text { Sub- } \\
\text { ject }\end{array}$} & \multirow{2}{*}{\multicolumn{3}{|c|}{$\begin{array}{l}\text { Dot Pattern } \\
\text { Spacings Grouped } \\
\text { in High/High/Close } \\
\text { Category }\end{array}$}} & \multirow{2}{*}{\multicolumn{3}{|c|}{$\begin{array}{l}\text { Dot Pattern } \\
\text { Spacings Grouped } \\
\text { in Low/Low/Wide } \\
\text { Category }\end{array}$}} & \multirow{3}{*}{\multicolumn{4}{|c|}{$\begin{array}{l}\text { Number of Trials in } \\
\text { Categories } \\
\text { (High/High/Close/R- } \\
\text { Task, Low/Low/Wide/D- } \\
\text { Task) }\end{array}$}} \\
\hline & & & & & & & & & & \\
\hline & & & & & & & & & & \\
\hline & $\mathrm{R}$ & D & $\mathrm{S}$ & $\mathrm{R}$ & D & $S$ & $\mathrm{R}$ & $\mathrm{D}$ & $\mathrm{S}$ & Task \\
\hline \multirow[t]{2}{*}{ s01 } & 3.50 & 1.50 & 1.50 & 1.50 & 5.50 & 5.50 & $29 *$ & 30 & $30 *$ & R: $89 *$ \\
\hline & 4.50 & 2.25 & 2.25 & 8.25 & 8.25 & & 30 & $29 *$ & 30 & D: 89 \\
\hline \multirow[t]{2}{*}{ s02 } & 3.50 & 1.50 & 1.5 & 2.25 & 5.50 & 5.50 & $27 *$ & 29 & $29 *$ & R: $84^{*}$ \\
\hline & 4.50 & 2.25 & 2.2 & 8.25 & 8.2 & 8. & 28 & $28 *$ & 30 & D: 86 \\
\hline \multirow[t]{2}{*}{ s03 } & 3.50 & 1.50 & 1.5 & 1.50 & 5.50 & 5.5 & $29 *$ & $30 *$ & $30 *$ & R: $88^{*}$ \\
\hline & 4.50 & 2.25 & 2. & 8.25 & 8. & & 29 & 30 & 30 & D: 90 \\
\hline \multirow[t]{2}{*}{ s07 } & 3.50 & 1.50 & 1.5 & 1.50 & 5.50 & 5. & $28 *$ & 29 & $29 *$ & R: $86^{*}$ \\
\hline & 4.50 & 2.25 & 2.2 & 8.25 & 8.25 & 8. & 28 & $28 *$ & 30 & D: 87 \\
\hline \multirow[t]{2}{*}{ s08 } & 3.50 & 1.50 & 1.5 & 2.25 & 5.50 & 5.5 & $27 *$ & $28 *$ & $30 *$ & R: $84^{*}$ \\
\hline & 4.50 & 2.25 & 2.25 & 8.25 & 8.25 & 8.2 & 27 & 28 & 30 & D: 86 \\
\hline \multirow[t]{2}{*}{ s10 } & 3.50 & 1.50 & 1.50 & 1.50 & 5.50 & 5.5 & 27 & 28 & $29 *$ & R: $83^{*}$ \\
\hline & 4.50 & 2.25 & 2.25 & 8.25 & 8.25 & 8.25 & $26^{*}$ & $27 *$ & 30 & D: 85 \\
\hline \multirow[t]{2}{*}{ s11 } & 3.50 & 1.50 & 1.50 & 2.25 & 5.50 & 5.50 & $27 *$ & $30 *$ & $30 *$ & $\mathrm{R}: 85^{*}$ \\
\hline & 4.50 & 2.25 & 2.25 & 8.25 & 8.25 & 8.25 & 28 & 30 & 30 & D: 90 \\
\hline \multirow[t]{2}{*}{$\mathrm{s} 12$} & 3.50 & 1.50 & 1.50 & 1.50 & 5.50 & 5.50 & $29 *$ & 30 & $30 *$ & $\mathrm{R}: 87^{*}$ \\
\hline & 4.50 & 2.25 & 2.25 & 8.25 & 8.25 & 8.25 & 29 & $29 *$ & 30 & D: 89 \\
\hline
\end{tabular}

Note. All dot pattern spacings were used in the Roughness - Density Task Classification.

* number of trials per class used for both categories in the multi-voxel pattern analysis.

Univariate Data Analysis. For group analysis a whole-brain fixedeffects regression analysis (FFX) was performed because of the sample size of $\mathrm{N}=8$ remaining after removal of participants with corrupted data (s04) and similar roughness and density rating curves (s05, s06, s09, s13). Three different general linear models (GLM) were used to address different aspects of the research question.

The first model comprised seven predictors. All spacing trials of the roughness task condition were grouped to one predictor and the same was done for all trials in the density task blocks. These two predictors were defined for each of the three trial intervals, i.e. haptic exploration, delay and rating interval. The presentation of the visual cues 'Roughness' and 'Density' during the experimental runs was modeled as the seventh predictor. One contrast was computed for that model asking for differences between the roughness and density task during haptic exploration, irrespective of the stimulus characteristics ('Exploration: roughness $\neq$ density'). 
In a second model 19 task-related predictors were defined based on the trial categorization in the Rating runs as explained above. The predictors represented the four categories high/low roughness/density as well as two additional predictors containing all trials in the two task conditions that could not be assigned to any of the other four categories. These predictors were defined for each trial phase and the last predictor accounted for the visual cue. Two different contrasts were computed for the haptic exploration interval of that model, namely 'Exploration: high roughness $\neq$ low roughness' and 'Exploration: high density $\neq$ low density'.

The third model is based on the No-Estimation runs. Three predictors were defined, representing trial categorization with regard to the objective stimulus characteristics, i.e. inter-dot spacing of the textures. Two predictors contained the close and wide inter-dot spacing trials while a third predictor represented the remaining dot pattern trials. The contrast computed for that model compared close and wide spacing trials ('Exploration: close spacing $\neq$ wide spacing').

All predictor time courses were convolved with a two-gamma hemodynamic response function (HRF) to account for the hemodynamic response delay. The voxel-threshold for statistical significance was first set to $\alpha$ $=0.005$ uncorrected and all statistical contrast maps were then corrected for multiple comparisons using cluster-size thresholding (Forman et al., 1995; Goebel et al., 2006) with a cluster-level false positive rate of $\alpha=0.05$.

Multivariate Data Analysis. In order to reduce the number of initial features for multivariate data analysis, anatomical and functional masks were defined on the individual cortical meshes.

Anatomical cortex masks. All anatomical regions of interests were delineated on the individual cortex meshes. The posterior parietal cortex (PPC) comprised the superior parietal and inferior parietal lobule - separated by the intra-parietal sulcus (Culham and Valyear 2006). The second region including the primary somatosensory cortex (SI) was defined based on the location of the postcentral gyrus (PoCG) and the central sulcus (CS) (Sanchez-Panchuelo et al., 2010; Stringer et al., 2011). The third operculo-insular region (OpIns cortex) circumscribed the insular cortex and the parietal operculum on which the human secondary somatosensory cortex (SII) is located (Eickhoff et al., 2007; Eickhoff et al., 2008; Mazzola et al., 2012). The last anatomical region of interest was the frontal/prefrontal cortex. The approximate boundaries of this cortical patch were defined as follows. Posteriorly the boundary was the inferior precentral sulcus and superiorly the superior frontal gyrus. The inferior boundary was represented by the inferior frontal gyrus and anteriorly the lateral part of the frontopolar prefrontal cortex was included. The extent of the anatomical masks was intentionally slightly bigger than in conventional studies (e.g. Goulas et al., 2012) to account for inter-individual differences in anatomical - functional correspondence. 
Functional cortex masks. Based on the functional localizer run, a regression analysis was computed with six predictors: Texture block, Shape block, Scrambled texture block, Scrambled shape block, Question and Response. In order to identify regions in the temporal and occipital lobe that show a tendency to respond stronger to texture than to shape images within our sample of $\mathrm{N}=8$, we ran the above described general linear model first at the FFX level, with a temporal and occipital lobe mask for all participants, comparing texture and shape blocks (Texture block > Shape block). We identified a bilateral posterior occipital cortex cluster (pOCC) and a bilateral cluster in the ventral temporal cortex (VTC) bordering the fusiform gyrus (FG) and the collateral sulcus $(\mathrm{CoS})$ (see Figure $6(\mathrm{~A})$ ). Afterwards we ran the same model and contrast on the single subject level, identifying activation clusters in each individual subject. Statistical maps were thresholded as described above. The number of voxels in the resulting clusters varied substantially between individuals. To reduce size differences between the anatomical and functional cortex masks, we defined the four subject-specific cortex masks in the temporal and occipital lobe as 800 voxel regions around the cluster-specific peak voxels in the individual Texture versus Shape contrast. Significant activation clusters were identified in 7 out of 8 subjects in the left hemisphere of the posterior occipital cortex; this was also true for 6 out of 8 participants in the right pOCC and the left VTC. Above threshold activations were however only present in 3 out of 8 subjects in the right VTC. For participants in which any of the four regions were not present in the thresholded individual contrast maps, the subject-specific peak voxel within the respective FFX cluster was determined and used as the origin of the mask definition. A summary of the subject-specific peak voxels can be found in Table 2 .

Table 2. Individual peak voxels in the occipital and temporal lobe of the contrast maps: Texture block > Shape block. Talairach coordinates of the peak voxels with the respective t-values.

\begin{tabular}{|c|c|c|c|c|c|c|c|c|}
\hline \multirow{2}{*}{$\begin{array}{l}\text { Sub- } \\
\text { ject }\end{array}$} & \multicolumn{2}{|l|}{ right pOCC } & \multicolumn{2}{|l|}{ left pOCC } & \multicolumn{2}{|l|}{ right VTC } & \multicolumn{2}{|l|}{ left VTC } \\
\hline & $\mathrm{x}, \mathrm{y}, \mathrm{z}$ & $\mathrm{t}$ & $\mathrm{x}, \mathrm{y}, \mathrm{z}$ & $\mathrm{t}$ & $\mathrm{x}, \mathrm{y}, \mathrm{z}$ & $\mathrm{t}$ & $\mathrm{x}, \mathrm{y}, \mathrm{z}$ & $\mathrm{t}$ \\
\hline s01 & $17,-98,0$ & 5.8 & $-25,-92,-6$ & 8.2 & $26,-44,-15$ & 5.6 & $-34,-47,-15$ & 6.2 \\
\hline $\mathrm{s} 02$ & $20,-101,0$ & 7.5 & $-13,-92,0$ & 10.2 & $29,-56,-9$ & $4.3^{*}$ & $-25,-59,-12$ & 6.8 \\
\hline s03 & $8,-92$ & 3.9 & $5,-21$ & 4.6 & $1,-18$ & $3.6^{*}$ & $53,-18$ & $2.6^{*}$ \\
\hline s07 & $14,-83,-6$ & 6.9 & $-25,-92,-9$ & 6.8 & $20,-50,-12$ & 5.8 & $-28,-59,-18$ & 5.7 \\
\hline s08 & $17,-95,-6$ & 5.7 & $-16,-92,-3$ & 7.1 & $24,-53,-15$ & $3^{*}$ & $-19,-59,-24$ & 5.9 \\
\hline s10 & $11,-95,-6$ & $3.6^{*}$ & $-16,-92,-15$ & 5.8 & $23,-38,-15$ & $4.3^{*}$ & $-25,-35,-18$ & 5.5 \\
\hline s11 & $14,-92,-6$ & $3.2 *$ & $-25,-89,-3$ & $3.6^{*}$ & $32,-56,-9$ & $3.1^{*}$ & $-25,-56,-15$ & $2.4 *$ \\
\hline s12 & $8,-92,-21$ & 4.7 & $-13,-101,6$ & 5.4 & $29,-26,-18$ & 5.3 & $-28,-65,-15$ & 4.8 \\
\hline
\end{tabular}

$*$ individual peak voxels in FFX clusters. 


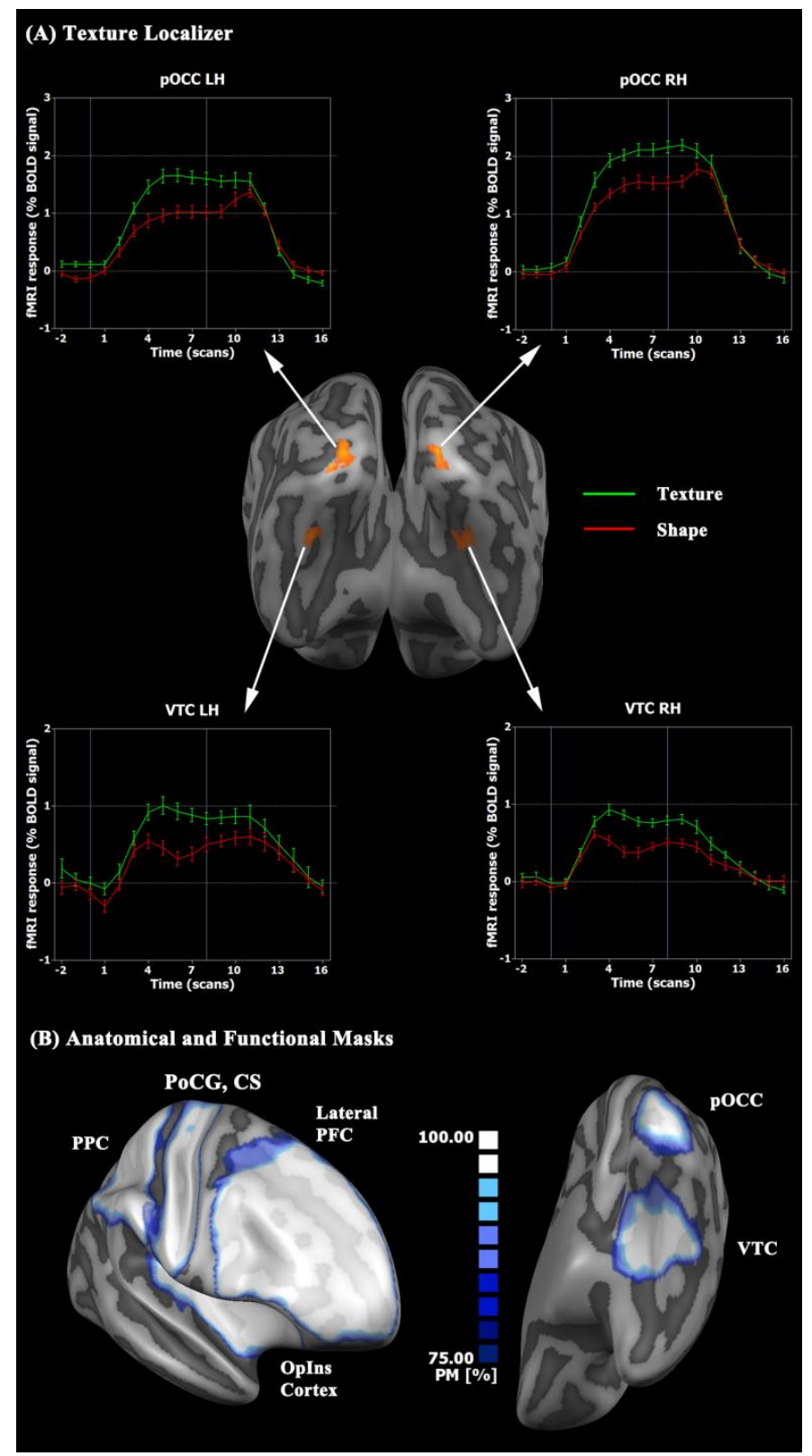

Figure 6. Anatomical and functional masks. (A) FFX contrast map of the texture localizer: Texture block $>$ Shape block overlaid on the average cortex reconstruction of all subjects. (B) Probability map of individually defined cortex masks illustrated exemplarily on the average cortical sheet of the right hemisphere. Only vertices that are represented in 6 out of 8 subjects' masks are color coded. $\mathrm{PPC}=$ posterior parietal cortex, $\mathrm{PoCG}=$ postcentral gyrus, $\mathrm{CS}=$ central sulcus, $\mathrm{PFC}=$ prefrontal cortex, $\mathrm{pOCC}$ $=$ posterior occipital cortex, $\mathrm{VTC}=$ ventral temporal cortex. 
The location of the bilateral masks is illustrated exemplarily on the right average cortex representation in Figure 6 (B). Due to inter-individual differences in the anatomy, no perfect overlap of individual cortex masks and the subject-averaged folding pattern of the cortical sheet can be expected even after macro-anatomical alignment (CBA).

Four different models were considered for multivariate pattern analysis on single subject level, separately for all six individual cortex masks: (1) roughness vs. density haptic exploration trials (Rating runs), (2) high vs. low density haptic exploration trials (Rating runs), (3) high vs. low roughness haptic exploration trials (Rating runs) and (4) close vs. wide spacing haptic exploration trials (No-Estimation runs). Mask voxels with a mean raw signal intensity below 70 and a standard deviation below 1 were excluded from data analysis to reduce noise in the data. Single trial estimation was performed by creating separate multi-voxel response patterns for all trials, subjects, region masks, and models. The response pattern of a single trial was estimated by extracting the average signal for TR 2, 3 and 4 after onset of the haptic exploration interval. This signal was transformed to percent signal change by dividing it by the mean baseline signal of TR $-2,-1,0$ with regard to stimulus onset and multiplying it by 100 . The extracted single-trial responses of each voxel were normalized by subtracting the mean and dividing by the standard deviation of the training trials of the respective voxel. Depending on the trial categorization scheme of the four different models, trial response patterns were labeled according to the classes described above (low/high density/roughness/spacing or density versus roughness trials irrespective of the stimulus differences). See Table 1 for the number of trials per class and subject. In order to equalize the number of trials per class in each model, we used as many trials for analysis as were available in the class with the lowest number of trials (marked in Table 1 with a*). These trials were selected randomly. However, within a subject and model the same number of trials was used for both classes.

For analysis of the multi-voxel patterns we combined linear support vector machines (SVMs) with an iterative multivariate voxel selection approach - recursive feature elimination (RFE) (De Martino et al., 2008) - in order to remove non-informative voxels that can degrade classification performance. For classifier training we used a training set containing 20 of all category trials for the roughness, density and spacing model and 60 trials for the task model. The test set used for assessing the performance and generalization ability of the classifier, consisted of the remaining trials. The RFE algorithm started with the full number of voxels in the masks. At each RFE iteration 2/3 of the training data was used to train a least square SVM (1s-SVM) (Suykens et al., 2002). The classification model (i.e. weights) was used to create discriminative maps that indicate the importance of each voxel to class discrimination. There were 20 
(A) Mask Definition

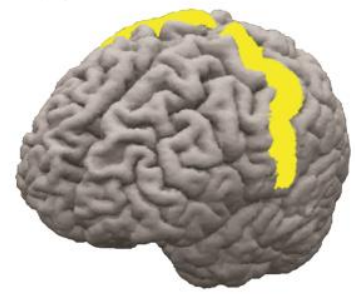

(C) RFE

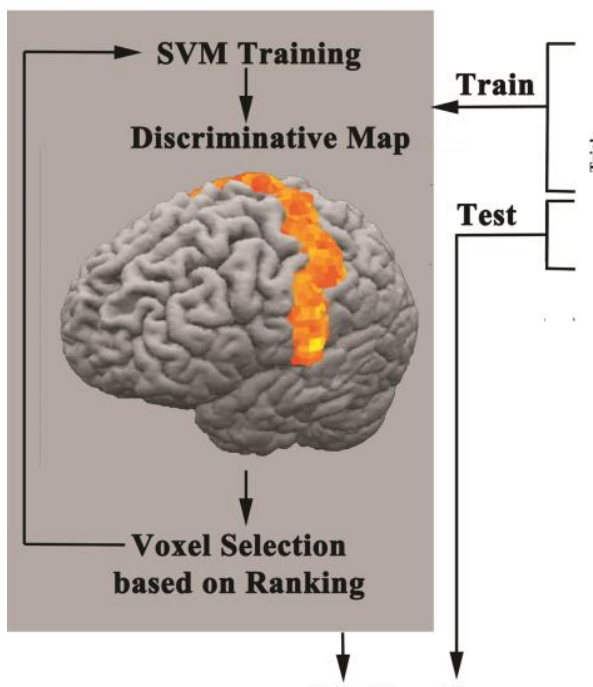

(D) Classifier
(B) Feature Extraction
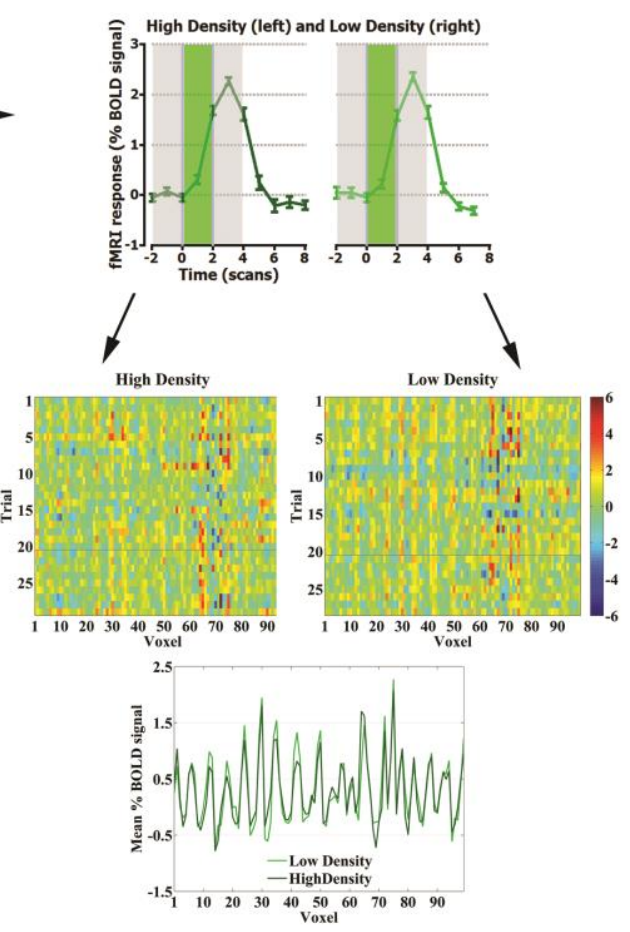

Accuracy

Figure 7. Overview of the multivariate data analysis illustrated exemplarily on the high/low density classification within the postcentral gyrus mask of a single subject. (A) fMRI time course data were extracted from all voxels within the defined mask. (B) Features were defined for all trials and for each density class separately by selecting the mean signal of the second, third and fourth scan after stimulus onset, visualized by the second gray segment in the upper row plots. This stimulus-related signal was percentnormalized using the baseline signal, represented by the first gray segment in the same plots. The green demarcated time interval represents the stimulus duration of $4000 \mathrm{~ms}$. The lower two plots show the multi-voxel response patterns of all trials within both categories for 100 out of the initial 1800 postcentral gyrus mask voxels of that participant. The plot below represents the averaged pattern over all trials for the same 100 voxels, separately for both density classes. (C) The RFE approach was used to reduce the number of voxels and select the pattern with the most discriminative information. (D) An independent set of test trials was used to test the generalization performance of the classifier.

iterations, each repeating this procedure with a different subset of training trials. Each RFE voxel selection level was concluded by averaging the discriminative weights for each voxel over the 20 iterations, ranking the average discriminative 
weights and discarding voxels amongst the lowest $6 \%$ corresponding to the smallest ranking. The number of RFE voxel selection levels was adapted to the initial mask size and ranged from 61 to 84 .

The entire RFE procedure was performed in 20-fold cross-validation. See Figure 7 for a summary of the multivariate analysis approach. The final accuracy at each RFE voxel selection level was computed as the mean over these 20 cross-validations. The classification performance reported below represents the average maximum accuracy across all RFE voxel selection levels. A two-step procedure was used to assess whether the difference between the actual accuracy and the empirical chance level was significantly above 0 across subjects for a specific model and region. In a first step permutation tests were performed for each subject and region of interest (ROI). We used exactly the same feature extraction and SVM-RFE combination approach as explained above, but labels were randomly assigned to trial response patterns and the permutation was repeated 100 times. The final accuracy value of a single permutation was defined as the maximum prediction accuracy across all RFE steps, averaged over all cross-validations. The accuracies of all 100 permutations represent the permutation distribution of the chance level. The medians of these subject-specific null-distributions were used in the second step employing an exact permutation test. Assuming that the null hypothesis is true, namely that the actual accuracies across subjects are at chance level, multiplying any of the subjects' difference (actual accuracy -median permutation accuracy) by -1 will have no effect on the correctness of the null hypothesis. Considering a sample size of 8 subjects, there are $2^{\wedge} 8$ (256) permutations possible. These permutations constitute an estimate of the statistical distribution of the random effects (RFX) null hypothesis. Above chance classification across subjects was assumed when the mean of the subject-specific differences was at or above the 95th percentile of the null distribution.

In order to visualize regions that are involved in task or roughness/density/spacing classification across subjects, single subject maps were combined to a discriminative group map. For each subject a map was created showing voxels that survived at least $25 \%$ of the RFE voxel selection levels. These maps were sampled on the reconstructed cortex of each subject and aligned to the group-average cortex mesh using the CBA parameters. In order to combine the single subject maps to a group map only vertices were color coded that were presented in at least 6 out of the 8 single subject maps. The group-average map was thresholded by applying a cluster size threshold of $25 \mathrm{~mm}^{2}$. This approach was applied for each model and region mask. 


\section{RESULTS}

\section{Univariate data analysis}

General Task Networks. Haptic exploration of the texture stimuli evoked BOLD responses in a wide cortical network consistently for all three task conditions, i.e. roughness estimation, density estimation and exploration without a cognitive task. This network included early and higher-order visual, motor and somatosensory regions, as well as the cingulate, prefrontal and the posterior parietal cortex (see Figure 8 (A)). There are no obvious differences in the extent of the network between roughness and density task instructions.

As expected, the activated network looks slightly different when subjects are instructed to explore the textures without a rating task. The lateral prefrontal cortex as well as medial parietal and occipital regions appear to play a less prominent role in haptic texture exploration when no cognitive evaluations of the textures are required. However, the purely perceptual task engages a network that is slightly more extended in lateral visual and temporal cortex regions as compared to texture evaluations. Note that statistical comparison of the exploration period in the Rating and the No-Estimation runs was not possible, because these data were acquired in different experimental sessions. The above described differences are the result of overlaying the statistical maps of the different runs on a single cortical mesh.

Effects of Task Instructions. A direct statistical comparison of the haptic exploration interval in the density and roughness estimation task confirmed the observations described above, namely no significant difference in the univariate activation maps for both tasks.

Effects of Perceived Density. The comparison of haptic exploration trials producing high versus low density ratings revealed significantly lower activations for high density trials (close stimulus spacing) in the ipsilateral PoCG extending into the postcentral sulcus (PoCS) (peak voxel: $t(7)=-4.43, p$ $<.001$ ), including Brodmann's area 1 (BA 1). An event-related average showed that this effect represents differences above baseline level (see Figure 8 (B)). No other brain areas showed differential activations for the same contrast in the univariate data analysis.

Effects of Perceived Roughness. No above threshold activations were found for the comparison of trials that were grouped into low and high roughness categories based on the trial-specific roughness ratings of the participants.

Effects of Stimulus Spacing. Employing the inter-dot spacing of the textures in the No-Estimation runs for trial grouping did not yield any significant differences in the activation levels between wide and close spacing trials. 


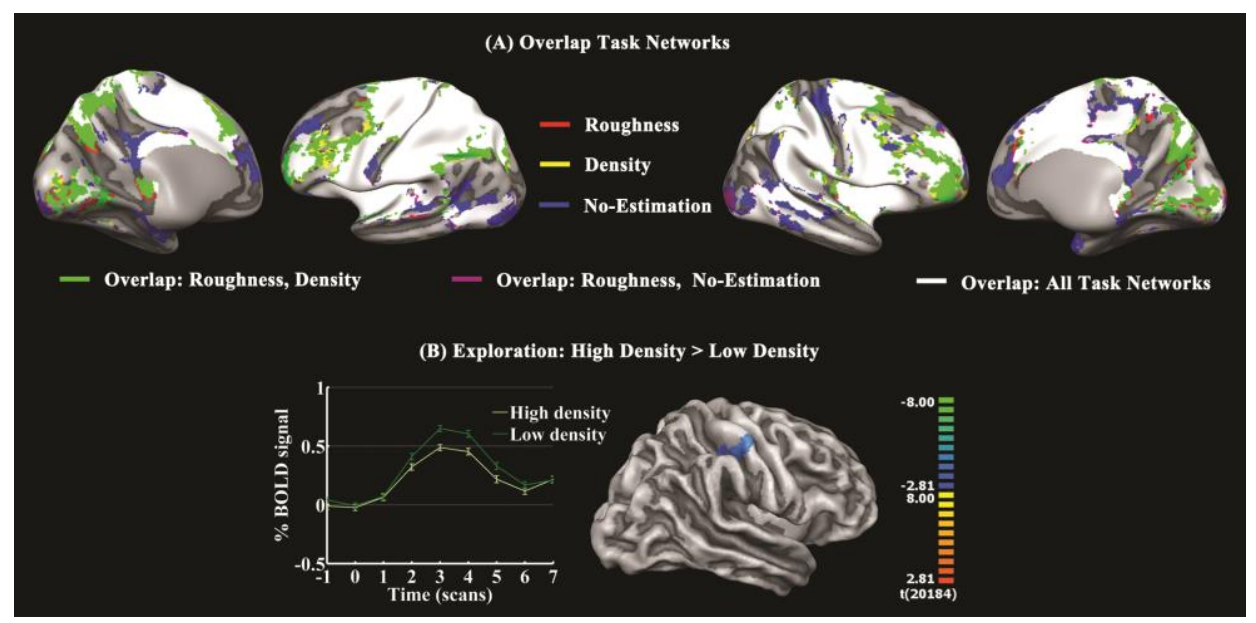

Figure 8. Results of univariate data analysis. (A) Overlap of the cortical networks involved in haptic texture exploration of all three tasks. (B) The effect of perceived density on a region in the ipsilateral PoCG; t-value [Talairach coordinate] of the peak voxel in that cluster: -4.43 [43, -27, 44].

\section{Multivariate data analysis}

Effects of Task Instructions. For the task classification haptic exploration trials were labeled according to the task instructions, i.e. roughness versus spatial density estimation, irrespective of the perceived texture properties. The classifier performance of distinguishing fMRI patterns elicited by haptic texture exploration between the two tasks was significant at the grouplevel for both early sensory and higher-order brain regions. The mean accuracy across all eight subjects reached 56.5\% in the PoCG (chance level: 51.6\%, p = 0.004). This was significantly above the empirical chance level as determined by the exact permutation test. Similar results were obtained in all other regions of interest. Both the mean classification accuracy of $57.9 \%$ (chance level: $51.3 \%, \mathrm{p}=0.004$ ) in the operculo-insular region and of $58.7 \%$ (chance level: $51.5 \%, \mathrm{p}=0.004)$ in the posterior parietal cortex were significant at the group level. Significant above chance classification was also confirmed for the lateral prefrontal cortex $(58.9 \%$, chance level: $51.7 \%, \mathrm{p}=0.008)$, the posterior occipital cortex $(56.1 \%$, chance level: $51.6 \%, \mathrm{p}=0.012)$ and the ventral temporal cortex $(56.2 \%$, chance level: $51.4 \%, \mathrm{p}=0.024)$.

Voxels in the PoCG mask that were discriminative for task classification and were consistent across at least 6 of 8 subjects over at least $25 \%$ RFE selection levels were observed at a typical location for tactile stimulation of the fingers, just posterior to the hand knob area of the contralateral motor cortex (Yousry et al., 1997; Stippich et al., 1999). However, discriminative voxels were not restricted to that region, but extended to more 
inferior and superior sites of the PoCG. Moreover, even the ipsilateral PoCG showed a contribution to task classification, albeit less pronounced than its contralateral match. The group discrimination map of the PoCG overlapped with BA $3 \mathrm{~b}$ and BA 1 . In addition to these rather early somatosensory regions, voxels in the posterior parietal cortex mask also survived the thresholding criterion for the group discrimination map, i.e. in the bilateral supramarginal gyrus, the superior parietal lobule and the anterior and posterior operculoinsular cortex. The parietal opercular regions covered the somatosensory fields $\mathrm{OP} 1 / 4$ and OP3. In the visual cortex discriminative voxels were revealed in the posterior calcarine sulcus (pCS) bordering the cuneus and the lateral pOCC and in higher-order regions of the ventral temporal cortex, i.e. primarily in the anterior collateral sulcus $(\mathrm{aCoS})$, but also in the fusiform gyrus (FG), the parahippocampal gyrus (PhG) and the occipitotemporal sulcus (OtS). Finally, voxels discriminating task instructions were also found in the posterior part of the bilateral superior frontal sulcus close to the frontal eye fields (FEF) and in a left-hemispheric middle frontal gyrus (MFG) region.

Effects of Perceived Density. Haptic texture exploration trials within the spatial density judgment task were labeled according to individual density ratings. This resulted in two trial categories that represented textures that were perceived as either dense or sparse. The classifier performance for density trials exceeded the empirical chance level in three of the six pre-defined cortical areas, with two of them representing early sensory regions. One of them was the PoCG that showed an average classification performance of $59.1 \%$ across subjects (chance level: $52.1 \%, \mathrm{p}=0.031$ ) and the other region was the pOCC that reached a similar above chance accuracy of $57.3 \%$ (chance level: $52.5 \%, \mathrm{p}$ $=0.028)$. The third cortical site that revealed a significant classifier performance across subjects was the PPC with 59.2\% (chance level: $52.1 \%, \mathrm{p}=0.012$ ).

The most discriminative voxels for perceived spatial density classification within the PoCG were located at similar sites as for task classification, including the region opposite to the hand knob area of the motor cortex in both hemispheres. The ipsilateral cluster overlapped with the effect of perceived density identified in the univariate data anlysis. There was an additional cluster in the superior PoCG of the contralateral hemisphere and one in the inferior PoCG of the ipsilateral cortex. The group discrimination map in the PoCG overlapped with Brodmann areas $3 \mathrm{~b}$ and 1, similar to the task discrimination. Voxels that were consistent across at least 6 of 8 subjects over at least $25 \%$ RFE selection levels were furthermore located in the bilateral posterior calcarine sulcus extending to the lateral portion of the pOCC. The spatial properties of the group discrimination map in the PoCG and the pOCC resemble the results of the task classification within the same brain regions. However, in contrast to the roughness and density task classification, the discrimination map of trial classification according to perceived spatial density only showed discriminative voxels consistent across subjects in the left PPC, 
contralateral to the exploring hand. These voxels were restricted to the superior parietal lobule and the angular gyrus.

Effects of Perceived Roughness. For the roughness judgment task haptic exploration trials were labeled according to the individual trial ratings during the fMRI scanning session. We used the trials in the high and low roughness category for classification of the fMRI patterns. Significant above chance classification of perceived roughness was only found for two brain regions, i.e. the operculo-insular cortex and the ventral temporal cortex with a mean group accuracy of $57.8 \%$ (chance level: $52.2 \%, \mathrm{p}=0.020$ ) and $56.3 \%$ (chance level: $52.6 \%, \mathrm{p}=0.031$ ) respectively. None of the other predefined brain areas showed classification performances that were significant across subjects.

Discriminative voxels for roughness classification across subjects were observed bilaterally in posterior and anterior parts of the operculo-insular cortex, not restricted to a specific location, but covering the somatoesensory parietal opercular regions OP1 and OP3. This is in contrast to the group discrimination map within the ventral temporal cortex; here the most discriminative voxels are clearly clustered in the collateral sulcus and middle fusiform gyrus of both hemispheres and in the right occipito-temporal sulcus. Although not completely identical there is some spatial resemblance of the discrimination map for task classification and perceived roughness classification in the ventral temporal cortex.

The results of the task, roughness and spatial density classification are summarized in Figure 9.

Effects of Stimulus Spacing. Trial labeling according to stimulus spacing was performed for the haptic exploration intervals in the No-Estimation runs. fMRI patterns were labeled as close spacing when subjects explored textures with inter-dot distances of $1.50 \mathrm{~mm}$ and $2.25 \mathrm{~mm}$. Trials were grouped to the wide spacing category when subjects touched textures with inter-dot spacings of $5.50 \mathrm{~mm}$ and $8.25 \mathrm{~mm}$. None of the predefined regions showed average classification accuracies for texture spacing that exceeded the empirical chance level. The average classifier performance across subjects ranged from $51.5 \%$ in the posterior parietal cortex to $54.8 \%$ in the postcentral gyrus, while the empirical chance level ranged from $51.8 \%$ in the posterior occipital cortex to $52.5 \%$ in the posterior parietal cortex (see Figure 10). 


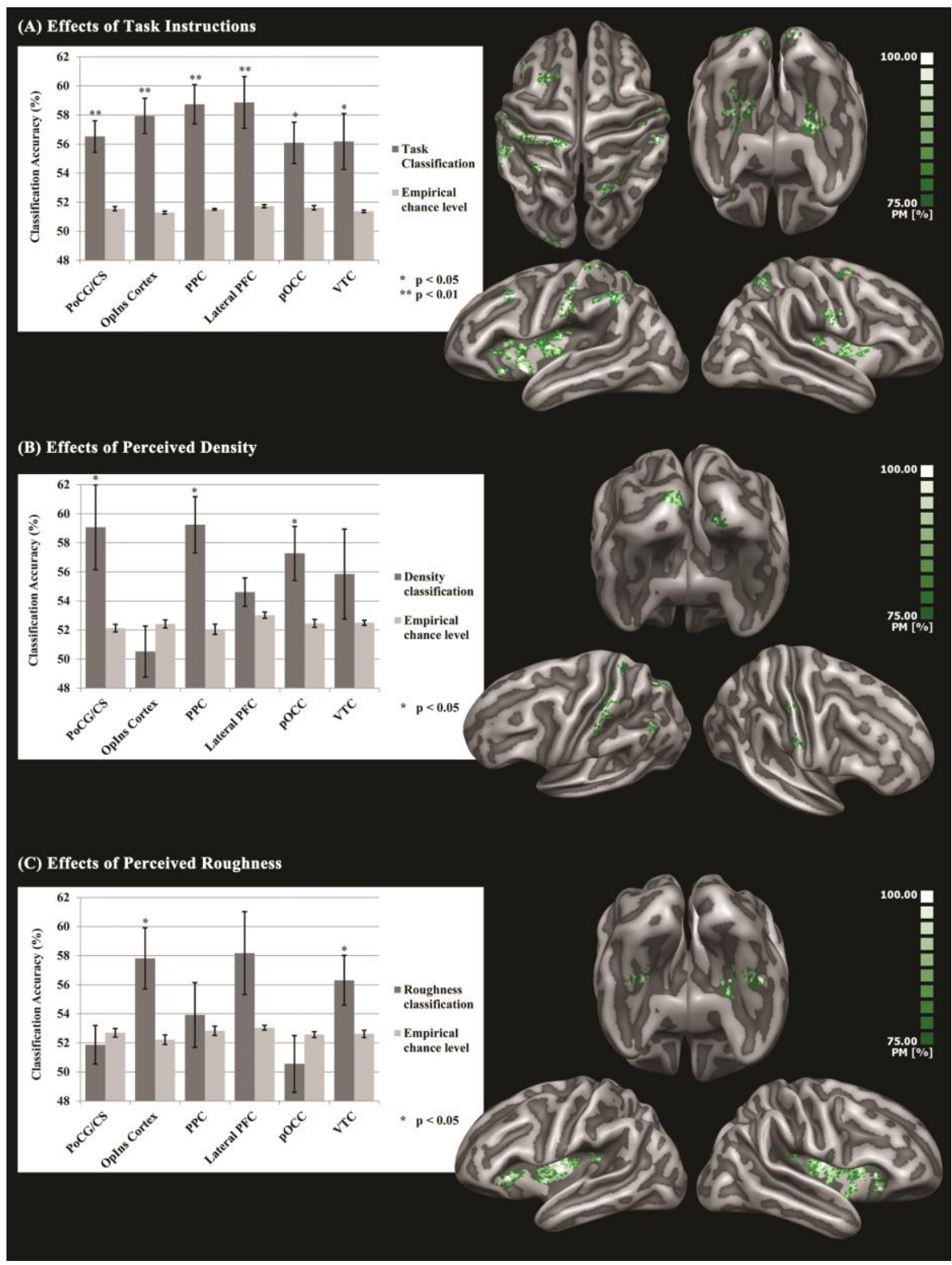

Figure 9. Results of multivariate data analysis. The left side of the figure shows the average classification accuracies across all subjects together with the average permutation accuracies for all predefined regions; the bars represent the standard error of the mean (SEM). In the right panel group discrimination maps are presented on the average cortex reconstruction of all subjects. The color-coding of the vertices represents the consistency across subjects within the different predefined regions. Green-colored voxels outlasted at least $25 \%$ of the RFE selection steps in at least 6 out of 8 subjects (75\%), while white-colored voxels survived in all subjects (100\%). 


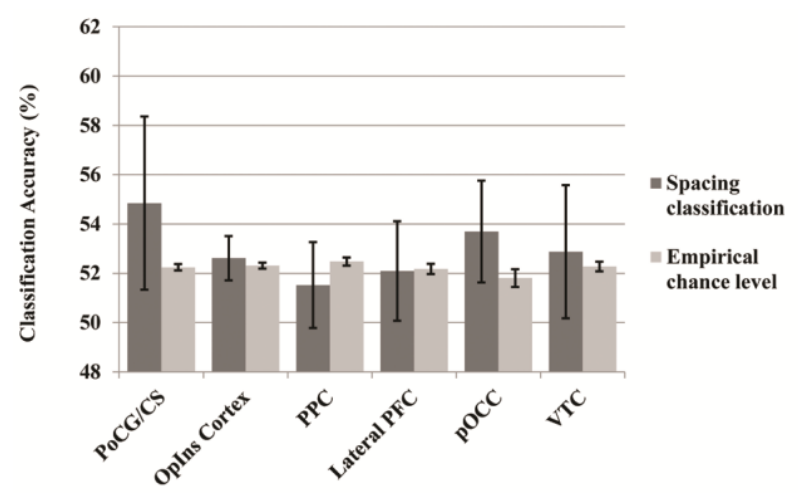

Figure 10. Results of spacing classification in the No-Estimation runs. Average classification accuracies across all subjects together with the average permutation accuracies for all predefined regions; the bars represent the standard error of the mean (SEM).

\section{DISCUSSION}

In the current study we combined psychophysical and imaging methods to compare fMRI signal patterns elicited by the haptic exploration of different dot pattern textures in a roughness and spatial density estimation task. We investigated which of the previously suggested brain regions convey information indicative of the performed haptic task, irrespective of the tactile stimulus space. In addition, we asked whether the same regions also hold information about the level of perceived texture characteristics, i.e. roughness and spatial density. We assume that perceived spatial density represents a one to one mapping between the physical and the perceptual space and therefore hypothesized an involvement of primarily early sensory cortices. In contrast, perceived roughness likely requires integration of multiple factors; hence we hypothesized an involvement of higher-order association cortices.

Overall, the results of the behavioral data analysis corroborate earlier psychophysical findings with a simple linear relationship of inter-dot spacing and perceived spatial density and an inverted U-shape function of perceived roughness (Connor et al., 1990; Merabet et al., 2004; Gescheider et al., 2005; Eck et al., 2013a; Eck et al., 2013b; Gescheider and Wright 2013). The negative linear relationship between the inter-dot spacing of dot pattern textures and perceived spatial density was consistent across subjects. The psychophysical curve of perceived roughness however showed a stronger variation across subjects. Although roughness ratings for all participants consistently increased up to an inter-dot spacing of 3.50 or $4.50 \mathrm{~mm}$, the slope of the subsequent decrease was affected by inter-individual variability. As already mentioned before this higher inter-individual variability in perceived roughness ratings could be an effect of the minimally sampled stimulus space in the current study. 
Alternatively, subjects might have used different rating strategies, in particular for wide dot spacing textures. Since the dot-occurrences were rarer for wider inter-dot spacings, some subjects might have switched from judging the roughness of the whole pattern (the macrostructure of the pattern) to judging the roughness of the single elements (the microstructure), i.e. the individual raised dots. This variation in rating strategies would explain the very shallow decrease of perceived roughness for wide dot patterns in some subjects. However, only 4 out of 12 participants showed this almost plateau-like pattern for textures with an inter-dot spacing of $4.5 \mathrm{~mm}$ and above. In order to reduce variability in rating strategies and to assure an unambiguous roughness categorization of the textures, eight participants were included in the fMRI data analysis.

In the fMRI data analysis we focused on the haptic exploration of the textures in all three conditions, i.e. roughness and density rating as well as haptic exploration without any cognitive task demands. Dot pattern exploration elicited activation in a very similar cortical network across all three conditions, involving regions necessary for task execution. Especially the lateral prefrontal part of the network was more extended in the rating tasks, which might represent the additional requirement of comparing the stimuli to the taskrelevant internal standard and linking this to an appropriate (future) response. This is consistent with the essential role of the lateral prefrontal cortex in higher-order control of cognitive processes and behavior (see Petrides (2005); Tanji and Hoshi (2008) for reviews on the function of the lateral PFC). In the two rating tasks subjects had to maintain the encoded haptic information until the rating interval of the trial started $9-13 \mathrm{sec}$ after trial onset. Hence, the considerable working memory encoding requirements might have added to the more extensive involvement of the prefrontal cortex (Kaas et al., 2013).

The univariate analysis of the neuroimaging data revealed a significant effect of perceived spatial density in the right but not in the left postcentral cortex. This result is unexpected considering that subjects performed the task with their right hand. However, an event-related averaging analysis in that region clearly showed that this effect was not due to signal differences below baseline level. Furthermore, although not as common in the literature as contralateral activation changes, effects in ipsilateral postcentral regions have been reported before for tactile tasks, as for example by Debowska et al. (2013); van Ede et al. (2013) and Sathian et al. (2013). Interestingly, there was neither an effect of perceived roughness nor of task instructions (roughness versus density estimation) in the univariate data analysis. This lack of significant findings is quite surprising in the light of previous studies showing effects of perceived roughness in the insular and prefrontal cortex (Kitada et al., 2005; Simões-Franklin et al., 2011) and effects of task instructions in the intraparietal sulcus and postcentral regions (Merabet et al., 2007). A reduced power in our study resulting from a relatively small sample size of $\mathrm{N}=8$ and the short stimulus exploration of $4 \mathrm{sec}$, which was considerably shorter than in the above mentioned experiments, might explain the discrepancies in the findings. 
In contrast to the univariate approach, the multivariate analysis of the same data revealed effects of task instructions, perceived spatial density and roughness, indicating that multivariate techniques are indeed more sensitive to pick up subtle difference in the activation patterns of haptic tasks. All of the predefined regions showed significant above chance classification of the estimation tasks across subjects. This suggests that not only higher-order regions such as the lateral PFC, the OpIns cortex, the PPC and the VTC convey information indicative of the performed haptic task, but that this information is partly represented in early sensory regions, i.e. the PoCG/CS and the pOCC. The effect of task in the somatosensory cortex was not restricted to but included the cortical input regions for cutaneous information (Kaas et al., 1993), i.e. BA $3 \mathrm{~b}$ constituting the primary somatosensory cortex (SI) and BA 1 as a projection site of BA 3b. Notably, there was no difference in the employed texture set and the experimental design of both rating tasks; the only difference was the task instruction for the subjects. Furthermore, the involvement of OP1/4 and OP3 in task classification supports earlier findings indicating an involvement of these somatosensory parietal opercular fields in texture perception (e.g., Sathian et al., 2011; Stilla and Sathian, 2008). Interestingly, significant classification effects were not restricted to somatosensory regions and the prefrontal cortex, which would be expected from a purely tactile task. Classical visual regions in the posterior occipital cortex and the ventral temporal cortex showed a similar pattern for task classification. This is consistent with the body of haptic literature in recent years showing an involvement of visual cortex regions in tactile tasks. First, a visual cortex contribution was reported for a macrospatial task, i.e. grating orientation discrimination (Sathian et al., 1997). Thereafter this finding was extended to many other macro- but also microspatial tasks (Amedi et al., 2001; Stoesz et al., 2003; Merabet et al., 2007; Peltier et al., 2007; Deshpande et al., 2008; Sathian et al., 2011). Based on the task classification results we cannot infer which of these regions are involved in spatial density estimation of the dot pattern textures and which contribute to roughness perception. To that end we tested whether some of these regions also hold information that discriminates texture trials that were perceived by the participants as either rough/dense or smooth/sparse. In agreement with earlier studies we found significant above chance classification of the spatial density classes in early somatosensory (BA 3b and BA 1) and visual cortex (Zangaladze et al., 1999; Merabet et al., 2004; Merabet et al., 2007), but also in two higher parietal regions, namely the left superior parietal lobule and the angular gyrus. Interestingly though, SVM classification of physical wide and close inter-dot spacing did not exceed the empirical chance level in the same regions. At first sight this is somewhat surprising considering that the texture categorization according to physical inter-dot spacing was identical to categorization according to perceived spatial density, as indicated by the negative linear relationship of the physical and perceptual texture characteristics, observed in the behavioral results. Hence, perceived spatial density indeed appears to be a one to one 
mapping of the physical stimulus space. However, as postulated in the introduction the enhanced attention to the physical stimulus space, induced by the spatial density estimation instruction, might have promoted the cortical processing of small differences in the tactile texture set. Such top-down influences on tactile stimulus processing have been observed as early as in the primary somatosensory cortex (Sterr et al., 2007; van Ede et al., 2013) and are also well known for visual processing (Treue 2001; Andersen et al., 2008). Furthermore, the involvement of the posterior occipital cortex in a mere tactile task is in agreement with the idea that primary sensory regions share similar representations, an idea suggested by Merabet et al. (2004) and further elaborated by Eck et al. (2013a). In summary, the results of the perceived spatial density and physical spacing SVM classification indicate that differences in the physical stimulus space of tactile dot pattern textures are represented in early sensory regions of the visual and the somatosensory cortex as well as in higher parietal areas, but that attention to these differences is necessary to reveal this information. Nevertheless, in our previous study we showed small but significant parametric effects of stimulus spacing in the pOCC, also in a task without any cognitive evaluation demands (Eck et al., 2013a). Therefore, it is possible that similar above chance classifications could have been reached for wide and close spacing categories with a more powerful design, e.g. more stimulus repetitions as was the case in our previous study.

In contrast to perceived spatial density, perceived roughness information appears to be conveyed predominantly by higher-order cortical areas as indicated by classifications above the empirical chance level in the operculo-insular cortex and the ventral temporal cortex. The parietal opercular regions included OP1, the homologue of SII (Eickhoff et al., 2006) and OP3, both of which have been implicated in roughness perception before (Burton et al., 2008; Simões-Franklin et al., 2011). The results of the current study also corroborate earlier reports of an involvement of the insula in tactile roughness perception (Kitada et al., 2005). The significant findings in higher-order regions could be interpreted as support of our hypothesis that perceived roughness cannot be solely explained by a simple representation of the physical stimulus space which is coded in the primary sensory cortex, but is rather an integration of multiple factors likely to be represented in association cortex. However, the lack of significant findings in early sensory cortices could also point to an insufficient sensitivity of our experimental design to reveal roughness-related signal changes in these regions, as it was previously shown that neuronal activity in monkey primary somatosensory cortex codes for texture differences that represented differences in perceived roughness (Sinclair and Burton 1991; Jiang et al., 1997). However, these experiments employed stimuli in which either the groove width of linear gratings or the inter-dot spacing of dot patterns fell in a range where they are expected to be approximately linearly related to perceived roughness (Meftah et al., 2000; Lawrence et al., 2007). Hence, the confounded spatial and roughness characteristics of the stimuli renders it almost 
impossible to answer the question which texture property is eventually coded by the neuronal cells in SI. Nevertheless, this does not exclude the possibility that roughness differences are indeed represented in the primary sensory cortex, but that our experimental design was not sensitive enough to pick up these differences.

In addition to the significant classification of perceived roughness categories in the operculo-insular cortex, the same was found for the ventral temporal cortex. The most discriminative voxels in the collateral sulcus for high and low roughness classification were close to fMRI activation peaks previously reported for visual attention to surface textures (Cant and Goodale 2007; Cant and Xu 2012). This would be expected as the VTC region in our study was defined based on a visual texture localizer. Interestingly though, the classification results show that this region also conveys information indicative for tactile texture roughness. Although, no fMRI signal differences between fine and coarse tactile textures were reported for the occipital and temporal cortex in a study by Simões-Franklin et al. (2011), several clusters in the same cortices showed a general contribution to a roughness categorization task. The results of a visual material study by Hiramatsu et al. (2011) also point to a contribution of higher-order visual regions to roughness or texture perception in general. The authors showed that activity in the FG/CoS reflects dissimilarity ratings of materials as assessed by different tactile adjective pairs (e.g. smooth — rough). To our knowledge, the present study is the first revealing signal changes in the anterior medial ventral visual cortex specific for tactile roughness. This result extends the findings of a vast body of literature providing strong evidence for a visual cortex involvement in several micro- and macrospatial tactile tasks such as shape perception (Amedi et al., 2001; Miquée et al., 2008), orientation discrimination (Sathian et al., 1997; Zangaladze et al., 1999), threshold-tracking and spacing judgments (Merabet et al., 2004; Merabet et al., 2007). Analogous to the primary somatosensory and visual cortex sharing a representation of spatial density, the operculo-insular and ventral temporal cortex could share a representation for perceived roughness.

We cannot be certain whether our results can be generalized to other kinds of textures. Sutu et al. (2013) reported for example a linear increase in perceived roughness with increasing inter-dot spacing, using very similar dot patterns but with slightly higher dots. The authors suggested that a simple intensive code (mean discharge rate of primary mechanoreceptive afferents) may explain these roughness-related changes. Furthermore, they argued that this interpretation is supported by the finding that neuronal discharge rates in monkey SI increase over a similar range as roughness perception did in their experiment (Jiang et al., 1997). Although this is an interesting conjecture, to our knowledge no neuroimaging study has been published so far reporting roughness related changes in the human primary somatosensory cortex. In contrast to the higher dot pattern textures, Sutu and colleagues (Sutu et al., 2013) found a comparable inverted U-shape function as we did when testing 
roughness perception on dot pattern stimuli with lower dots. According to Sutu et al. (Sutu et al., 2013), their findings indicate that subjects judged texture roughness for the ascending limb of the inverted $\mathrm{U}$ function but some other texture quality for the descending limb. If this interpretation were true, it would render this stimulus set useless for investigating roughness-related changes in the human brain. However, the consistency of roughness-related activation changes in the human operculo-insular cortex in our and other studies using different textures, i.e. sandpaper and linear gratings (Kitada et al., 2005; Simões-Franklin et al., 2011), leads us to believe that our subjects indeed judged roughness. This consistency with other studies using different texture sets corroborates our findings of roughness-related changes in the operculoinsular cortex and the ventral temporal cortex.

\section{CONCLUSION}

In the current study we revisited the question of the neural representation of perceiving different tactile texture properties. Based on previous studies we hypothesized that perceived spatial density of tactile dot pattern textures is primarily represented in early sensory cortex, as spatial density represents a one to one mapping of the perceptual and the physical stimulus space, whereas perceived roughness is rather represented in higherorder cortical structures, as an integration of multiple factors is necessary.

The psychophysical and neuroimaging results are coherent in their implications. Both early as well as higher-order visual, somatosensory and prefrontal regions convey information indicative of the tactile task performed on an identical set of textures, i.e. judging spatial density versus roughness. However, while different levels of perceived spatial density can be decoded already from fMRI activation patterns in early sensory but also in higher somatosensory association cortices, perceived roughness is solely detected in higher-order regions of the ventral visual cortex and the operculo-insular cortex. These cortical differences seem to corroborate the psychophysical findings implicating that perceived spatial density represents a simple mapping of the physical stimulus space, via a negative linear function of inter-dot spacing of the tactile dot patterns. Perceived roughness, on the other hand, cannot be described by such a simple linear function, but an inverted $U$-shape function provides an adequate description of the changes in perceived roughness with varying inter-dot distances of this stimulus set. These results confirm and extend earlier reports by showing that the visual cortex is not only involved in judging spatial characteristics of tactile textures, but also contributes to tactile roughness perception. A possible interpretation of the findings includes a shared representation of perceived texture characteristics in somatosensory and visual cortices, with spatial density relying on early sensory regions and roughness on higher association cortices. 


\section{ACKNOWLEDGMENTS}

This work was supported by the European Community's Seventh Framework Programme FP7/2007-2013 (PITN-GA-2008-214728) and the BrainGain Smart Mix Programme of the Netherlands Ministry of Economic Affairs and the Netherlands Ministry of Education, Culture and Science (SSM06011). We would like to thank Alan Meeson and Giancarlo Valente for their very helpful remarks on the data analysis and Valerie Goffaux for her great help with designing the visual texture localizer. 


\section{REFERENCES}

Amedi, A., Malach, R., Hendler, T., Peled, S., Zohary, E., 2001. Visuo-haptic objectrelated activation in the ventral visual pathway. Nat. Neurosci. 4, 324-330.

Andersen, S.K., Hillyard, S.A., Müller, M.M., 2008. Attention facilitates multiple stimulus features in parallel in human visual cortex. Curr. Biol. 18, 1006-1009.

Bergmann Tiest, W.M., Kappers, A.M.L., 2006. Analysis of haptic perception of materials by multidimensional scaling and physical measurements of roughness and compressibility. Acta Psychol. (Amst.) 121, 1-20.

Burton, H., Sinclair, R.J., Wingert, J.R., Dierker, D.L., 2008. Multiple parietal operculum subdivisions in humans: Tactile activation maps. Somatosens. Mot. Res. 25, 149-162.

Cant, J.S., Goodale, M.A., 2007. Attention to form or surface properties modulates different regions of human occipitotemporal cortex. Cereb. Cortex 17, 713731.

Cant, J.S., Xu, Y., 2012. Object ensemble processing in human anterior-medial ventral visual cortex. J. Neurosci. 32, 7685-7700.

Connor, C.E., Hsiao, S.S., Phillips, J.R., Johnson, K.O., 1990. Tactile roughness: neural codes that account for psychophysical magnitude estimates. J. Neurosci. 10, 3823-3836.

Culham, J.C., Valyear, K.F., 2006. Human parietal cortex in action. Curr. Opin. Neurobiol. 16, 205-212.

De Martino, F., Valente, G., Staeren, N., Ashburner, J., Goebel, R., Formisano, E., 2008. Combining multivariate voxel selection and support vector machines for mapping and classification of fMRI spatial patterns. Neuroimage 43, 44-58.

Debowska, W., Wolak, T., Soluch, P., Orzechowski, M., Kossut, M., 2013. Design and evaluation of an innovative MRI-compatible Braille stimulator with high spatial and temporal resolution. J. Neurosci. Methods 213, 32-38.

Deshpande, G., Hu, X., Stilla, R., Sathian, K., 2008. Effective connectivity during haptic perception: A study using Granger causality analysis of functional magnetic resonance imaging data. Neuroimage 40, 1807-1814.

Eck, J., Kaas, A.L., Goebel, R., 2013a. Crossmodal interactions of haptic and visual texture information in early sensory cortex. Neuroimage 75, 123 - 135.

Eck, J., Kaas, A.L., Mulders, J.L., Goebel, R., 2013b. Roughness perception of unfamiliar dot pattern textures. Acta Psychol. (Amst.) 143, 20-34.

Eickhoff, SB, Amunts K, Mohlberg H, Zilles K. 2006. The human parietal operculum. II. Stereotaxic maps and correlation with functional imaging results. Cereb. Cortex. 16, 268-279.

Eickhoff, S.B., Grefkes, C., Fink, G.R., Zilles, K., 2008. Functional lateralization of face, hand, and trunk representation in anatomically defined human somatosensory areas. Cereb. Cortex 18, 2820-2830.

Eickhoff, S.B., Grefkes, C., Zilles, K., Fink, G.R., 2007. The somatotopic organization of cytoarchitectonic areas on the human parietal operculum. Cereb. Cortex 17, 1800-1811.

Forman, S.D., Cohen, J.D., Fitzgerald, M., Eddy, W.F., Mintun, M.A., Noll, D.C., 1995. Improved assessment of significant activation in functional magnetic resonance imaging (fMRI): use of a cluster-size threshold. Magn. Reson. Med. 33, 636647. 
Frost, M.A., Goebel, R., 2012. Measuring structural-functional correspondence: spatial variability of specialised brain regions after macro-anatomical alignment. Neuroimage 59, 1369-1381.

Gescheider, G.A., Bolanowski, S.J., Greenfield, T.C., Brunette, K.E., 2005. Perception of the tactile texture of raised-dot patterns: A multidimensional analysis. Somatosens. Mot. Res. 22, 127-140.

Gescheider, G.A., Wright, J.H., 2013. Roughness perception in tactile channels: Evidence for an opponent process in the sense of touch. Somatosens. Mot. Res. 30, 120-132.

Goebel, R., Esposito, F., Formisano, E., 2006. Analysis of functional image analysis contest (FIAC) data with Brainvoyager QX: From single-subject to cortically aligned group general linear model analysis and self-organizing group independent component analysis. Hum. Brain Mapp. 27, 392-401.

Goulas, A., Uylings, H.B.M., Stiers, P., 2012. Unravelling the intrinsic functional organization of the human lateral frontal cortex: A parcellation scheme based on resting state fMRI. J. Neurosci. 32, 10238-10252.

Hiramatsu, C., Goda, N., Komatsu, H., 2011. Transformation from image-based to perceptual representation of materials along the human ventral visual pathway. Neuroimage 57, 482-94.

Hollins, M., Bensmaia, S., Karlof, K., Young, F., 2000. Individual differences in perceptual space for tactile textures: Evidence from multidimensional scaling. Percept. Psychophys. 62, 1534-1544.

Hollins, M., Faldowski, R., Rao, S., Young, F., 1993. Perceptual dimensions of tactile surface texture: A multidimensional scaling analysis. Percept. Psychophys. 54, 697-705.

Jiang, W., Tremblay, F., Chapman, C.E., 1997. Neuronal encoding of texture changes in the primary and the secondary somatosensory cortical areas of monkeys during passive texture discrimination. J. Neurophysiol. 77, 1656-1662.

Kaas, J.H., 1993. The functional organization of somatosensory cortex in primates. Ann. Anat. 175, 509-518.

Kaas, A.L., van Mier, H., Visser, M., Goebel, R., 2013. The neural substrate for working memory of tactile surface texture. Hum. Brain Mapp. 34, 1148-1162.

Kitada, R., Hashimoto, T., Kochiyama, T., Kito, T., Okada, T., Matsumura, M., Lederman, S.J., Sadato, N., 2005. Tactile estimation of the roughness of gratings yields a graded response in the human brain: an fMRI study. Neuroimage 25, 90-100.

Lawrence, M.A., Kitada, R., Klatzky, R.L., Lederman, S.J., 2007. Haptic roughness perception of linear gratings via bare finger or rigid probe. Perception 36, 547557.

Ledberg, A., O'Sullivan, B.T., Kinomura, S., Roland, P.E., 1995. Somatosensory activations of the parietal operculum of man. A PET study. Eur. J. Neurosci. 7, 1934-1941.

Lederman, S.J., Thorne, G., Jones, B., 1986. Perception of texture by vision and touch: Multidimensionality and intersensory integration. J. Exp. Psychol. Hum. Percept. Perform. 12, 169-180.

Mazzola, L., Faillenot, I., Barral, F.-G., Mauguiere, F., Peyron, R., 2012. Spatial segregation of somato-sensory and pain activations in the human operculoinsular cortex. Neuroimage 60, 409-418. 
Meftah, E.-M., Belingard, L., Chapman, C.E., 2000. Relative effects of the spatial and temporal characteristics of scanned surfaces on human perception of tactile roughness using passive touch. Exp. Brain Res. 132, 351-361.

Merabet, L.B., Swisher, J.D., McMains, S.A., Halko, M.A., Amedi, A., Pascual-Leone, A., Somers, D.C., 2007. Combined activation and deactivation of visual cortex during tactile sensory processing. J. Neurophysiol. 97, 1633-1641.

Merabet, L., Thut, G., Murray, B., Andrews, J., Hsiao, S., Pascual-Leone, A., 2004. Feeling by sight or seeing by touch? Neuron 42, 173-179.

Miquée, A., Xerri, C., Rainville, C., Anton, J.-L., Nazarian, B., Roth, M., ZennouAzogui, Y., 2008. Neuronal substrates of haptic shape encoding and matching: A functional magnetic resonance imaging study. Neuroscience 152, 29-39.

O'Sullivan, B.T., Roland, P.E., Kawashima, R., 1994. A PET study of somatosensory discrimination in man. microgeometry versus macrogeometry. Eur. J. Neurosci. 6, 137-148.

Peltier, S., Stilla, R., Mariola, E., LaConte, S., Hu, X., Sathian, K., 2007. Activity and effective connectivity of parietal and occipital cortical regions during haptic shape perception. Neuropsychologia 45, 476-483.

Petrides, M., 2005. Lateral prefrontal cortex: architectonic and functional organization. Philos. Trans. R. Soc. Lond., B, Biol. Sci. 360, 781-795.

Picard, D., Dacremont, C., Valentin, D., Giboreau, A., 2003. Perceptual dimensions of tactile textures. Acta Psychol. (Amst.) 114, 165-184.

Roland, P.E., O'Sullivan, B., Kawashima, R., 1998. Shape and roughness activate different somatosensory areas in the human brain. Proc. Natl. Acad. Sci. U.S.A. 95, 3295-3300.

Sanchez-Panchuelo, R.M., Francis, S., Bowtell, R., Schluppeck, D., 2010. Mapping human somatosensory cortex in individual subjects with 7T functional MRI. J. Neurophysiol. 103, 2544-2556.

Sathian, K., Deshpande, G., Stilla, R., 2013. Neural changes with tactile learning reflect decision-level reweighting of perceptual readout. J. Neurosci. 33, 5387-5398.

Sathian, K., Lacey, S., Stilla, R., Gibson, G.O., Deshpande, G., Hu, X., LaConte, S., Glielmi, C., 2011. Dual pathways for haptic and visual perception of spatial and texture information. Neuroimage 57, 462-475.

Sathian, K., Zangaladze, A., Hoffman, J.M., Grafton, S.T., 1997. Feeling with the mind's eye. Neuroreport 8, 3877-3881.

Simões-Franklin, C., Whitaker, T.A., Newell, F.N., 2011. Active and passive touch differentially activate somatosensory cortex in texture perception. Hum. Brain Mapp. 32, 1067-1080.

Sinclair, R.J., Burton, H., 1991. Neuronal activity in the primary somatosensory cortex in monkeys (Macaca mulatta) during active touch of textured surface gratings: responses to groove width, applied force, and velocity of motion. J. Neurophysiol. 66, 153-169.

Sterr, A., Shen, S., Zaman, A., Roberts, N., Szameitat, A., 2007. Activation of SI is modulated by attention: a random effects fMRI study using mechanical stimuli. Neuroreport 18, 607-611.

Stilla, R., Sathian, K., 2008. Selective visuo-haptic processing of shape and texture. Hum. Brain Mapp.. 29, 1123-1138. 
Stippich, C., Hofmann, R., Kapfer, D., Hempel, E., Heiland, S., Jansen, O., Sartor, K., 1999. Somatotopic mapping of the human primary somatosensory cortex by fully automated tactile stimulation using functional magnetic resonance imaging. Neurosci. Lett. 277, 25-28.

Stoesz, M.R., Zhang, M., Weisser, V.D., Prather, S.C., Mao, H., Sathian, K., 2003. Neural networks active during tactile form perception: common and differential activity during macrospatial and microspatial tasks. Int. J. Psychophysiol. 50, 41-49.

Stringer, E.A., Chen, L.M., Friedman, R.M., Gatenby, C., Gore, J.C., 2011. Differentiation of somatosensory cortices by high-resolution fMRI at $7 \mathrm{~T}$. Neuroimage 54, 1012-1020.

Sutu, A., Meftah, E.-M., Chapman, C.E., 2013. Physical determinants of the shape of the psychophysical curve relating tactile roughness to raised-dot spacing: implications for neuronal coding of roughness. J. Neurophysiol. 109, 14031415.

Suykens, J.A.K., De Brabanter, J., Lukas, L., Vandewalle, J., 2002. Weighted least squares support vector machines: robustness and sparse approximation. Neurocomputing. 48, 85-105.

Tanji, J., Hoshi, E., 2008. Role of the lateral prefrontal cortex in executive behavioral control. Physiol. Rev. 88, 37-57.

Tremblay, F., Ageranioti-Bélanger, S.A., Chapman, C.E., 1996. Cortical mechanisms underlying tactile discrimination in the monkey. I. Role of primary somatosensory cortex in passive texture discrimination. J. Neurophysiol. 76, $3382-3403$.

Treue, S., 2001. Neural correlates of attention in primate visual cortex. Trends Neurosci. 24, 295-300.

van Ede, F., de Lange, F.P., Maris, E., 2013. Anticipation increases tactile stimulus processing in the ipsilateral primary somatosensory cortex. Cereb. Cortex. [Epub ahead of print]

Yousry, T.A., Schmid, U.D., Alkadhi, H., Schmidt, D., Peraud, A., Buettner, A., Winkler, P., 1997. Localization of the motor hand area to a knob on the precentral gyrus. A new landmark. Brain 120, 141-157.

Zangaladze, A., Epstein, C.M., Grafton, S.T., Sathian, K., 1999. Involvement of visual cortex in tactile discrimination of orientation. Nature 401, 587-590. 


\section{Chapter 5}

Summary and general discussion 
The goal of this thesis was to broaden our understanding of haptic texture perception and its interaction with visual information as well as to shed some light on the underlying cortical mechanisms related to these perceptual processes and the physical texture characteristics. In the following sections, I will summarize the main findings of Chapters 2 through 4 . Subsequently, I will discuss them in relation to the body of literature to date and suggest directions for future research in this field.

Imagine being asked to judge the roughness of different samples of sandpaper. Probably this would not be the first time that you feel and see these kinds of textures. Hence, your pre-existing experience with the sandpaper might influence your roughness estimations, e.g. via the knowledge that the roughness of sandpaper crucially depends on the grit size and that this is a texture property that you can perceive visually and by touch. Hence, our visual and our haptic experience are tightly linked through our experience with different materials and textures. In Chapter 2 I investigated whether the similarity of the visual and haptic modality with respect to roughness estimation, as was for example shown by Lederman and Abbott (1981), is influenced by the pre-existing visual and haptic experience with the stimulus material. For a set of unfamiliar dot pattern textures, we observed an effect of haptic familiarity with the stimulus material on visual roughness perception. However, this effect was only apparent in a bimodal experimental context, in which subjects knew that they would be asked to judge the stimuli not only separately using vision and touch, but also in a combined visual-haptic condition. The dominant view in the literature that the haptic modality exerts a stronger influence on roughness perception than the visual modality (Heller, 1989; Lederman et al., 1986) was supported by the unidirectionality of the sensory familiarity effect. Visual roughness judgments were biased towards haptic roughness estimates when textures were first experienced in a condition with haptic input, but not vice versa. The strong resemblance of haptic and visual-haptic roughness estimations, observed in the same study, is another indication that haptic roughness information receives a stronger weight especially in bimodal settings. Chapter 2 also provides the basis for comparing the results of behavioral and neuroimaging studies employing the same dot pattern textures, by showing no differences in perceived roughness of real and virtual visual textures.

In summary Chapter 2 corroborates earlier findings by showing that haptic information dominates visual information in bimodal roughness judgments. Moreover, it demonstrates that not only the physical texture characteristics but also the sensory context influence roughness perception, with haptic information biasing visual roughness perception only in settings that require the combination of both sources of information to a bimodal judgment.

In Chapter 3 the interaction of haptic and visual texture information was further investigated at the cortical level. This fMRI study focused on the effect of physical stimulus properties and sensory modality on the cortical processing of textures. The results indicated that matching relative to non-matching visual 
and haptic texture information elicits a stronger BOLD response in the early visual cortex. This result is in line with an earlier reported overlap of the visual and haptic texture processing stream in a region of the posterior occipital cortex (Sathian et al., 2011; Stilla and Sathian, 2008). Interestingly, unimodal visual parametric texture variation had no effect on the BOLD response. In the haptic condition, however, a small effect of parametric texture variation was observed in the posterior occipital cortex. With matching visual-haptic texture information this parametric effect on the BOLD response was more extended in the visual cortex and reached significance in the postcentral gyrus and inferior parietal lobule. Overall these results challenge the view that visual and haptic texture information are processed independently in the brain. Much on the contrary, the present results suggest an interaction of both processing pathways at the cortical level.

The fMRI study described in Chapter 4 extends the findings from Chapter 3, which focused on effects of physical stimulus variations on cortical activations, to effects of perceptual texture characteristics on cortical processing of somatosensory information. This study showed that while differences in the perceived spatial density of a specific texture set are already represented in early sensory cortices, information on differences of perceived tactile roughness is primarily available in higher-order cortical areas, namely the operculo-insular and the medial ventral visual cortex. This is, to our knowledge, the first study reporting tactile roughness-related activation changes within the visual cortex.

The following section is used to develop a working model for visual and haptic texture processing, which is meant to integrate the findings of the studies reported in this thesis with knowledge acquired in recent years. Most importantly, it is meant as a starting point to develop new research questions for future studies.

\section{INTEGRATION OF FINDINGS IN A TEXTURE PROCESSING MODEL}

The model that I will introduce in this section, is based on the model that was originally described by Dijkerman and Haan (2007), and in which somatosensory processes subserving perception are distinguished from actionrelated processes at the cortical level, akin to the well-known two-pathway model of visual processing. The idea of a comparable distinction for somatosensory processing was built on evidence from lesion, neurophysiological and neuroimaging studies. The authors proposed that both pathways start in the anterior parietal cortex (SI) and further include SII, while being subsequently separated depending on the function of the somatosensory process. The primary and secondary somatosensory cortices, shared by both pathways, are mainly responsible for simple feature processing and feature integration. The processing stream related to perception, recognition and memory further projects from SII to the insula and the PPC, whereas the action- 
related pathway terminates in the PPC. The general distinction of visual processing into dorsal occipitoparietal and ventral occipitotemporal processing streams (Goodale and Milner, 1992) is well accepted today, although details of this key framework in visual neuroscience are continuously adapted to the growing body of evidence in this field (e.g. Kravitz et al., 2011). However, a similar dichotomy for somatosensory information processing, as proposed by Dijkerman and Haan (2007), has only recently grown in appeal. Several studies indicated distinct cortical processing streams for somatosensory information related to identification or recognition, i.e. the 'what' pathway, and information related to spatial localization or action, i.e. the 'where' or 'how' pathway (de Santis et al., 2007; Reed et al., 2005; van Boven et al., 2005). It was also shown that cortical areas forming both pathways partly overlap between sensory modalities (Renier et al., 2009, Sathian et al., 2011), indicating this segregation as a general organization principle across sensory modalities.

Here, I will focus on the perception-related 'what' pathway and propose differences and commonalities in the perception and processing of single texture dimensions, i.e. spatial density and roughness. First insights on the interaction of visual and somatosensory texture information at the cortical level are outlined as well. I propose a further distinction of the 'what' pathway into distinct but interacting cortical pathways for the processing of spatial density and roughness. Both pathways share the same sensory input regions for visual and tactile texture information, namely the primary visual and somatosensory cortex. However, while perceptual differences in spatial density are already represented in these early sensory cortices and are relayed to a dorsal pathway including the posterior parietal cortex, differences in perceived roughness are processed in a ventral pathway and are primarily represented in higher-order cortices, namely the operculo-insular cortex including SII and the ventral temporal cortex (see Figure 1). Hence, the suggested distinction in this model is two-fold. First it is assumed that spatial density is processed in a dorsal pathway, while roughness is processed in a ventral pathway. Second a hierarchical difference in the processing is assumed, with spatial density being represented at early cortical processing stages and roughness primarily at a higher processing stage. It is beyond question that information processing in both pathways is tightly linked; however, how the crosstalk takes place needs to be addressed in future studies. 


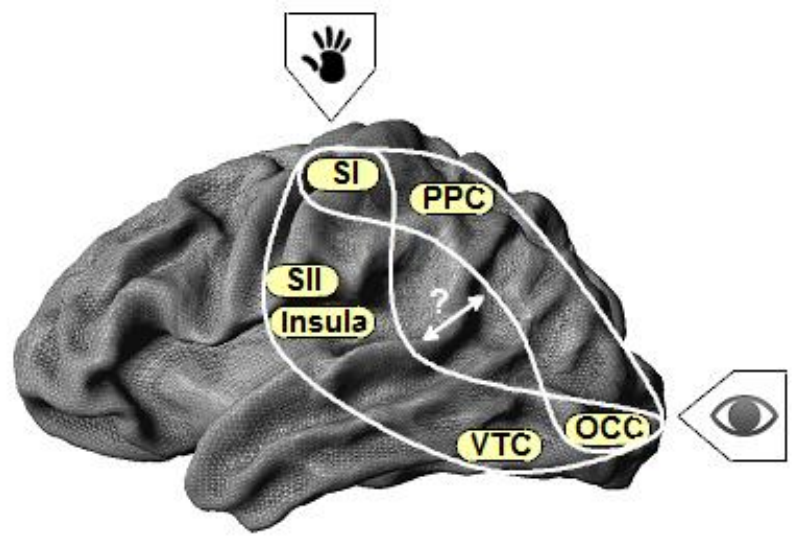

Figure 1. A working model of visual-haptic texture processing, segregated in a dorsal pathway for spatial density and a ventral pathway for roughness. SI = primary somatosensory cortex; $\mathrm{SII}=$ secondary somatosensory cortex; $\mathrm{PPC}=$ posterior parietal cortex; VTC $=$ ventral temporal cortex; OCC $=$ early occipital cortex. The reciprocal arrow represents the crosstalk between both pathways, to be specified in future research.

Although the proposed model focuses on texture processing related to identification and perception, the representation of spatial density in a dorsal cortical pathway is in line with the results of the study described in Chapter 4, showing that regions in the posterior parietal cortex represent differences in spatial density perceived by touch. This is also in accordance with earlier studies indicating a contribution of posterior parietal regions to tactile perception of spacing-related texture characteristics (Merabet et al., 2007; Zhang et al., 2005). The representation of visual and tactile spatial density information in a united cortical pathway is supported by the finding that the early visual and somatosensory cortex respond differently to matching than nonmatching visual-haptic dot pattern information (Chapter 3). Both direct and indirect cortico-cortical connections of the visual and somatosensory cortices would render such crossmodal interactions possible. In the macaque it was shown that the posterior parietal cortex receives projections from SI (Negyessy et al., 2006; Pons and Kaas, 1986) and projects in turn to early visual regions (Borra and Rockland, 2011). Functional connectivity studies, on the other hand, have reported a direct flow of somatosensory texture information from regions in the anterior parietal lobe to the posterior occipital cortex (Deshpande et al., 2008; Sathian et al., 2011). Even more interestingly, a recent study published by Liang and colleagues (2013) showed that early sensory cortices respond to stimuli that are not of their principal sensory modality. This finding is in line with the study described in Chapter 4, showing that regions in the posterior occipital cortex contain information about the perceived tactile spatial density of dot pattern textures even without concurrent visual information. There are two reasons why SII is not included in the cortical pathway suggested to mediate visual-haptic spatial density processing in the current working model. First, 
although neurophysiological studies on the monkey identified neurons in SI and SII that showed a discharge rate related to a change in texture, the majority of these neurons in SII were non-graded with spatial properties of the texture, while the opposite was true for texture-related neurons in SI (Jiang et al., 1997). Second, no neuroimaging study to date has reported a differential contribution of this region to spacing-related processing of textural stimuli. Hence, although it cannot be excluded that SII significantly contributes to the processing of textural spatial density, in light of the current body of literature it seems rather unlikely that its contribution is necessary to process differences in spatial density.

The cortical pathway mediating visual-haptic roughness perception in the proposed working model involves, in addition to the primary sensory cortices, the secondary somatosensory cortex, the insula and the ventral temporal cortex. As described in the introduction of this thesis, tactile roughness perception is mediated by both SA I and RA II (or PC) afferents (Gescheider and Wright, 2013; Hollins and Bensmaia, 2007), while spatial density of a pattern is likely only mediated by SA I afferents. Although both the primary and the secondary somatosensory cortex respond to vibrational and pressure information mediated by RA II (or PC) and SA I afferents (Chen et al., 2001; Francis et al., 2000; Friedman et al., 2004; Maldjian et al., 1999; Zhang et al., 2001), the response to vibrational information seems to be dominant in SII (Chung et al., 2013; Francis et al., 2000; Harrington and Hunter, 2001). These findings point to an involvement of SII in tactile roughness perception. Furthermore, several neuroimaging studies reported an involvement of the parietal operculum (including SII) and the insula in tactile roughness perception but failed to report any roughness-related responses in SI (Burton et al., 2008; Kitada et al., 2005; Simões-Franklin et al., 2011). The same pattern of results was also found in Chapter 4 of this thesis. Together, these results indicate that SI serves as an input area of somatosensory information, but might not contain explicit information discriminating between different levels of perceived roughness. The same might be true for visual roughness perception. While the primary visual cortex (pOCC) is the input region for visual stimulation, explicit visual roughness-related information might only be represented in higher-order cortices. One possible candidate for such a region is the ventral temporal cortex, as this area has been consistently reported to be involved in visual texture perception (Cant and Gooodale, 2007, 2011). Moreover, it was shown that the representation of material perception in the ventral visual pathway shifts gradually from a physical to a perceptual representation in ventral areas of the temporal cortex (Hiramatsu et al., 2011).

Interestingly, Chapter 4 also indicates an involvement of the VTC in tactile roughness perception. Previous studies focusing on the cortical processing of haptically perceived roughness failed to report such an involvement of visual areas, supporting to some extent the behavioral findings that roughness perception is predominantly haptic in nature. However, as shown 
in Chapter 2 haptic roughness experience can influence visual roughness estimation. Such an interaction of visual and haptic information can occur early in cortical processing or rather late. The activation of higher visual cortex in a purely tactile roughness estimation task, as presented in Chapter 4, indicates the latter. This is in line with the findings of Hiramatsu and colleagues (2011) who reported that a region around the fusiform gyrus and the collateral sulcus reflected not only visual (e.g. matte - glossy), but also non-visual material properties (e.g. smooth - rough), indicating its involvement in tactile information processing.

The somatosensory parietal part and the visual occipito-temporal part of the 'roughness' pathway are connected by the insula. In Chapter 4 differences in tactile roughness information were also conveyed by information in the operculo-insular cortex; a result that is in line with earlier findings (Kitada et al., 2005). The insula, with its connections to SI and SII and its projections to medial temporal lobe structures (Augustine et al., 1996; Mesulam et al., 1982; Mufson et al., 1982; Suzuki et al., 1994), represents an ideal candidate to connect the parietal and the occipito-temporal part of the pathway. Also functional connectivity studies showed that the posterior insula is involved in a functional network including somatosensory and ventral temporal cortex regions (Cauda et al., 2011). Hence, there are some indications in the literature that render such a parietal-occipitotemporal cortical pathway for visual-haptic roughness processing possible. However, since no study has investigated the cortical processing of visual roughness, the specific formation of that path and the interaction of the brain regions involved are highly speculative.

In summary, I proposed a working model characterized by a cortical segregation of visual-haptic texture processing in distinct pathways, a dorsal stream, involved in processing textural spatial density, and a ventral pathway, involved in textural roughness perception. Although at present this model is overly simplified, future studies should work towards its refinement and extension. In the next section, I will highlight questions that remain to be explored in future research.

\section{IMPLICATIONS AND FUTURE DIRECTIONS}

The model proposed above defines two segregated cortical pathways for visual-haptic information processing related to textural spatial density and textural roughness. The blanks in this model outweigh the known parts; some of the critical questions that need to be addressed will be discussed below.

Despite the existence of studies suggesting a ventral visual-haptic pathway for roughness perception, no neuroimaging study to date has investigated visual roughness perception in particular. Hence, in order to move beyond speculation to the level of empirical evidence, studies should be conducted to explore the brain regions involved in visual roughness perception. 
Furthermore, in order to specify a possible overlap of cortical pathways involved in visual and haptic texture processing, functional connectivity studies are required to investigate the cortical information flow in both sensory modalities. Instead of focusing on texture processing in general, investigating spatial density and roughness processing in isolation allows to derive useful information about direct or indirect interactions of brain regions forming the dorsal spatial density and ventral roughness pathway specified above. By combining visual and haptic information in a single condition, crossmodal influences in the cortical processing pathway of spatial density and roughness can be explored as well.

Another question that needs to be addressed by future research is the validity of the findings described in the present thesis for different sets of textural stimuli. In Chapter 2, visual roughness perception was shown to be influenced by previous haptic experience. Although this result is in line with previous studies showing that haptic information has a stronger effect than visual information on roughness perception, the generalizability of this finding should be tested by using different sets of unfamiliar textures. In general, all studies described in this thesis were conducted with the same stimulus set of unfamiliar dot pattern textures. Although this allowed a careful control of the physical stimulus characteristics, the ecological validity of such textures is limited. Hence, as a next step one should move from well-controlled artificial stimuli to more natural but also more complex textures.

\section{CONCLUDING REMARKS}

Texture perception has been studied since the early nineteen-eighties, but research on cortical processing of haptic and visual texture information has only emerged in recent years and is still in its infancy. The results of the studies reported in this thesis jointly suggest that visual and haptic texture information interact not only at the perceptual level but also at the cortical level. The findings were integrated in a cortical working model of visual-haptic texture processing. Future studies are required to specify the concrete points of interactions at the cortical level and the perceptual relevance for applications of virtual environments, just to mention one of many possible applications of this knowledge. Moreover, an integration of computational, physiological and neuroimaging models is needed to develop a fruitful exchange of knowledge in this research field and thus to generate integrative models, which will in turn broaden our understanding in the research field of texture perception. 


\section{REFERENCES}

Augustine, J.R., 1996. Circuitry and functional aspects of the insular lobe in primates including humans. Brain Res. Rev. 22, 229-244.

Borra, E., Rockland, K.S., 2011. Projections to early visual areas V1 and V2 in the calcarine fissure from parietal association areas in the macaque. Front. Neuroanat. 5, 1 - 12.

Burton, H., Sinclair, R.J., Wingert, J.R., Dierker, D.L., 2008. Multiple parietal operculum subdivisions in humans: Tactile activation maps. Somatosens. Mot. Res. 25, 149-162.

Cant, J.S., Goodale, M.A., 2011. Scratching beneath the surface: new insights into the functional properties of the lateral occipital area and parahippocampal place area. J. Neurosci. 31, 8248-8258.

Cant, J.S., Goodale, M.A., 2007. Attention to form or surface properties modulates different regions of human occipitotemporal cortex. Cereb. Cortex 17, 713731.

Cauda, F., D'Agata, F., Sacco, K., Duca, S., Geminiani, G., Vercelli, A., 2011. Functional connectivity of the insula in the resting brain. Neuroimage 55, 8-23.

Chen, L.M., Friedman, R.M., Ramsden, B.M., LaMotte, R.H., Roe, A.W., 2001. Finescale organization of SI (area $3 b$ ) in the squirrel monkey revealed with intrinsic optical imaging. J. Neurophysiol. 86, 3011-3029.

Chung, Y.G., Kim, J., Han, S.W., Kim, H.-S., Choi, M.H., Chung, S.-C., Park, J.-Y., Kim, S.-P., 2013. Frequency-dependent patterns of somatosensory cortical responses to vibrotactile stimulation in humans: A fMRI study. Brain Res. 1504, 47-57.

De Santis, L., Spierer, L., Clarke, S., Murray, M.M., 2007. Getting in touch: segregated somatosensory what and where pathways in humans revealed by electrical neuroimaging. Neuroimage 37, 890-903.

Dijkerman, H.C., de Haan, E.H.F., 2007. Somatosensory processes subserving perception and action. Behav. Brain Sci. 30, 189-239.

Deshpande, G., Hu, X., Stilla, R., Sathian, K., 2008. Effective connectivity during haptic perception: A study using Granger causality analysis of functional magnetic resonance imaging data. Neuroimage 40, 1807-1814.

Francis, S.T., Kelly, E.F., Bowtell, R., Dunseath, W.J.R., Folger, S.E., McGlone, F., 2000. fMRI of the responses to vibratory stimulation of digit tips. Neuroimage $11,188-202$.

Friedman, R.M., Chen, L.M., Roe, A.W., 2004. Modality maps within primate somatosensory cortex. Proc. Natl. Acad. Sci. U.S.A. 101, 12724-12729.

Gescheider, G.A., Wright, J.H., 2013. Roughness perception in tactile channels: Evidence for an opponent process in the sense of touch. Somatosens. Mot. Res. 30, 120-132.

Goodale, M.A., Milner, A.D., 1992. Separate visual pathways for perception and action. Trends Neurosci. 15, 20-25.

Harrington, G.S., Hunter Downs, J., 3rd, 2001. FMRI mapping of the somatosensory cortex with vibratory stimuli. Is there a dependency on stimulus frequency? Brain Res. 897, 188-192.

Heller, M.A., 1989. Texture perception in sighted and blind observers. Percept. Psychophys. 45, 49 - 54. 
Hiramatsu, C., Goda, N., Komatsu, H., 2011. Transformation from image-based to perceptual representation of materials along the human ventral visual pathway. Neuroimage 57, 482-494.

Hollins, M., Bensmaia, S.J., 2007. The coding of roughness. Can. J. Exp. Psychol. 61, 184-195.

Jiang, W., Tremblay, F., Chapman, C.E., 1997. Neuronal encoding of texture changes in the primary and the secondary somatosensory cortical areas of monkeys during passive texture discrimination. J. Neurophysiol. 77, 1656-1662.

Kitada, R., Hashimoto, T., Kochiyama, T., Kito, T., Okada, T., Matsumura, M., Lederman, S.J., Sadato, N., 2005. Tactile estimation of the roughness of gratings yields a graded response in the human brain: an fMRI study. Neuroimage 25, 90-100.

Kravitz, D.J., Saleem, K.S., Baker, C.I., Mishkin, M., 2011. A new neural framework for visuospatial processing. Nat. Rev. Neurosci. 12, 217-230.

Lederman, S.J., Abbott, S.G., 1981. Texture perception: Studies of intersensory organization using a discrepancy paradigm and visual versus tactual psychophysics. J. Exp. Psychol. Hum. Percept. Perform. 7, 902-915.

Lederman, S.J., Thorne, G., Jones, B., 1986. Perception of texture by vision and touch: Multidimensionality and intersensory integration. J. Exp. Psychol. Hum. Percept. Perform. 12, 169-180.

Liang, M., Mouraux, A., Hu, L., Iannetti, G.D., 2013. Primary sensory cortices contain distinguishable spatial patterns of activity for each sense. Nat. Commun. 4, 1979.

Maldjian, J.A., Gottschalk, A., Patel, R.S., Pincus, D., Detre, J.A., Alsop, D.C., 1999. Mapping of secondary somatosensory cortex activation induced by vibrational stimulation: an fMRI study. Brain Res. 824, 291-295.

Merabet, L.B., Swisher, J.D., McMains, S.A., Halko, M.A., Amedi, A., Pascual-Leone, A., Somers, D.C., 2007. Combined activation and deactivation of visual cortex during tactile sensory processing. J. Neurophysiol. 97, 1633-1641.

Mesulam, M.M., Mufson, E.J., 1982. Insula of the old world monkey. III: Efferent cortical output and comments on function. J. Comp. Neurol. 212, 38-52.

Mufson, E.J., Mesulam, M.M., 1982. Insula of the old world monkey. II: Afferent cortical input and comments on the claustrum. J. Comp. Neurol. 212, 23-37.

Negyessy, L., Nepusz, T., Kocsis, L., Bazso, F., 2006. Prediction of the main cortical areas and connections involved in the tactile function of the visual cortex by network analysis. Eur. J. Neurosci. 23, 1919-1930.

Pons, T.P., Kaas, J.H., 1986. Corticocortical connections of area 2 of somatosensory cortex in macaque monkeys: a correlative anatomical and electrophysiological study. J. Comp. Neurol. 248, 313-335.

Reed, C.L., Klatzky, R.L., Halgren, E., 2005. What vs. where in touch: an fMRI study. Neuroimage 25, 718-726.

Renier, L.A., Anurova, I., De Volder, A.G., Carlson, S., VanMeter, J., Rauschecker, J.P., 2009. Multisensory integration of sounds and vibrotactile stimuli in processing streams for "What" and "Where". J. Neurosci. 29, 10950-10960.

Sathian, K., Lacey, S., Stilla, R., Gibson, G.O., Deshpande, G., Hu, X., LaConte, S., Glielmi, C., 2011. Dual pathways for haptic and visual perception of spatial and texture information. Neuroimage 57, 462-475. 
Simões-Franklin, C., Whitaker, T.A., Newell, F.N., 2011. Active and passive touch differentially activate somatosensory cortex in texture perception. Hum. Brain Mapp. 32, 1067-1080.

Stilla, R., Sathian, K., 2008. Selective visuo-haptic processing of shape and texture. Hum. Brain Mapp. 29, 1123-1138.

Suzuki, W.A., Amaral, D.G., 1994. Perirhinal and parahippocampal cortices of the macaque monkey: cortical afferents. J. Comp. Neurol. 350, 497-533.

Van Boven, R.W., Ingeholm, J.E., Beauchamp, M.S., Bikle, P.C., Ungerleider, L.G., 2005. Tactile form and location processing in the human brain. Proc. Natl. Acad. Sci. U.S.A. 102, 12601-12605.

Zhang, M., Mariola, E., Stilla, R., Stoesz, M., Mao, H., Hu, X., Sathian, K., 2005. Tactile discrimination of grating orientation: fMRI activation patterns. Hum. Brain Mapp. 25, 370-377.

Zhang, H.Q., Murray, G.M., Coleman, G.T., Turman, A.B., Zhang, S.P., Rowe, M.J., 2001. Functional characteristics of the parallel SI- and SII-projecting neurons of the thalamic ventral posterior nucleus in the marmoset. J. Neurophysiol. 85, 1805-1822. 



\section{Acknowledgments}

I heard many people say that they first read the acknowledgments of a $\mathrm{PhD}$ thesis and then possibly read the rest. Now, if this holds for you too, please feel busted, go to the beginning of this book and read at least the first page of the introduction before returning to this part.

I know that this is a crucial, if not the most important part of the thesis because all the people that have a small or big share on this final product of four years of work will be finally acknowledged.

Let me start with the two people that hold the biggest share - my supervisors. Thank you Rainer for giving me the great opportunity to do a $\mathrm{PhD}$, thank you for supervising me, for always being positive and enthusiastic about new research ideas and for your trust in me. Amanda, you are probably the best daily supervisor a $\mathrm{PhD}$ student could wish for. You encouraged me to pursue my own ideas, but if needed you were there to extensively discuss these ideas, to fine-tune or dismiss them if necessary. Usually my emails to you would start with "I am sorry Amanda, but I underestimated the time it takes to ... the deadline is already tomorrow ... Could you nevertheless have a quick look at it and provide some feedback?" Amazingly, you always did provide very good and valuable feedback - thank you so much for all of this!

My great office mates, thank you Michael for your never-ending cheerfulness, Levin for your quiet support and Joost for patiently answering all my questions (although often starting with: "Ohhhh, that's easy!"). I do not know how you managed to endure my weekly Monday grumpiness and my uninterrupted soliloquies on the other weekdays, but you did. You provided the optimal working environment for me.

All present and former colleagues (or rather friends) of Brain Innovation, thank you! Thank you Armin and Fabrizio for all the help and the lively discussions. Fabrizio, you are the embodiment of Italian temperament in science. Armin, I really learned a lot about movies, although I am still an absolute beginner. Claudia, thank you for all the support, the nice company at the CODDE meetings and for providing new angles on my research topics. Bianca, thank you for always having kind words ready if needed. Hester, I never met a person that is more caring than you are, you are truly the soul of the company. Thank you for organizing all the social events and for being always 
helpful and encouraging. Pim: only this much, we miss the beer flag. Each Friday (after office hours), you helped us to have a smooth transition from work to weekend and this was required to start refreshed again on Monday. Maria, although you just started to work for the company right before I submitted my thesis, I am convinced that your very entertaining lessons on healthy living gave me all the vitamins required to finish the thesis. Julien, there is nothing more to say then we really miss your laughter and your cheerfulness.

Armin, Lars, Sven, Joost and Levin, I apologize for all my recurrent questions and I thank you for your great patience in handling them.

Jan and Henk, I really enjoyed our breaks outside, which provided in addition to the oxygen needed by my brain, also many entertaining theories and discussions.

Sanae, Luciana, Maria, Rita, Zoe, Anna G. and Anna Z., you girls are simply the best. Thank you for all the lunch breaks, the dinner/cooking evenings, the movie nights, the drinks after work.

All present and former colleagues at the $\mathrm{CN}$ department, you make the department such a great and inspiring place to work at. Thank you Eva, Gesa, Charlotte, Felix, Nina, Chris, Katie, Anke, Inge, Sven, Martin, Mehrdad, Sanne and Valentin. And what would the CN department be without Christl, Riny, and Annemie - I would have been completely lost without your help with all these organizational issues - a very very big thank you.

The CODDE network, next to the training and collaboration opportunities, we fellows also had a great time at the network meetings. Thanks Matt, Dev, Kurt, Cristiano, Dorita, Amar, Marco, Diderik, Michele, Andrea, Thomas, Vassilis, Saritha, Claudio, Yota, Dimitris. Thank you Andrew, Matthew and Zoe for organizing the CODDE network and for hosting me one month in Birmingham. I enjoyed my stay and learned a lot for the last $\mathrm{PhD}$ project.

I also would like to thank my friends that helped me to keep a very healthy work-life balance. Thank you Nicki, Andi, Janine, René, Tine, Markus, Uli, Berit, Susi und Jens for the fun weekends without research.

Finally yet most importantly, I want to acknowledge the big share that my family and Carsten hold on this $\mathrm{PhD}$ thesis. Without their unconditional support and their patience with me, this thesis would not be completed yet. Vielen Dank! 


\section{Curriculum Vitae}

Judith Eck was born on November $2^{\text {nd }}, 1982$ in Erfurt, Germany. In 2000 she graduated from the Washington Township High School in Valparaiso, USA. In Germany she graduated from high school at the Kooperative Gesamtschule am Schwemmbach in Erfurt in 2002. In the same year she enrolled at the Institute of Psychology at the Friedrich-Schiller-University in Jena, Germany. During her studies she worked from 2005 until 2006 as a student research assistant at the Faculty of Engineering in Building and Computer Science at the University of Applied Sciences, Erfurt. She completed her studies in Psychology successfully in 2008. In her Diploma thesis, under supervision of Prof. dr. Thomas Weiss, she studied the cortical processing of pain-related words in chronic pain patients using fMRI. For a related research project that she conducted during the same time together with Dr. Maria Richter, she received a research award for pain research from the German Society for the Study of Pain in 2010. After her graduation, she worked from 2008 until 2009 as a research assistant at the Otto-von-Guericke University in Magdeburg, Germany. In 2009, she moved to Maastricht and started to work as a $\mathrm{PhD}$ candidate under supervision of Prof. dr. Rainer Goebel and Dr. Amanda Kaas at Brain Innovation BV and the Department of Cognitive Neuroscience, Faculty of Psychology and Neuroscience, Maastricht University. Here she currently continues to work for Brain Innovation BV. 


\section{Publications}

Eck, J., Kaas, A. L., Goebel, R., 2013. Crossmodal interactions of haptic and visual texture information in early sensory cortex. Neuroimage 75, 123 135.

Eck, J., Kaas, A. L., Goebel R., 2011. The effect of sensory modality and previous experience on perceived roughness. Proceedings IEEE World Haptics Conference, 209-214.

Eck, J., Kaas, A. L., Mulders, J. L., Goebel, R., 2013. Roughness perception of unfamiliar dot pattern textures. Acta Psychol. (Amst), 143 (1), 20-34.

Eck, J., Kaas, A. L., Mulders, J. L., Hausfeld, L., Kourtzi, Z., Goebel, R., 2014. The effect of task instruction on haptic processing: The neural underpinning of roughness and spatial density perception. Manuscript submitted for publication.

Eck, J., Richter, M., Straube, T., Miltner, W.H.R., Weiss, T., 2011. Affective brain regions are activated during the processing of pain-related words in migraine patients. Pain, 152, 1104-1113.

Guerreiro M.J.S., Eck J., Moerel M., Evers L., van Gerven P., 2014. Attentional modulation in visual and auditory category-selective cortical areas. Manuscript submitted for publication.

Guerreiro M.J.S., Eck J., Moerel M., Evers L., van Gerven P., 2014. Attentional modulation of cortical activity in older age depends on sensory modality. Manuscript submitted for publication.

Richter, M., Eck, J., Straube, T., Miltner, W.H.R., Weiss, T, 2010. Do words hurt? Brain activation during the processing of pain-related words. Pain, 148, 198-205. 